\title{
Nonlinear rheology of colloidal dispersions
}

\author{
J M Brader \\ Fachbereich Physik, Universität Konstanz, D-78457 Konstanz, Germany
}

\begin{abstract}
Colloidal dispersions are commonly encountered in everyday life and represent an important class of complex fuid. Of particular signif cance for many commercial products and industrial processes is the ability to control and manipulate the macroscopic fow response of a dispersion by tuning the microscopic interactions between the constituents. An important step towards attaining this goal is the development of robust theoretical methods for predicting from f rst-principles the rheology and nonequilibrium microstructure of well def ned model systems subject to external fow. In this review we give an overview of some promising theoretical approaches and the phenomena they seek to describe, focusing, for simplicity, on systems for which the colloidal particles interact via strongly repulsive, spherically symmetric interactions. In presenting the various theories, we will consider frst low volume fraction systems, for which a number of exact results may be derived, before moving on to consider the intermediate and high volume fraction states which present both the most interesting physics and the most demanding technical challenges. In the high volume fraction regime particular emphasis will be given to the rheology of dynamically arrested states.
\end{abstract}

\section{Contents}

1. Introduction and overview

2. Continum mechanics approaches

2.1. The Lodge equation

2.2. The upper-convected Maxwell equation

2.3. Material objectivity

2.4. Beyond continuum mechanics

3. Microscopic dynamics

3.1. Non-interacting particles

3.2. Dimensionless parameters

3.3. Neglecting solvent hydrodynamics

3.4. Nonequilibrium states

4. Quiescent states

4.1. Hard-spheres

4.2. Auractive spheres

5. Rheological phenomenology

5.1. Zero-shear viscosity

5.2. Shear thimning

5.3. Shear thickening

5.4. Yicld stress

1 Present address: Department of Physics, University of Fribourg, CH-1700 Fribourg, Switzerland.
6. Theoretical approaches to fn id states 15

6.1. The pair Smoluchowski equation 15

6.2. Low yolume fraction 17

6.3. Intemediate volume fraction 19

6.4. Temporal locality versus memory functions 23

7. Glass theology 24

7.1. MCT inspired approaches 25

7.2. Integration through transients $\quad 26$

7.3. Translational invariance 27

7.4. Microscopic constitutive equation 27

7.5. Distorted structure factor 29

7.6. Applications 30

7.7. Schematic model 31

7.8. Yield stress surface $\quad 32$

8. Outlook 34

Acknowledgmenis $\quad 34$

References $\quad 34$

\section{Introduction and overview}

Complex fuids exhibit a rich variety of fow behaviour which depends sensitively upon the thermodynamic control parameters, details of the microscopic interparticle interactions 


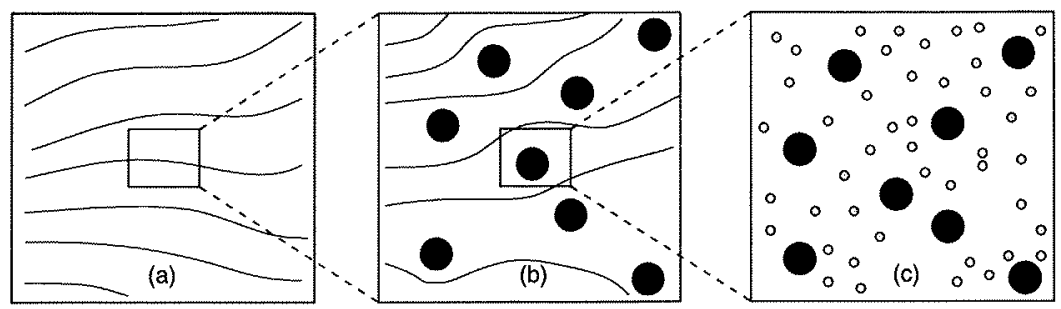

Figure 1. A schematic illustration of coarse graining as applied to colloidal dispersions. Continuum mechanics approaches treat the dispersion as a single continum flui (panel (a)), whereas a fully detailed picture is obtained by treating both colloids and solvent explicitly (panel (c)). The theoretical methods considered in this work operate at an intermediate level (panel (b)) in which the colloids are explicitly resolved but the solvent may be treated as a continuum.

and both the rate and specif $c$ geometry of the fow under consideration. The highly nonlinear response characteristic of complex fuids may be readily observed in a number of familiar household products [1]. For example, mayonnaise consists of a stabilized emulsion of oil droplets suspended in water and behaves as a soft solid when stored on the shelf but fows like a liquid, and is thus easy to spread, when subjected to shear fow with a knife [2]. This nonlinear viscoelastic fow behaviour, known as shear thinning, may be manipulated on the microscopic level by careful control of the oil droplet size distribution. In contrast, a dispersion of corn-starch particles in water, at suff ciently high concentrations, exhibits a dramatic increase in shear viscosity with increasing shear rate; a phenomenon called shear thickening $[3,4]$. Even the familiar practical problem of extracting tomato ketchup from a glass bottle presents a highly nonlinear $\mathrm{f}$ ow. In this case the applied shear stress, generally implemented by shaking, must exceed a critical value, the yield stress, before the ketchup begins to $\mathrm{fl} \mathrm{w}$ as desired.

Colloidal dispersions are a class of complex $\mathrm{fl}$ id which display all of the above mentioned nonlinear fow responses [5]. In addition to being of exceptional relevance for many technological processes, the considerable research interest in colloidal dispersions owes much to the existence of well characterized experimental systems for which the interparticle interactions may be tuned to relatively high precision (often possible by simply varying the solvent conditions) [6]. The ability to control the microscopic details of the colloidal interaction facilitates comparison of experimental results with theoretical calculations and computer simulations based on idealized models (see e.g. [7-9]). In particular, the size of colloidal particles makes possible light scattering, neutron scattering and microscopy experiments which provide information inaccessible to experiments on atomic systems and which have enabled various aspects of liquid state theory to be tested in detail ${ }^{2}$.

The typical size of a colloidal particle lies in the range $10 \mathrm{~nm}-1 \mu \mathrm{m}$ and thus enables a fairly clear separation of

\footnotetext{
2 For example, in [8] and [9] dynamic light scattering was employed to measure the coherent transient density correlator of spherical hard-sphere like coltoids. The data conf rmed the factorization properties of the $a$ and $\beta$ relaxation, as predicted by the mode-coupling theory of the glas transition $[10,11]$. In $[12]$ confocal microscopy experiments on a mixture of PMMA colloids and non-adsorbing polymer were used to conf $\mathrm{rm}$ the capillary wave theory of the f uctuating interface between demixed f uid phases [13].
}

length- and timescales to be made between the colloids and the molecules of the solvent in which they are dispersed. As a result, a reasonable frst approximation is to represent the solvent as a continuum fuid, generally taken to be Newtonian and thus characterized by a constant solvent viscosity (see fgure 1). For suspended particles with a length-scale greater than approximately $1 \mu \mathrm{m}$ the continuum approximation of the solvent is completely appropriate. However, this becomes questionable as the average size of the particles is reduced below a few nanometres, at which point the discrete nature of the solvent can no longer be ignored. Colloidal particles occupy an intermediate range of length-scales for which a continuum approximation for the solvent must be supplemented by the addition of $\mathrm{frst}$ order Gaussian fuctuations (Brownian motion) about the average hydrodynamic $f$ elds describing the viscous fow of the continuum solvent.

The Brownian motion resulting from solvent fuctuations not only plays an important role in determining the microscopic dynamics; it is essential for the existence of a unique equilibrium microstructure. With the important exception of arrested glasses and gels, the presence of a stochastic element to the particle motion allows a full exploration of the available phase space and thus enables application of Boltzmann-Gibbs statistical mechanics to quiescent (and ergodic) colloidal dispersions. While the specifi nature of the balance between Brownian motion, hydrodynamic and potential interactions depends upon both the observable under consideration and the range of system parameters under investigation, it is the simultaneous occurrence of these competing physical mechanisms which gives rise to the rich and varied rheological behaviour of dispersions. Unfortunately, the complicated microscopic dynamics presented by dispersions also serves to complicate the theoretical description of these systems [14].

The present review has been written with a number of aims in mind. On one hand, we would like to present a relatively concise overview of the main phenomenological features of the rheology of dispersions of spherical colloidal particles. In order to reduce the parameter space of the discussion, emphasis will be placed on the simple hard-sphere model for which the space of control parameters is restricted to two dimensions (volume fraction and fow rate). While both attractive colloids and the response to non-shear fows will be 
addressed, no attempt has been made to be comprehensive in this respect. Another primary aim of the present work is to provide an overview, within the context of the aforementioned phenomenology, of microscopically motivated approaches to the rheology and fow induced microstructure of colloidal dispersions. Although we will discuss some less well founded 'schematic model' approaches, the focus here is upon 'f rstprinciples' theories which prescribe a route to go from a well def ned microscopic dynamics to closed expressions for macroscopically measurable quantities.

The formulation of a robust theory of dispersion rheology from microscopic starting points constitutes a formidable problem in nonequilibrium statistical mechanics. Although considerable progress has been made in this direction, a comprehensive constitutive theory analogous to that of Doi and Edwards for entangled linear polymers [21-24] remains to be found. At present there exist a number of alternative microscopic theoretical approaches to dispersion rheology which, despite showing admirable success within limited ranges of the system parameters, have so far been unable to provide a unifed global picture of the microscopic mechanisms underlying the rheology of colloidal dispersions. Despite common starting points (the many-body Smoluchowski equation) the disparate nature of the subsequent approximations, each tailored to capture a particular physical aspect of the cooperative particle motion, make it diff cult to establish clear relations between different theoretical approaches. A goal of this work is thus to clarify the range of validity of the various theoretical approaches and to identify common ground. We note that the present work is well complemented by a number of recent reviews addressing dispersion rheology from both experimental $[25,26]$ and theoretical perspectives $[19,27,28]$.

The paper is organized as follows: in section 2 we will discuss briefy some traditional continuum mechanics approaches to rheology, both to give a feeling for the spirit of such work and to put into context some of the microscopic results presented later (in section 7). In section 3 we will introduce and discuss in some detail the Smoluchowski equation def ning the microscopic dynamics under consideration. In section $\&$ we will consider the equilibrium and nonequilibrium phase behaviour of hardsphere colloids in the absence of fow, which is a necessary pre-requisite to the subsequent discussions. In section 5 we will give a brief overview of the relevant basic phenomenology of dispersion rheology, including the shear thinning and shear thickening of colloidal fuids and the yielding of colloidal glasses. In section 6 we will consider the various theoretical approaches to treating colloidal fuids under external fow. In particular, exact results for the microstructure and rheology of low volume fraction systems and their (approximate) extension to f nite fuid volume fractions are discussed in sections 6.2 and 6.3 , respectively. In section 7 , we consider the recently developed mode-coupling based approaches to the rheology of dense colloidal suspensions which enable glass rheology to be addressed. Finally, in section 8 we will provide an outlook for future work and identify possible new avenues for theoretical investigation.

\section{Continuum mechanics approaches}

Rheology is primarily an experimental discipline. Indeed, one of the simplest experiments imaginable is to exert a force on a material in order to see how it deforms in response. More precisely, in a controlled rheological experiment one measures either the stress arising from a given strain or, more commonly, the strain accumulated following application of an applied stress. In practice, both stress controlled and strain controlled experiments are performed and provide complementary information regarding the response of a material sample. For the purpose of this review we will focus upon situations in which a homogeneous strain $\mathrm{feld}$ is prescribed from the outset. The description of experiments for which macroscopic stress is employed as a control parameter poses an enormous challenge for microscopically based theories and demands careful consideration of the nontrivial mechanisms by which the applied stress propagates into the sample from the boundaries.

Given the apparent complexity of any microscopic theory, it is quite natural to begin $\mathrm{frst}$ at a more coarse-grained level of description in an effort to establish the general phenomenology and mathematical structure of the governing equations at the continuum level. Historically, this methodology was pioneered by Maxwell in his 1863 work on viscoelasticity and continued to develop into the following century through the efforts of distinguished rheologists such as Rivlin and Oldroyd [29]. While much of this early work aimed to achieve a more fundamental mathematical understanding of viscoelastic response, strong additional motivation was provided by experiments on polymeric systems which exposed a large variety of interesting nonlinear rheological phenomena in need of theoretical explanation. Theoretical approaches to continuum rheology thus seek to obtain a constitutive equation relating the stress, a tensorial quantity describing the forces acting on the system [30], to the deformation history encoded in the strain tensor.

The typical 'rational mechanics' approach to this problem is to assume a suff ciently general integral or differential constitutive relation between stress and strain and to then constrain this as much as possible via the imposition of certain exact or approximate macroscopic symmetry, conservation and invariance principles $[22,29,31]$. The clear drawback to this methodology is that the entire particulate system is viewed as a single continuum feld, thus losing any contact to the underlying colloidal interactions and microstructure ultimately responsible for the macroscopic response (see fgure 1). As a result, such constitutive theories are neither material specifi nor genuinely predictive in character. Despite these shortcomings, the continuum mechanics approach to rheology has attained a great level of ref nement and can be applied to $f t$ experimental data from a wide range of physical systems [22, 29]. Moreover, the experience gained through continuum mechanics modelling may well prove useful in guiding the construction of more sophisticated microscopic theories by providing constraints on the admissible mathematical form of the constitutive equations. 


\subsection{The Lodge equation}

It is perhaps instructive to give an illustration of the spirit in which phenomenological constitutive relations may be constructed using continuum mechanics concepts. The example we choose is not only of intrinsic interest, but will also prove relevant to the discussion of a recent microscopically based theory of glass rheology [16-18] to be discussed in section 7 . We consider a viscoelastic fl id subject to shear deformation with fow in the $x$-direction and shear gradient in the $y$-direction (a convention we will continue to employ throughout the present work). Suppose that we wish to determine the inf nitesimal shear stress $\mathrm{d} \sigma_{x y}$ at time $t$ arising from a small strain increment $\mathrm{d} \gamma$ at an earlier time $t^{\prime}$. As the material is viscoelastic, it is reasonable to assume that the inf uence of the strain increment $\mathrm{d} \gamma\left(t^{\prime}\right)=\dot{\gamma}\left(t^{\prime}\right) \mathrm{d} t^{\prime}$ on the stress at time $t$ must be weighted by a decaying function of the intervening time $t-t^{\prime}$, in order to represent the infl ence of dissipative processes. Adopting a simple exponential form for the relaxation function it is thus intuitive to write

$$
\mathrm{d} \sigma_{x y}(t)=G_{\infty} \exp \left[\begin{array}{c}
t-t^{\prime} \\
\tau
\end{array}\right] \dot{\gamma}\left(t^{\prime}\right) \mathrm{d} t^{\prime},
$$

where $\tau$ is a relaxation time and $G_{\infty}$ is an elastic constant (the inf nite frequency shear modulus). Assuming linearity, the total stress at time $t$ may thus be constructed by summing up all of the inf nitesimal contributions over the entire fow history, which we take to extend into the inf nite past. We thus arrive at

$$
\sigma_{x y}(t)=\int_{-\infty}^{t} \mathrm{~d} t^{\prime} G_{\infty} \exp \left[\begin{array}{c}
t-t^{\prime} \\
\tau
\end{array}\right] \dot{\gamma}\left(t^{\prime}\right) .
$$

Partial integration leads fnally to

$$
\sigma_{x y}(t)=\frac{1}{\tau} \int_{-\infty}^{t} \mathrm{~d} t^{\prime} G\left(t-t^{\prime}\right) \gamma\left(t, t^{\prime}\right)
$$

where $G(t)=G_{\infty} \exp [-t / \tau]$ is the shear modulus and $\gamma\left(t, t^{\prime}\right)$ is the accumulated strain $\gamma\left(t, t^{\prime}\right)=\int_{t^{\prime}}^{t} \mathrm{~d} s \dot{\gamma}(s)$. The simple integral relation ( 3 ) between shear stress and shear strain was f rst considered by Boltzmann. Indeed, the assumption that the stress increments (1) may be summed linearly to obtain the total stress is often referred to as the 'Boltzmann superposition principle'.

In order to extend (3) to a tensorial relation, i.e. a true constitutive equation, an appropriate tensorial generalization of the accumulated strain $\gamma\left(t, t^{\prime}\right)$ must be identif ed. For the spatially homogeneous deformations under consideration the translationally invariant deformation gradient tensor $\boldsymbol{E}\left(t, t^{\prime}\right)$ transforms a vector ('material line') at time $t$ ' to a new vector at later time $t$ via $\mathbf{r}(t)=E\left(t, t^{\prime}\right) \cdot \mathbf{r}\left(t^{\prime}\right)$, where $E_{\alpha \beta}=$ $\partial r_{\alpha} / \partial r_{\beta}$. An alternative nonlinear choice of strain measure is the symmetric Finger tensor $\boldsymbol{B}\left(t, t^{\prime}\right)=\boldsymbol{E}\left(t, t^{\prime}\right) \boldsymbol{E}^{\mathrm{T}}\left(t, t^{\prime}\right)$. The Finger tensor contains information about the stretching of material lines during a deformation but is invariant with respect to solid body rotations of the material sample. For simple shear the Finger tensor is given explicitly by

$$
\boldsymbol{B}=\left(\begin{array}{ccc}
1+\gamma^{2} & \gamma & 0 \\
\gamma & 1 & 0 \\
0 & 0 & 1
\end{array}\right)
$$

where $\gamma \equiv \gamma\left(t, t^{\prime}\right)$. The accumulated strain in the integrand of equation (3) can thus be identif ed as the $x y$ element of $B\left(t, t^{\prime}\right)$. This suggests that the Boltzmann integral form (3) may be extended using the simple ansatz

$$
\sigma(t)=\int_{-\infty}^{t} \mathrm{~d} t^{\prime} \boldsymbol{B}\left(t, t^{\prime}\right) G_{\infty} \mathrm{e}^{-\left(t-t^{\prime}\right) / \tau},
$$

for the full stress tensor (see section 2.3 below for more justif cation of this nontrivial step). Equation (5) is known as the Lodge equation in the rheological literature and is applicable in both the linear and nonlinear viscoelastic regime [22].

\subsection{The upper-convected Maxwell equation}

The assumption of an exponentially decaying shear modulus is generally attributed to Maxwell, who realized that this choice enabled an interpolation between a purely elastic response to deformations rapid on the timescale set by $\tau$ and a viscous, dissipative response in the limit of slowly varying strain $f$ elds. In fact, the Lodge equation derived above is simply the integral form of a nonlinear (differential) Maxwell equation. In order to show this we frst differentiate (5) to obtain

$$
D \boldsymbol{D} \boldsymbol{D t}+{ }_{\tau}^{1} \sigma={ }_{\tau}^{G_{\infty}} \mathbf{1}
$$

where we have introduced the upper-convected derivative [29]

$$
\begin{gathered}
D \sigma \\
D t
\end{gathered}=\dot{\sigma}(t)-\kappa(t) \sigma(t)-\sigma(t) \kappa^{\mathrm{T}}(t)
$$

and where the velocity gradient tensor $\kappa(t)$ is def ned in terms of the deformation gradient tensor via

$$
{ }_{\partial t}^{\partial} E\left(t, t^{\prime}\right)=\kappa(t) E\left(t, t^{\prime}\right) .
$$

For an incompressible material the stress is only determined up to a constant isotropic term. Equation (6) may thus be expressed in an alternative form by frst def ning a new stress tensor

$$
\Sigma=\sigma-G_{\infty} \mathbf{1}
$$

and substituting for $\sigma$ in equation (6). This yields

$$
\frac{D \Sigma}{D t}+{ }_{\tau}^{1} \Sigma=G_{\infty}\left(\kappa(t)+\kappa^{\top}(t)\right)
$$

This differential form of the Lodge equation is known as the upper-convected Maxwell equation [22] and is a nonlinear generalization of Maxwell's original scalar model to the full deviatoric stress tensor. Historically, the upper-convected Maxwell equation was $\mathrm{f}$ rst proposed by Oldroyd [29] directly on the basis of Maxwell's differential form.

\subsection{Material objectivity}

The assumption that one can go from (3) to (5) on the basis of a single off-diagonal element appears at $f$ rst glance to be rather ad hoc. On one hand, this choice can be justif ed retrospectively, using the fact that the Lodge equation (5) is 
derivable from a number of simple molecular models, e.g. the dumbbell model for dilute polymer solutions [22]. However, from a continuum mechanics perspective (5) is the simplest generalization of (3) which satisf es the "principle of material objectivity'. This principle expresses the requirement that the constitutive relationship between stress and strain tensors should be invariant with respect to rotation of either the material body or the observer, thus preventing an unphysical dependence of the stress on the state of rotation. That this symmetry is an approximation becomes clear when considering the material from a microscopic viewpoint: in a noninertial rotating frame the apparent forces clearly lead to particle trajectories which depend upon the angular velocity. For many systems the neglect of these effects on the macroscopic response of the system is an extremely good approximation. For the overdamped colloidal dynamics considered in this work inertia plays no role and the principle of material objectivity is exact ${ }^{3}$.

Mathematically, it is straightforward to check whether or not a proposed tensorial constitutive equation is material objective. When subject to a time-dependent rotation $\boldsymbol{R}(t)$ the deformation gradient tensor transforms as

$$
\hat{\boldsymbol{E}}\left(t, t^{\prime}\right)=\boldsymbol{R}(t) \boldsymbol{E}\left(t, t^{\prime}\right) \boldsymbol{R}^{\mathrm{T}}\left(t^{\prime}\right),
$$

where $\hat{E}$ is the deformation gradient in the rotating frame. The dependence of $\hat{\boldsymbol{E}}$ upon the state of rotation arises because $\boldsymbol{E}$ contains information about both the stretching and rotation of material lines. Insertion of the transformed tensor (11) into the constitutive equation for the stress thus corresponds to a rotation of the material sample. Material objectivity is verif ed if the resulting stress tensor is given by

$$
\hat{\boldsymbol{\sigma}}(t)=\boldsymbol{R}(t) \sigma(t) \boldsymbol{R}^{\mathrm{T}}(t) .
$$

As noted, the Finger tensor $\boldsymbol{B}$ contains only information about the stretching of material lines and transforms under rotation according to

$$
\hat{B}\left(t, t^{\prime}\right)=R(t) B\left(t, t^{\prime}\right) R^{\top}(t) .
$$

The material objectivity of the Lodge equation (5), and thus the upper-convected Maxwell equation (10), follows trivially from the fact that $\sigma$ is a linear functional of $B$. Many phenomenological rheological models thus start by assuming a general functional dependence $\sigma(t)=\mathcal{F}[\boldsymbol{B}]$ in order to guarantee a rotationally invariant theory.

The vast majority of microscopically motivated theories of dispersion rheology treat only a single scalar element of the stress tensor (generally the shear stress $\sigma_{x y}$ ). Indeed, the rarity of microscopic tensorial constitutive theories may well be the primary reason for the apparent gap between continuum and statistical mechanical theories aiming to describe common phenomena. We will revisit the concept of material objectivity in section 7 when considering a recently proposed tensorial constitutive equation for dense dispersions.

3 A useful discussion of material objectivity may be found in [33]. In Addition, we refer the reader to [32], which documents the insightful comments of de Gennes regarding this issue.

\subsection{Beyond continuum mechanics}

In the last decade, significa $t$ progress has been made in understanding the response of colloidal dispersions to external fow on a level which goes beyond the fully coarse-grained phenomenological approaches of traditional continuum rheology. Important steps towards a more ref ned picture have been provided by studies based on mesoscopic models $[25,34-36]$. However, while such phenomenological approaches can reveal generic features of the rheological response, they are not material specif $c$ and can therefore address neither the inf uence of the microscopic interactions on the macroscopic rheology nor the underlying microstructure, as encoded in the particle correlation functions. This deeper level of insight is provided by fully microscopic approaches which start from a well def ned particle dynamics and, via a sequence of either exact or clearly specifie approximate steps, lead to closed expressions for macroscopically measurable quantities. The symmetry, invariance and conservation principles used as input in the construction of continuum theories, such as the material objectivity discussed in section 2.3, should then emerge directly as a consequence of the microscopic interactions. Such an undertaking clearly requires the machinery of statistical mechanics.

Theories founded in statistical mechanics provide information regarding the correlated motion of the constituent particles and are thus capable, at least in principle, of capturing nontrivial and potentially unexpected cooperative behaviour as exhibited by equilibrium and nonequilibrium phase transitions. This ability to capture emergent phenomena is in clear contrast to continuum approaches where such physical mechanisms must be input by hand. An additional advantage of a statistical mechanics based approach to rheology over the direct application of continuum mechanics is that important additional information is provided regarding the microstructure of the system, as encoded in the correlation functions. It thus becomes possible to connect the constitutive relations to the underlying correlations between the colloidal particles and obtain microscopic insight into the macroscopic rheological response. Additional motivation to theoretically 'look inside' the fowing system is provided by developments in the direct visualization and tracking of particle motion in experiments on colloidal dispersions (confocal microscopy) [37-39], together with advances in the computer simulation of model systems under fow [40-42].

Although beyond the scope of the present work, we note that the inf uence of steady shear fl $\mathrm{w}$ on glassy states has been addressed, albeit in an abstract setting, by generalized meanfeld theories of spin glasses $[43,44]$. Spin glass approaches have proved useful in describing the dynamical behaviour of quiescent systems [45]. In order to mimic the effect of shear fow a nonconservative force is introduced to bias the dynamics and break the condition of detailed balance characterizing the equilibrium state [46]. While the abstract nature of these treatments certainly lends them a powerful generality, the lack of material specific ty makes diffic it a direct connection to experiment. 


\section{Microscopic dynamics}

Before addressing the phenomenology (section 5) and approximate theories (sections 6 and 7) of colloid rheology it is rewarding to $\mathrm{f}$ rst consider in detail the microscopic equationof-motion determining the overdamped colloidal dynamics. By a careful assessment of the fundamental equation-of-motion a number of general observations and comments can be made regarding the character of nonequilibrium states, solutions in special limits, important dimensionless parameters and inf uence of hydrodynamics, which are independent of the specif c system or approximation scheme under consideration.

We consider a system consisting of $N$ Brownian colloidal particles interacting via spherically symmetric pairwise additive interactions and homogeneously dispersed in an incompressible Newtonian fuid of given viscosity. The probability distribution of the $N$-particle conf guration is denoted by $\Psi(t)$ and satisf es the Smoluchowski equation [14]

$$
\frac{\partial \Psi(t)}{\partial t}+\sum_{i} \boldsymbol{d}_{i} \cdot \mathbf{j}_{i}=0
$$

where the probability fux of particle $i$ is given by

$$
\mathbf{j}_{i}=\mathbf{v}_{i}(t) \Psi(t)-\sum_{j} D_{i j} \cdot\left(\partial_{j}-\beta \mathbf{F}_{j}\right) \Psi(t),
$$

where $\beta=1 / k_{\mathrm{B}} T$ is the inverse temperature. The hydrodynamic velocity of particle $i$ due to the applied fow is denoted by $\mathbf{v}_{i}(t)$ and the diffusion tensor $\boldsymbol{D}_{i j}$ describes (via the mobility tensor $\Gamma_{i j}=\beta D_{i j}$ ) the hydrodynamic mobility of particle $i$ resulting from a force on particle $j$. The hydrodynamic velocity can be decomposed into aff ne and particle induced fuctuation terms $\mathbf{v}_{i}(t)=\kappa(t) \cdot \mathbf{r}_{i}+\mathbf{v}_{i}^{\mathbf{f}}(t)$, where $\mathbf{v}_{i}^{\mathbf{f}}(t)$ can be expressed in terms of the third rank hydrodynamic resistance tensor [47]. The force $\mathbf{F}_{j}$ on particle $j$ is generated from the total potential energy according to $\mathbf{F}_{j}=-\partial_{j} U_{\mathrm{N}}$, where, in the absence of external f elds, $U_{\mathrm{N}}$ depends solely on the relative particle positions. The three terms contributing to the fux thus represent the competing effects of (from left to right in (15)) external $\mathrm{fl} \mathrm{w}$, diffusion and interparticle interactions.

While the Smoluchowski equation (14) is widely accepted as an appropriate starting point for the treatment of colloidal dynamics, alternative approaches based on the FokkerPlanck equation have also been investigated [48]. On the Fokker-Planck level of description the distribution function retains a dependence on the particle momenta. Although this makes possible the treatment of systems with a temperature gradient (leading to thermophoretic effects), considerable complications arise when attempting to treat hydrodynamic interactions which make preferable the Smoluchowski equation.

For the special case of monodisperse hard-spheres at fnite volume fractions under steady fow equation (14) can be numerically integrated over the entire fuid range using computationally intensive Stokesian dynamics simulation $[41,49,50]$. This simulation technique includes the full solvent hydrodynamics and provides a useful benchmark

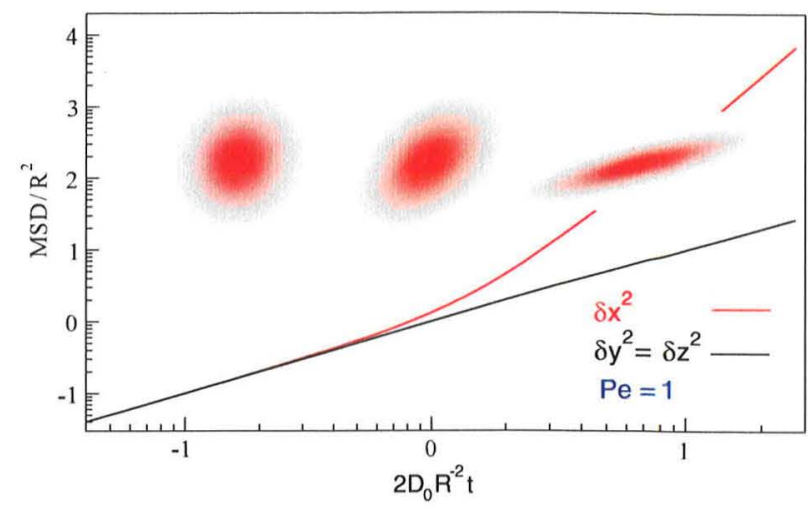

Figure 2. The mean-squared-displacement of non-interacting colloidal particles in f ow $(x)$, gradient $(y)$ and vorticity $(z)$ directions as a function of time. The MSD in fow direction exhibits enhanced diffusion ('Taylor dispersion') for values of the shear strain greater than unity. Also shown are contour plots of the (non-normalized) probability distribution $P_{1}(\mathbf{r}, t) N(t)$ (see equation (16)) in the $z=0$ plane at times $2 D_{0} R^{-2} t=0.15,1$ and 5 , demonstrating shear induced anisotropy for $\gamma>1$ related to the onset of Taylor dispersion.

for theoretical approaches (for an overview of the computer simulation of viscous dispersions we refer the reader to [51] and references therein). While Stokesian dynamics simulations have focused primarily on simple shear, results have also been reported for extensional fow geometries [52].

\subsection{Non-interacting particles}

For the special case of non-interacting particles $\left(\mathbf{F}_{j}=\right.$ 0 ) equations (14) and (15) describe the conf gurational probability distribution of an ideal gas under externally applied fow and may be solved analytically using the method of characteristics [53]. For non-interacting particles under steady shear the many-particle distribution function is given by a product of single particle functions $\Psi\left(\left\{\mathbf{r}_{i}\right\}, t\right)=P_{1}\left(\mathbf{r}_{1}, t\right) \times$ $\cdots \times P_{1}\left(\mathbf{r}_{\mathrm{N}}, t\right)$, where $P_{1}$ is given by

$$
P_{1}(\mathbf{r}, t)=\frac{1}{N(t)} \exp \left[\begin{array}{cc}
-x^{2}-y^{2}\left(1+\gamma_{3}^{2}\right)+\gamma x y & z^{2} \\
N(t) & 4 D_{0} t
\end{array}\right]
$$

when the initial condition $P_{1}(\mathbf{r}, 0)=\delta(0)$ is employed. The normalization is given by $N(t)=\left(4 \pi D_{0} t\right)^{3}\left(1+(\dot{\gamma} t)^{2} / 12\right)$ and the strain by $\gamma=\dot{\gamma} t$. Given a suitably localized initial density distribution equation (16) essentially describes the dispersion of a colloidal droplet in a solvent (e.g. ink in water) under shear, as is apparent from f gure 2, which shows contour plots of the probability distribution at three different times for a given shear rate.

Although non-interacting colloids represent a trivial case, it is nevertheless instructive to consider the mean-squareddisplacement (MSD), characterizing the diffusive particle motion, both parallel and orthogonal to the fow direction in simple shear [54]. In both the vorticity and shear gradient directions, fow has no inf uence and the equilibrium result is recovered, $\delta z^{2}=\delta y^{2}=2 D_{0} t$, with $D_{0}$ the single particle diffusion coeff cient. In the fow direction the MSD is 
enhanced by a coupling between Brownian motion and aff ne advection, yielding $\delta x^{2}=2 D_{0} t\left(1+\dot{\gamma}^{2} t^{2} / 3\right)$, where $\dot{\gamma}$ is the shear rate. The physical origin of this enhanced diffusion, termed 'Taylor dispersion' [55], is that the random motion of a given colloid leads to its displacement into planes of laminar fow with a velocity different from that of the original point. This constant and random 'changing of lanes' leads, on the average, to a dramatically increased rate of diffusion in the direction of fow. The accelerated rate of mixing achieved by stirring a dilute dispersion is thus almost entirely attributable to local Taylor dispersion. We note also that analogous effects arising from fow-diffusion coupling can also be identif ed in other fow geometries, such as the practically relevant case of Poiseuille fow along a cylindrical tube [54].

\subsection{Dimensionless parameters}

The Smoluchowski equation describes the dynamics of spherical colloidal particles dispersed in an incompressible Newtonian fuid and provides the fundamental starting point for all theoretical work to be described in the following sections. An appropriate dimensionless Reynolds number governing the solvent fow may be def ned as $R e=\rho \dot{\gamma} R^{2} / \eta$, with $\dot{\gamma}$ a characteristic fl $\mathrm{w}$ rate, $\rho$ is the density, $\eta$ the solvent viscosity and $R$ the colloidal length-scale. Due to the small size of the colloidal particles $R e$ remains small for all situations of physical relevance and the Stokes equations, rather than the more complicated Navier-Stokes equations, may thus be employed in treating the solvent $\mathrm{f}$ ow.

Given that $R e$ remains small, two dimensionless parameters are of particular importance in determining the equilibrium and nonequilibrium behaviour. The frst of these is the colloidal volume fraction $\phi=4 \pi n R^{3} / 3$, with number density $n$ and particle radius $R$. The maximum volume fraction achievable for monodisperse spheres is 0.74 corresponding to an optimally packed face-centred-cubic crystal structure. For the purposes of the present work we will $f$ nd it convenient to divide the physical range of volume fractions into three subregions: (i) low packing, $\phi<0.1$, (ii) intermediate packing, $0.1<\phi<0.494$, and (iii) high packing, $0.494<\phi$. While this division is somewhat arbitrary, it will later prove useful in discussing the various theoretical approximation schemes currently available.

The second important dimensionless parameter is the Peclet number $P e=\dot{\gamma} R^{2} / 2 D_{0}$ [14]. The Peclet number is a measure of the importance of advection relative to Brownian motion and determines the extent to which the microstructure is distorted away from equilibrium by the fow $\mathrm{f}$ eld. In the limit $P e \rightarrow 0$ Brownian motion dominates and the thermodynamic equilibrium state is recovered. Conversely, in the strong fow limit, $P e \rightarrow \infty$, solvent mediated hydrodynamic interactions may be expected to dominate the particle dynamics, although, in practice, surface roughness and other perturbing effects turn out to complicate this limit [56] (see section 6.2 for more details on this point).

Finally, we would like to note that there exists a further, nontrivial dimensionless quantity implicit in the many-body Smoluchowski equation (14). An increase in either the dispersion volume fraction or attractive coupling between particles is accompanied by an increase in the structural relaxation timescale of the system $\tau_{\alpha}$ characterizing the temporal decay of certain two-point autocorrelation functions. This enables the Weissenberg number to be def ned as $W i=\dot{\gamma} \tau_{\alpha}$. For intermediate and high volume fractions, particularly those close to the colloidal glass transition, it is the Weissenberg number, rather than the 'bare' Peclet number $P e$, which dominates certain aspects of the nonlinear rheological response, as has been emphasized in [57]. For the low volume fraction systems to be considered in section 6.2 the structural relaxation timescale is set by $R^{2} / 2 D_{0}$, leading to $P e=W i$.

\subsection{Neglecting solvent hydrodynamics}

In many approximate theories aiming to describe intermediate and high volume fraction dispersions the inf uence of solvent hydrodynamics beyond trivial advection is neglected from the outset. For certain situations (e.g. glasses) this approximation is partially motivated by physical intuition, however, in most cases, the omission of solvent hydrodynamics is an undesirable but unavoidable compromise made in order to achieve tractable closed expressions. Accordingly, the expression for the probability fux (15) is approximated in two places, which we will now discuss in turn.

The frst approximation is to set $D_{i j}=D_{0} \delta_{i j}$, thus neglecting the inf uence of the conf guration of the $N$ colloidal particles on the mobility of a given particle. For low and intermediate volume fraction $f$ uids this may be reasonable for $P e \ll 1$ but can be expected to break down for $P e>1$ as hydrodynamics becomes increasingly important in determining the particle trajectories. In particular, the near-fie d lubrication forces [47] which reduce the mobility when the surfaces of two particles approach contact play an important role in strong fow and are responsible for driving cluster formation and shear thickening [58] (see section 5.3). For dense colloidal suspensions close to a glass transition the role of hydrodynamics is less clear. For certain situations of interest (e.g. glasses close to yield) the relevant value of $P e$ is very small and suggests that hydrodynamic couplings should not be of primary importance.

The second common approximation to (15) arising from the neglect of solvent hydrodynamics is the assumption of a translationally invariant linear fow prof le $\mathbf{v}(\mathbf{r}, t)=\kappa(t) \cdot \mathbf{r}$, where $\kappa(t)$ is the (traceless) time-dependent velocity gradient tensor introduced in equation (8). In an exact calculation the solvent fow feld follows from solution of Stokes equations with the surfaces of the $N$ colloidal particles in a given conf guration providing the boundary conditions (essentially what is done in Stokesian dynamics simulation $[41,49,50])$. By replacing this solvent velocity feld with the aff ne fow, we neglect the need for the solvent to fow around the particles and are thus able to fully specify the solvent fow prof le from the outset, without requiring that this be determined as part of a self-consistent calculation ${ }^{4}$. If necessary, the assumption

4 Interestingly, the force acting on an isolated hard-sphere of radius $R$ in a Newtonian solvent under (Stokesian) shear fow is identical to that acting on a point particle $(R \rightarrow 0)$ moving with the aff ne fl $\mathrm{w}$; i.e. the fact that the solvent $f$ ows around the particle does not inf uence the resulting force. However, setting $\mathbf{v}(\mathbf{r}, t)=\kappa(t) \cdot \mathbf{r}$ is an approximation for fi ite colloidal volume fraction. 
of purely aff ne fow could be corrected to frst order. For example, under simple shear fow the solvent fow prof le around a single spherical particle is well known [30] and could form the basis of a superposition-type approximation to the full fuctuating velocity f eld.

It is important to note that the assumption of a translationally invariant velocity gradient $\kappa(t)$ is potentially rather severe as it excludes from the outset the possibility of inhomogeneous fow, as observed in shear banded and shear localized states. While physically reasonable for low and intermediate density colloidal fuids, the assumption of homogeneity could become questionable when considering the fow response of dynamically arrested states, for which brittle fracture may preclude plastic $\mathrm{fl} \mathrm{w}^{5}$. Moreover, it is implicit in the approximation $\mathbf{v}(\mathbf{r}, t)=\kappa(t) \cdot \mathbf{r}$ that the imposed fow profle acts instantaneously throughout the system. In experiments where strain or stress are applied at the sample boundaries a f nite time is required for transverse momentum diffusion to establish the velocity feld. Nevertheless, experiments and simulations of the transition from equilibrium to homogeneous steady state fow have shown that a linear velocity profle is established long before the steady state regime is approached, thus suggesting that the assumption of an instantaneous translationally invariant $f$ ow is acceptable for certain colloidal systems [62].

\subsection{Nonequilibrium states}

In equilibrium, the principle of detailed balance asserts that the microscopic probability fux vanishes, $\mathbf{j}_{i}=\Psi \mathbf{d}_{i}(\ln \Psi+$ $\left.\beta U_{\mathrm{N}}\right)=0$, where $U_{\mathrm{N}}$ is the total interparticle potential energy. This balance between conservative and Brownian forces thus yields the familiar Boltzmann-Gibbs distribution $\Psi_{e}=$ $\exp \left(-\beta U_{\mathrm{N}}\right) / Z_{\mathrm{N}}$, where $Z_{\mathrm{N}}$ is the conf gurational part of the canonical partition function. In the presence of fow $(\kappa(t) \neq 0)$ there exists a f nite probability current which breaks the time reversal symmetry of the equilibrium state and detailed balance no longer applies. A nonvanishing probability current thus serves to distinguish between equilibrium and nonequilibrium solutions of $(14)$ and rules out the possibility of a BoltzmannGibbs form for the nonequilibrium distribution. While such a Boltzmann-Gibbs distribution is clearly inadequate for nonpotential fows (e.g. simple shear), for potential fows (e.g. planar elongation) it is perhaps tempting to assume such a distribution by employing an effective 'fow potential' $U_{\mathrm{f}}$ (see e.g. [63]). The fundamental error of assuming an "effective equilibrium' description of nonequilibrium states is made very clear by the non-normalizability of the assumed distribution $\Psi \sim \exp \left(-\beta\left(U_{\mathrm{N}}+U_{\mathrm{f}}\right)\right)$. These considerations serve to emphasize the fact that the only true way to determine the distribution function for systems under fow is to solve the Smoluchowski equation (14).

5 In [59] a confocal microscopy study of polymethyl methacrylate (PMMA) colloids suspended in a mixture of decalin and tetralin revealed inhomogeneous plug fow for glassy states. In contrast, the rheological experiments performed in [60] and [6]] using thermosensitive PNIPAM coreshell particles are fully consistent with homogeneous $f$ ow, both above and below the glass transition.
For much of the present work we will focus on the response of colloidal dispersions to steady fows. While experiment and simulation clearly demonstrate that well defi ed steady states may be achieved following a period of transient relaxation, it is interesting to note that there exists no mathematical proof of a Boltzmann $\mathrm{H}$-theorem for equation (14) which would guarantee a unique long-time solution for the distribution function. The absence of an H-theorem for colloidal dispersions under steady fow is a consequence of the hard repulsive core of the particles which invalidates the standard methods of proof generally applied to Fokker-Planck-type equations [19, 46].

A further nontrivial aspect of equation (14) emerges when considering the translational invariance properties of the timedependent distribution function $\Psi(t) \equiv \Psi\left(t,\left\{\mathbf{r}_{i}\right\}\right)$, achieved by shifting all particle coordinates by a constant vector $\mathbf{r}_{i}^{\prime}=$ $\mathbf{r}_{i}+\mathbf{a}$ (see section 7.3 for more details). For an arbitrary incompressible fow it has been proven that a translationally invariant initial distribution function leads to a translationally invariant, but anisotropic distribution function $\Psi(t)$, despite the fact that the Smoluchowski operator [14] generating the dynamics is itself not translationally invariant [17]. Although the proof outlined in [17] omitted hydrodynamic interactions, it may be expected that the same result holds in the presence of hydrodynamics due to the dependence of the diffusion tensors on relative particle coordinates.

\section{Quiescent states}

\subsection{Hard-spheres}

Theoretical and simulation studies based on equation (14) have focused largely on the hard-sphere model. In addition to being mathematically convenient, the focus on this simple model is motivated largely by the availability of well characterized hard-sphere-like experimental colloidal systems [7]. In the absence of $f$ ow, a system of monodisperse hard-sphere colloids remain in a disordered $f$ uid phase up to a volume fraction of $\phi=0.494$, beyond which they undergo a frst order phase transition to a solid phase of $\phi=0.545$ with face-centredcubic order (see fgure 3). This unexpected, entropically driven, ordering transition was f rst observed using molecular dynamics computer simulation in the late 1950s [64] and remains a current topic of both experimental and theoretical research (for a recent review see [65]).

Making the system slightly polydisperse frustrates crystalline ordering and suppresses the freezing transition. In suff ciently polydisperse systems $s^{6}$ a disordered fuid remains in the equilibrium state up to a volume fraction $\phi \approx$ 0.58 , at which point the dynamics becomes arrested and a colloidal glass state is formed. This dynamical transition to a non-ergodic solid is characterized by a non-decaying intermediate scattering function at long times for which dynamic light scattering results $[7,8]$ are well described

6 In order to observe glassy behaviour the timescale of crystal nucleation $\tau_{f f}$ has to be extended beyond the accessible experimental or simulation measurement time. Fortunately, $\tau_{\mathrm{fr}}$ is a sensitive function of the degree of polydispersity and for hard-sphere-like systems a polydispersity $\sim 5 \%$ is usually suff cient to effectively supress crystallization. 


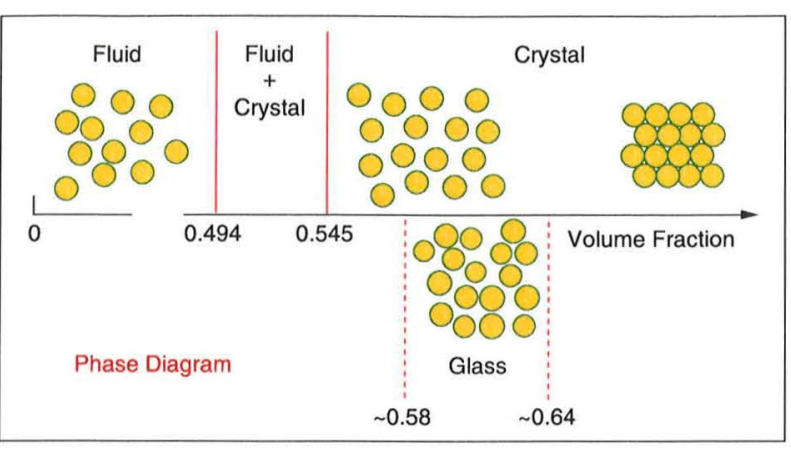

Figure 3. A schematic illustration of the phase diagram of hard-spheres as a function of volume fraction. Monodisperse systems undergo a freezing transition to an FCC crystal with coexisting densities $\phi=0.494$ and 0.545 . Polydispersity suppresses the freezing transition resulting in a glass transition at $\phi \sim 0.58$, which lies below the random-close-packing value of $\phi \sim 0.64$.

by the mode-coupling theory (MCT) [10]. The standard quiescent MCT consists of a nonlinear integro-differential equation for the transient density correlator which exhibits a bifurcation, identif ed as a dynamic glass transition, for certain values of the system parameters [10]. One of the appealing aspects of MCT is the absence of adjustable parameters: all information regarding both the particle interaction potential and thermodynamic state point enter via the static structure factor, which is assumed to be available from either independent measurements or equilibrium statistical mechanical calculations. For monodisperse hard-spheres, MCT predicts a dynamic glass transition at $\phi \approx 0.516$ when the Percus-Yevick [15] approximation is used to generate the structure factor, although other values may be obtained using either alternative theories, simulation or experiment to determine the static equilibrium structure [66]. We note that using MCT together with Percus-Yevick structure factors enables a glass transition to be studied for monodisperse hard-spheres at volume fractions above freezing. Neither MCT nor PY theory is capable of incorporating crystalline ordering effects and both implicitly assume an amorphous microstructure.

A shortcoming of the quiescent MCT is that it predicts an idealized glass transition with a divergent structural relaxation time and does not incorporate the activated processes which in experiment and simulation studies are found to truncate the divergence. While extensions of MCT aiming to incorporate additional relaxation channels have been proposed $[67,68]$, the underlying microscopic mechanisms remain unclear. Despite its mean-fie d character, the MCT does capture some aspects of the heterogeneous dynamics [69-72] which have been observed using confocal microscopy [73].

Finally, we note that a similar scenario of crystallization and dynamical arrest may be observed also in two-dimensional systems $[65,74]$. Despite the reduced dimensionality and new physical mechanisms associated with melting in two dimensions (where the hexatic phase plays an important role) the phase diagram for both monodisperse and polydisperse hard-disc systems is qualitatively identical to the threedimensional case illustrated in f gure 3. The close analogies between two- and three-dimensional systems may be exploited when considering nonequilibrium situations for which numerical calculations in 3D may prove prohibitively time consuming [75]. Viewing a binary mixture as the simplest form of polydispersity, MCT has been employed to study the inf uence of 'mixing' (variations in composition and size ratio) on the glass transition of three-dimensional hard-sphere [76] and two-dimensional hard-disc [77] systems. These studies have revealed intriguing connections between glassy arrest and random-close-packing.

\subsection{Attractive spheres}

The addition of an attractive component to the hard-sphere potential can lead to an alternative form of dynamical arrest to either a gel at low volume fraction $[78,79]$ or an attractive glass state at higher volume fractions [80, 81] when the interparticle attraction becomes suff ciently strong. The origins of the attractive interaction are various, e.g. van der Waals forces [6] or the depletion effect when non-adsorbing polymer is added to a dispersion [82-84]. This form of dynamical arrest has been investigated experimentally using both dynamic light scattering (see e.g. [79-81]) and confocal microscopy (see e.g. [85]). There is now compelling evidence both from experiment [86] and simulation [87] that for f nite densities gelation occurs via a process of arrested phase separation and that only for very dilute, strongly attractive, suspensions does this mechanism cross over to one of diffusion limited aggregation.

When applied to attractive colloidal systems the MCT predicts a nonequilibrium 'phase diagram' which is in good agreement with the results of experiment and qualitatively describes the phase boundary separating fuid from arrested states as a function of volume fraction and attraction strength [80, 81]. Recent studies of systems in which the depletion attraction between particles is complemented by the addition of a competing long range electrostatic repulsion [88] have revealed a rich and unexpected phase behaviour, including stable inhomogeneous phases [89] and metastable arrested states [90]. In addition, impressive new developments in colloid chemistry have enabled the construction of "colloidal molecules' in which the particle surface is decorated with a prescribed number of attractive sites, thus rendering the total interaction potential anisotropic [91]. For a review of these more recent developments we refer the reader to [92].

\section{Rheological phenomenology}

As noted in the introduction, dispersions of spherically symmetric colloidal particles exhibit a diverse range of response to externally applied fow. Much, although not all $^{7}$, of the generic rheological behaviour of colloidal dispersions is captured by the hard-sphere model introduced in section 4.1. In order to focus the discussion we will consider the special case of hard-spheres subject to a steady shear $\mathrm{fl} \mathrm{w}$. In fi ure 4 we show the results of stress controlled experiments performed

${ }^{7}$ For example, the yielding behaviour of attractive colloids is considered in [93]. 


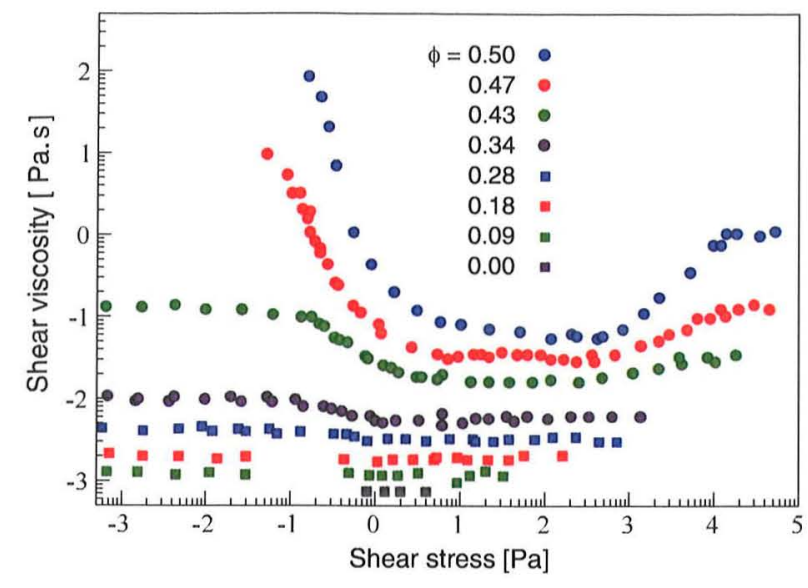

Figure 4. The shear viscosity of an aqueous dispersion of colloidal latex as a function of the externally applied shear stress. Data for a range of volume fractions are shown, from dilute up to a dense colloidal liquid at $\phi=0.50$. Shear thinning is evident at intermediate stress values as the viscosity of the dispersion decreases due to ordering of the particles by the fow. At larger applied stresses, for suff ciently high volume fraction, the dispersion shear thickens as hydrodynamic lubrication forces lead to cluster formation and increased disorder. (Figure adapted from [94].)

on a dispersion of spherical latex particles dispersed in water at various volume fractions, ranging from a dilute 'colloidal gas' up to $\phi=0.5$, corresponding to a dense colloidal liquid state close to the freezing transition [94]. We note that for the experimental steady shear fow data shown in fgure 4 it is not signif cant that the shear stress is employed as the control parameter dictating the $\mathrm{fl} \mathrm{w}$. The quiescent system is ergodic at all considered state points and qualitatively identical results may thus be expected in an analogous strain controlled experiment, provided that the fow remains homogeneous.

\subsection{Zero-shear viscosity}

For each of the volume fractions shown in fgure 4 the shear viscosity $\eta \equiv \sigma_{x y} / \dot{\gamma}$ is constant for small applied stresses (corresponding to small shear rates) and def nes the zero-shear viscosity $\eta_{0}$. The data shown in fgure 4 clearly demonstrate that the addition of colloidal particles leads to a dramatic increase of $\eta_{0}$ above that of the pure solvent (note the logarithmic scale in f gure 4).

From a theoretical perspective, there are two alternative ways to understand the increase of $\eta_{0}$ as a function of $\phi$. The frst is to relate the viscosity to the fow distorted pair correlation functions in the limit of vanishing fow rate (see section 6.1). The leading order anisotropy of $g(\mathbf{r}, P e \rightarrow 0)$ captures the perturbing effect of weak fow on the microstructure and thus describes the increase of $\eta$ in terms of temporally local and physically intuitive correlation functions. The second method, referred to as either the 'time correlation' or 'Green-Kubo' approach, provides an equally rigorous method in which the viscosity is expressed as a time integral over a transverse stress autocorrelation function (see section 7). Although the two approaches are formally equivalent, it is the latter which enables a direct connection to be made between $\eta_{0}$ and the timescale describing the collective relaxation of the microstructure.

Within the Green-Kubo formalism the thermodynamic colloidal contribution to the zero-shear viscosity is given by [191]

$$
\eta_{0} \equiv \begin{gathered}
\sigma_{x y} \\
\dot{\gamma}
\end{gathered}=\int_{0}^{\infty} \mathrm{d} t G_{\mathrm{eq}}(t)
$$

where the equilibrium shear modulus is formally def ned as a stress autocorrelation function

$$
G_{\mathrm{eq}}(t)={ }_{k_{\mathrm{B}} T V}^{1}\left\langle\hat{\sigma}_{x y} \mathrm{e}^{\Omega_{\mathrm{eq}}^{\dagger} t} \hat{\sigma}_{x y}\right\rangle,
$$

where $V$ is the system volume and $\Omega_{\mathrm{eq}}^{\dagger}$ is the equilibrium adjoint Smoluchowski operator [14]. The fuctuating stress tensor element is given by $\hat{\sigma}_{x y} \equiv-\sum_{i} F_{i}^{x} r_{i}^{y}$, and the average is taken using the equilibrium Boltzmann-Gibbs distribution.

Equation (17) is an exact Green-Kubo relation which expresses a linear transport coeff cient, in this case the shear viscosity, as an integral over a microscopic autocorrelation function. For dense colloidal dispersions the shear modulus starts from a well def ned initial value ${ }^{8}$ from which it rapidly decays on a timescale set by $d^{2} / D_{0}$ to a plateau. For much later times the modulus decays further from the plateau to zero, thus identifying the timescale of structural relaxation $\tau_{\alpha}$ (see f gure 5). The 'two step' decay of the time-dependent shear modulus is a generic feature of interacting systems exhibiting both a rapid microscopic dynamics and a slower, interaction induced, structural relaxation and is familiar from experiments and simulations of both colloidal and polymeric systems (where the Fourier transform $G^{*}(\omega)$ is typically considered, rather than $G(t)$ directly).

Within the idealized mode-coupling theory (MCT) the equilibrium shear modulus (18) is approximated by [96]

$$
G_{\text {eq }}(t)=\begin{gathered}
k_{\mathrm{B}} T \\
60 \pi^{2} d^{3}
\end{gathered} \int_{0}^{\infty} \mathrm{d} k k^{4}\left(\begin{array}{c}
S_{k}^{\prime} \\
S_{k}
\end{array}\right)^{2} \Phi_{k}^{2}(t),
$$

where $T$ is the temperature, $S_{k}$ and $S_{k}^{\prime}$ are the static structure factor and its derivative, respectively, and $\Phi_{k}(t)$ is the transient density correlator def ned by

$$
\Phi_{k}(t)=\frac{1}{S_{k}}\left\langle\rho_{k}^{*}(t) \rho_{k}(0)\right\rangle,
$$

where $\rho_{k}=\sum_{j} \exp \left(\mathrm{ik} \cdot \mathbf{r}_{j}\right)$. The collective coordinates $\rho_{k}$ are the central quantity within mode-coupling approaches and their autocorrelation (20) describes the temporal decay of density fuctuations which slow and ultimately arrest as the glass transition is approached. The mode-coupling approximation (19) arises from projection of the dynamics onto density-pair modes and thus expresses the relaxation of stress fuctuations in terms of density fuctuations. Within MCT

8 In general, the initial value of the shear modulus is determined by near-f eld hydrodynamic lubrication forces. The commonly studied case of Brownian hard-spheres in the absence of hydrodynamic interactions is a pathological special case for which $G(t \rightarrow 0) \sim t^{-\frac{1}{2}}$ as a consequence of the discontinuous potential interaction. Nevertheless, the Brownian hard-sphere $G(t)$ rapidly becomes physical for later times. We refer the reader to [95] for more details on this issue. 


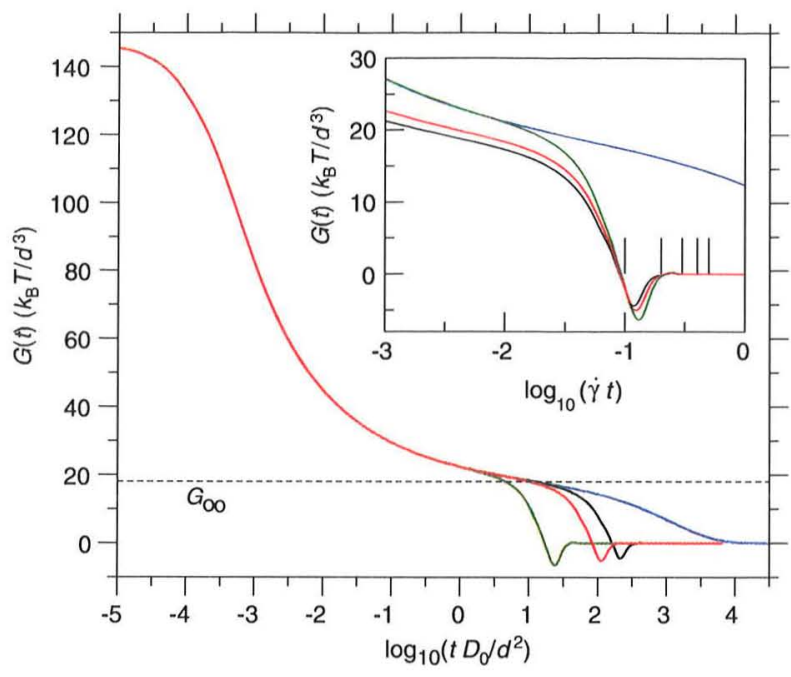

Figure 5. The generalized shear modulus $G(t) \equiv G(t, P e)$ at a volume fraction relative to the glass transition $\phi-\phi_{\mathrm{g}}=-1.16 \times 10^{-3}$ calculated using the extended mode-coupling approach [62]. The f nal relaxation of the equilibrium modulus (blue curve) serves to defin the alpha relaxation timescale. As the applied shear rate is increased the relaxation timescale is reduced. Curves are shown for $P e=5.5 \times 10^{-3}$ (green), $1.1 \times 10^{-3}$ (red) and $5.5 \times 10^{-4}$ (black). The plateau value which develops for volume fractions approaching the glass transition is indicated by the broken line. The inset shows the same data as a function of strain $\dot{\gamma} t$ (Reproduced with permission from [62]. Copyright 2008, IOP Publishing.)

the correlator is approximated by the solution of a nonlinear integro-differential equation

$$
\dot{\Phi}_{\mathrm{q}}(t)+\Gamma_{\mathrm{q}}\left[\Phi_{\mathrm{q}}(t)+\int_{0}^{\infty} \mathrm{d} t^{\prime} m_{\mathrm{q}}\left(t-t^{\prime}\right) \dot{\Phi}_{\mathrm{q}}\left(t^{\prime}\right)\right]=0,
$$

where $\Gamma_{\mathrm{q}}=q^{2} / S_{\mathrm{q}}$ and the memory function is a quadratic functional of $\Phi_{k}(t)$ which depends upon both volume fraction and the static structure factor (which serves as proxy for the pair interactions). Explicit expressions for the quiescent memory function may be found in [98]. Equation (21) predicts that $\tau_{\alpha}$ diverges at the glass transition volume fraction which then leads, via equations (17) and (19), to a corresponding divergence of $\eta_{0}$. While in many cases the quiescent MCT gives a good account of experimental data [11] the precise nature and location of this apparent divergence remains a matter of debate (see e.g. [97]).

\subsection{Shear thinning}

Turning again to f gure 4 it is evident that for a given volume fraction the viscosity decreases as a function of shear rate. This shear thinning behaviour typically sets in when the shear rate begins to exceed the inverse of the timescale governing structural relaxation, that is for values of the Weissenberg number $W i \equiv \dot{\gamma} \tau_{\alpha}>1$, where $\dot{\gamma}$ is the characteristic rateof-strain and $\tau_{\alpha}$ is the structural relaxation time. Within the range $0<W i<1$ the system is within the linear response regime and the fow rate is suff ciently slow that the collective relaxation of the microstructure, characterized by, e.g., the decay of the transient density correlator (20), is not infl enced.

For $W i>1$ the rate of structural relaxation is enhanced by the fow feld. The modulus thus becomes a function of the shear rate and the viscosity shear thins. To incorporate this nonlinear response equation (17) may be generalized to

$$
\eta(P e) \equiv \begin{gathered}
\sigma_{x y}(P e) \\
\dot{\gamma}
\end{gathered}=\int_{0}^{\infty} \mathrm{d} t G(t, P e),
$$

where the functional dependence on $P e$ has been made explicit. The nonlinear modulus is thus def ned as

$$
G(t, P e)={ }_{k_{\mathrm{B}} T V}^{1}\left\langle\hat{\sigma}_{x y} \mathrm{e}^{\Omega^{\dagger} t} \hat{\sigma}_{x y}\right\rangle
$$

where $\Omega^{\dagger}$ is the adjoint Smoluchowski operator generating the particle dynamics [14]. Despite the equilibrium averaging employed in (18), it is important to note that an initial stress fuctuation $\sigma_{x y}$ evolves to a $\mathrm{fl}$ ctuation at later time $t$ under the full dynamics, including the effects of fow. This serves to distinguish the transient stress correlator (18) from that which would naturally be measured in a computer simulation, where all averaging is performed with respect to the full nonequilibrium distribution function. In the absence of fow $\Omega^{\dagger}=\Omega_{\mathrm{eq}}^{\dagger}$ and the equilibrium result (18) is recovered.

Recent generalizations of the mode-coupling theory [20] provide approximate expressions for the nonlinear modulus $G(t, P e)$, see section 7 . These approaches incorporate the effects of shear fow into the memory kernel responsible for slow structural relaxation and describe the speeding up of the relaxational dynamics. In fgure 5 we show $G(t, P e)$ calculated using the generalized MCT [62] for volume fractions close to (but below) the glass transition at various values of the shear rate. For $W i<1$ the equilibrium result (blue curve) is not inf uenced by the fow. However, for $W i>1$ the longest relaxation time becomes dictated by the fl $\mathrm{w}$ and $\tau_{\alpha} \sim \dot{\gamma}^{-1}$, as is demonstrated by the inset to f gure 5 which shows the same data as a function of strain. From equation (22) it is clear that within this generalized GreenKubo approach the decrease of $\tau_{\alpha}$ with increasing $\dot{\gamma}$ results in shear thinning of the viscosity.

An alternative, although equally valid, viewpoint is provided by approaches focusing on the fow distorted pair correlations. Exact results for low volume fraction dispersions based on the pair Smoluchowski equation (see section 6.1) have shown that shear thinning results from a decrease in the Brownian contribution to the shear stress [56]. In dispersions at higher volume fraction the reduction in the Brownian stress is manifest in an ordering of the particles in the direction of fow which serves to reduce the frequency of particle collisions. Within the shear thinning regime evidence for layered or string-like ordering in intermediate volume fraction systems has been provided by Brownian dynamics simulations [99, 100] and, albeit with different characteristics, by Stokesian dynamics simulations which include hydrodynamic interactions [49, 101-103]. Interestingly, simulations have also shown that the fow induced order continues to develop following its initial 
onset. This 'ripening' of the ordered phase leads to a timedependence of the viscosity known as thixotropy [102-105] and complicates the determination of fow curves in both simulation and experiment. For each shear rate the measurement time must be suff ciently long that the viscosity saturates to a plateau value before the shear rate is updated.

We note that the same ordering mechanism discussed here for colloidal dispersions would also lead to an analogous shear thinning scenario for atomic liquids (e.g. liquid argon). In this case, however, the shear rates required to observe such nonNewtonian rheology are several orders of magnitude larger than those readily accessible in experiment. For this reason, non-Newtonian effects in atomic systems remain largely a matter of academic interest.

\subsection{Shear thickening}

Following the regime of shear thinning, a second Newtonian plateau develops for which the viscosity attains an approximately constant value as a function of shear rate and the fow induced ordering of the system continues to develop. At higher shear rates the viscosity undergoes a rapid increase once a critical value of the shear stress is exceeded. Such shear thickening behaviour can be either continuous [58] or discontinuous [106] in character and is somewhat counterintuitive in light of the discussion presented in section 5.2 regarding fow induced microstructural ordering and its connection to shear thinning. Suspensions of nonaggregating particles at intermediate volume fractions generally show reversible shear thickening, however the details of the increase in viscosity depend upon the details of the system (particle-type, solvent, etc) as well as the thermodynamic control parameters [107].

In section 5.2 we noted that the onset of shear thinning occurs for values of the Weissenberg number $W i>1$, ref ecting the essential competition between fow and structural relaxation. In contrast, the onset of shear thickening behaviour is determined by the value of the bare Peclet number $P e$, thus serving to highlight the different mechanisms dominating the physics of thinning and thickening states. In experiment, the difference in scaling of $W i$ and $P e$ with particle diameter $d$ enables the extent of the second Newtonian plateau separating shear thinning and shear thickening regimes to be controlled as a function of particle size. When the microstructure under fow is also of interest, the constraints of instrumental resolution (in e.g. confocal microscopy) place additional limits on the particle size which have also to be taken into consideration.

The earliest theoretical explanations of shear thickening in colloidal dispersions proposed that the observed viscosity increase is the consequence of an order-to-disorder transition [108, 109]. Within this picture, the ordered planes of particles which form within the shear thinning regime, and which persist throughout the second Newtonian viscosity plateau, begin to interact via hydrodynamic coupling at suff ciently high shear rates. This interaction pulls particles out of the layers, leading to increased particle collisions, disorder, and a consequent increase in viscosity. The onset of shear thickening is thus identif ed with the hydrodynamic instability of a layered microstructure (see [110] for a discussion of this issue).

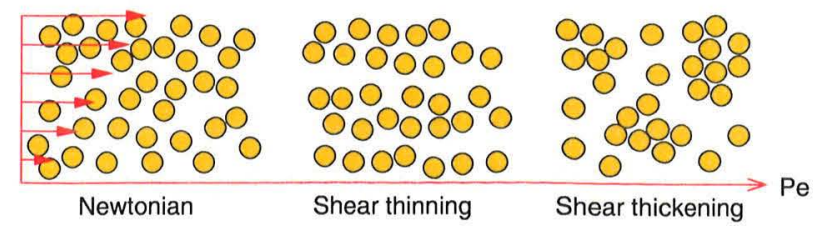

Figure 6. Schematic illustration of the microstructural order and disorder induced in a dense colloidal dispersion by shear $\mathrm{fl} \mathrm{w}$. At low values of $P e$ (leftmost conf guration) the viscosity remains constant as diffusion is able to restore the equilibrium microstructure more rapidly than the shear $\mathrm{fl} \mathrm{w}$ can disrupt it. At intermediate shear rates (central configura ion) the rate of shear exceeds the rate of structural relaxation, $W i>1$ leading to microstructural ordering and shear thinning. At high shear rates (right configura ion) hydrodynamic lubrication forces lead to particle clustering which strongly enhances the hydrodynamic contribution to the viscosity and result in shear thickening.

Despite the intuitive appeal of interpreting shear thickening as an order-to-disorder transition, questions were raised by the experiments of $[110,111]$ in which a specifi system (electrostatically stabilized Latex particles in glycols) was found to display shear thickening in the absence of an ordered phase. These results suggested that while an ordered phase may well precede the shear thickening regime as the shear rate is increased, it is not a necessary prerequisite. According to these f ndings, shear thickening occurs via an independent physical mechanism and is not simply related to a loss of microstructural order. Further insight into the microscopic mechanism underlying shear thickening was provided by Stokesian dynamics simulations [99, 112] which identifed the formation of hydrodynamically bound particle clusters at high shear rates. Such 'hydroclusters' form when the shear fow is suff ciently strong that the particle surfaces are driven close together. At such small separations the hydrodynamic lubrication forces dramatically reduce the relative mobility of the particles such that they remain trapped together in a bound orbit (a point which we will later revisit in section 6.2). Transient shear-driven hydroclusters would appear to be the def ning feature of shear thickened states and experimental evidence for their importance is accumulating [113]. Nevertheless, opinion remains divided regarding the fundamental mechanisms at work [114].

The hydrodynamic mechanisms described above give rise to a continuous, albeit rapid, rise in the viscosity as a function of shear rate. An alternative scenario may arise when, at some critical value of the shear rate, the viscosity exhibits a discontinuous jump as the system becomes jammed [58, 108, 115-117]. While it is anticipated that hydrodynamics will be relevant for the description of shear thickening at intermediate volume fractions (e.g. $0<\phi<$ 0.5 , as considered in fgure 4) alternative mechanisms may become important upon approaching the glass transition. In $[118-120]$ a 'schematic' mode-coupling theory similar to those to be discussed in section 7 was developed, in which a coupling to stress was introduced into the nonlinear equations determining the decay of the transient density correlator. Upon varying the model parameters a range of 
rheological behaviour was revealed, including both continuous and discontinuous shear thickening, as well as a jamming transition to a non-ergodic solid state. Within this picture, shear thickening and jamming are viewed as a type of stress induced glass transition, for which the applied stress inhibits particle motion, even in the absence of hydrodynamics. A number of works have suggested a relationship between shear thickening and jamming $[3,4,103,121]$ although details of the connection between hydrodynamic cluster formation and jamming transitions of the kind more familiar from studies of granular media [122] remain unclear.

The addition of an attractive component to the strongly repulsive colloidal core can lead to gel formation and irreversible focculation (see section 4.2). For such systems shear thickening is generally not observed, as the increase in the hydrodynamic contribution to the viscosity with increasing shear rate is more than compensated for by the decrease in the thermodynamic contribution arising from the attraction (see e.g. [123]). The generic behaviour of gel and foc states is thus monotonic shear thinning as a function of shear rate [107]. It is therefore surprising that recent experiments using attractive carbon black particles [124] have identif ed a rich shear thickening behaviour for which the viscosity increases beyond a critical value of the applied shear stress. In this case an additional physical mechanism has been proposed by which the forces exerted by shear $\mathrm{fl} \mathrm{w}$ cause $\mathrm{fl}$ cs to break apart, leading to an increased surface area and thus greater hydrodynamic dissipation [124].

As pointed out in section 5.2, shear thinning is not unique to colloidal systems and can also be observed, albeit at high shear rates, in simple atomic liquids. In contrast, shear thickening of the type discussed above is not found in atomic systems (for which the 'solvent' is a vacuum) and demonstrates clearly the breakdown of the correspondence between colloidal and simple liquids for strongly nonequilibrium states. Indeed it is quite clear that the view of colloids as 'big atoms' [125] will only hold in situations for which the inf uence of the solvent is negligible and that new physics may emerge when the role of hydrodynamic interactions becomes signif cant. We note that an alternative type of shear thickening has been observed at high shear rates in molecular dynamics simulations of simple liquids [126-129]. In these simulations a prof le unbiased thermostat was employed to remove artefacts which may arise when a linear $\mathrm{fl} \mathrm{w}$ profi e is assumed. Shear thickening was observed in simulations performed at constant volume, but not in those performed at constant pressure.

Finally, we would like to note that the onset of shear thickening at high $\mathrm{fl} \mathrm{w}$ rates has been associated with unexpected behaviour of the frst normal stress difference $N_{1}=\sigma_{x x}-\sigma_{y y}$. Typically, dispersions at low or moderate shear rate exhibit a positive value of $N_{1}$, indicating that a Weissenberg (or 'rod climbing') effect would be observed in shear experiments performed in a Couette geometry [2]. Experiments on dense colloidal dispersions with repulsive interactions $[116,130,131]$ have revealed that $N_{1}$ can change sign from positive to negative upon increasing the fow rate into the regime where the viscosity shear thickens. Similar behaviour has been observed in Stokesian
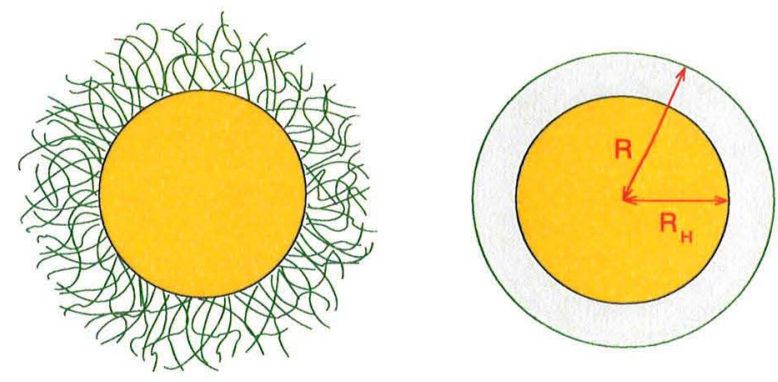

Figure 7. Many commonly studied colloidal particles (e.g. PNIPAM) consist of a polymeric core grafted with a layer of polymer (schematically represented on the left) which serves to stabilize against focculation. Given a suff ciently dense and crosslinked polymer brush the particles exhibit a strongly repulsive effective potential interaction approximating that of hard-spheres with radius $R$. The ability of the solvent to penetrate into the brush results in a hydrodynamic radius $R_{\mathrm{H}}<R$. These effects may be mimicked by a simple hard-sphere model (sketch on the right) in which $R / R_{\mathrm{H}}$ can be used to control the influenc of hydrodynamic interactions.

dynamics simulations [132] and in numerical solutions of the Smoluchowski equation for dilute systems [133]. In contrast to these results for purely repulsive interactions, recent experiments on attractive focculated colloidal dispersions display a monotonically increasing $N_{1}$ throughout the shear thickening regime [124].

\subsection{Yield stress}

For colloidal f uid states $(0<\phi<0.494)$ the data presented in $\mathrm{f}$ gure 4 represent the generic phenomenology of dispersions of strongly repulsive colloids under shear fow. In fact, this behaviour is not limited to simple shear. Qualitatively identical behaviour is found in Stokesian dynamics simulations for the extensional viscosity [22] of dispersions of hard-sphere colloids under steady extensional fow, with the shear rate replaced by the rate of Hencky strain [52].

As already noted in section 5.1 , increasing the volume fraction of a colloidal liquid leads to a strong increase in the zero-shear viscosity. Assuming that crystallization has been suppressed by polydispersity, the volume fraction can then be further increased, eventually resulting in an apparent divergence of the zero-shear viscosity, either at the glass transition volume fraction (according to mode-coupling theory $[10,96])$, or some higher volume fraction approaching random-close-packing [97]. The variation of the viscosity as a function of shear rate for volume fractions ranging from 0.45 to 0.57 is demonstrated in more detail by the data shown in f gure 8 . These experiments were performed on a system of poly(ethylene glycol)-grafted polystyrene colloidal particles dispersed in water [134]. For the two lowest volume fractions considered $(\phi=0.45$ and 0.48$)$ a clear zero-shear viscosity may be identif ed from the low shear rate plateau, with shear thinning evident at higher shear rates for $\phi=0.48$. As the volume fraction is increased above 0.48 the low shear rate plateau moves to smaller rates, out of the experimental window of resolution, and the dispersion shows shear thinning over the entire range. Analysis of the intensity correlation function 


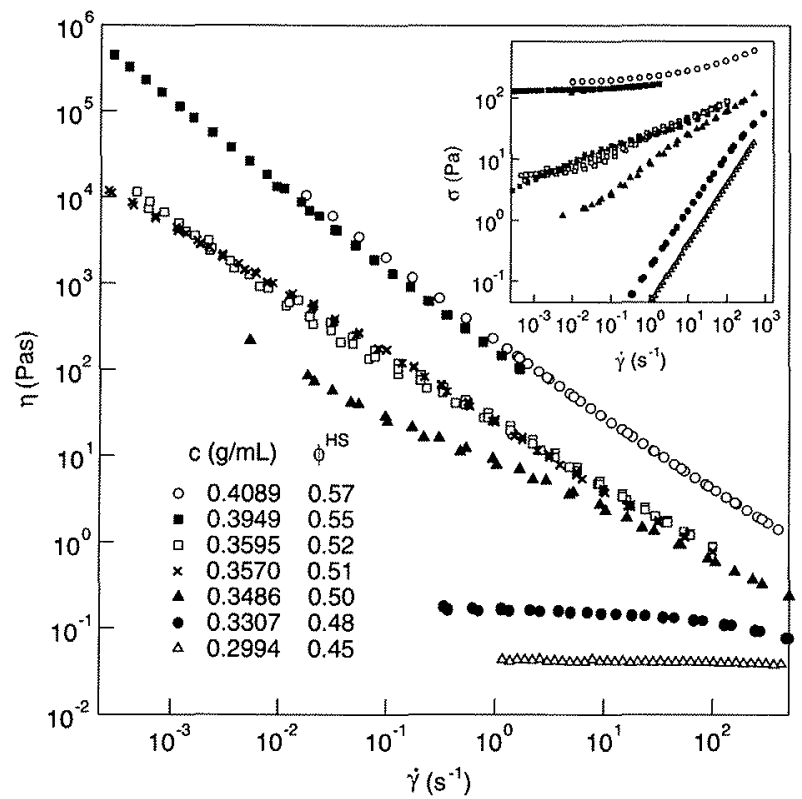

Figure 8. Shear thinning and the dynamic yield stress of a concentrated aqueous dispersion of poly(ethylene glycol)-grafted polystyrene colloidal particles which, to a good approximation, behave as hard-spheres. The main $f$ gure and inset show the viscosity and shear stress, respectively, as a function of shear rate. (Reprinted with permission from [134]. Copyright 2005 by the American Physical Society.)

(related to the transient density correlator (20)) measured using dynamic light scattering leads to an estimate of the glass transition for this system of $0.53<\phi_{\mathrm{g}}<0.55$, somewhat lower than the typical value $\phi_{\mathrm{g}} \sim 0.58$ obtained for PMMA hard-sphere-like colloids. The viscosity data for the two highest volume fractions ( $\phi=0.55$ and 0.57 ) are consistent with a divergence in the zero-shear viscosity at the glass transition, as predicted by the MCT (see section 5.1).

According to the extended MCT $[20,57,135]$, for glassy states the slowest relaxation time is $\tau_{\alpha} \sim \dot{\gamma}^{-1}$, which leads, via equation (22), to $\eta \sim G(t \rightarrow \infty) \dot{\gamma}^{-1}$, where $G(t \rightarrow \infty)$ is the plateau modulus (see f gure 5 ), thus reproducing the power law decay of the viscosity demonstrated by the data in $f$ gure 8 . For the idealized glassy states considered by MCT, where $\tau_{\alpha}$ is inf nite in the absence of fow, this power law dependence extends to the limit $\dot{\gamma} \rightarrow 0$, resulting in a true divergence. In real colloidal experiments, higher order relaxation processes will always endow the quiescent system with a f nite value of $\tau_{\alpha}$ and the viscosity divergence will be truncated. The low shear divergence of the viscosity and power law shear thinning $\eta \sim \dot{\gamma}^{-1}$ suggested by f gure 8 are supported by independent experiments performed on thermosensitive coreshell particles $[60,61]$. However, some recent experiments on sterically stabilized PMMA particles provide contradictory evidence and have suggested a nontrivial dependence of the relaxation time on shear rate, namely $\tau_{\alpha} \sim \dot{\gamma}^{-0.8}$, which remains to be understood [136].

The inset to fgure 8 shows the shear stress as a function of shear rate and provides an alternative representation of the viscosity data shown in the main panel. For the two highest volume fraction samples ( $\phi=0.55$ and 0.57$)$ the shear stress becomes constant for the lowest shear rates considered, thus identifying a dynamic yield stress for glassy states. Both the shear thinning as a function of $P e$ and appearance of a dynamic yield stress as a function of $\phi$ evidenced by fi ure 8 are well described by the extended MCT $[60,61]$.

The relationship between the dynamic yield stress and the more familiar static yield stress mirrors that between stick and slip friction in engineering applications $\left(\sigma_{y}^{\text {stat }}>\sigma_{y}^{\text {dyn }}\right.$ is thus to be expected). Indeed, it may be argued that the dynamic yield stress is, in fact, a more well def ned quantity than the static yield stress. The latter is typically def ned as the step stress amplitude which must be exceeded such that the system will fow at long times? ${ }^{9}$. The point of static yield may therefore be dependent upon details of the system preparation, with the consequence that nonstationary properties, such as sample age in colloidal glasses, could inf uence the outcome of a given experiment ${ }^{10}$. Moreover, the existence of creep motion, for which the strain increases sublinearly with time, makes diff cult an unambiguous identif cation of the static yield stress. In contrast, the dynamic yield stress is def ned as the limiting stress within a sequence of ergodic, fuidized steady states and is thus independent of prior sample history.

It is apparent from equation (22) that a dynamic yield stress can only exist in the event that $\tau_{\alpha} \sim \dot{\gamma}^{-v}$, with $\nu=1$. Values of $v$ less than unity result in a shear stress $\sigma_{x y}(\dot{\gamma} \rightarrow 0)=0$, despite the fact that the viscosity diverges. We thus note that a low shear rate divergence of the viscosity is a necessary but not suff cient condition for the existence of a yield stress. While the results presented in [136] apparently cast doubts on the existence of a dynamic yield stress for certain colloidal glasses, complications due to inhomogeneous, shear localized fow make this a subject of ongoing debate [137].

The interplay between static and dynamic yield has been investigated in simulation studies of a glass forming binary Lennard-Jones mixture (the Kob-Anderson model) using molecular dynamics simulations $[138,139]$. In these simulations the mixture was conf ned between two atomistic walls, one of which was then subjected to either a constant stress or constant strain in order to induce shear fow. It is important to note that due to the application of shear through the boundaries, the fow prof le within the conf ned f uid/glass is an output of the numerical calculation and is not constrained to be linear.

In fgure 9 we show the simulated fow curve for this system (analogous to that shown in the inset to f gure 8) for a glassy statepoint, calculated by applying a fxed rate-ofstrain to one of the bounding walls $[138,139]$. When the

9 We note that there exist numerous alternative def nitions of the "yield stress' in the literature. Two common choices are: (i) the maximum value of the stress overshoot which occurs in response to the onset of steady shear fow, (ii) the point for which $G^{\prime}=G^{\prime \prime}$ as a function of amplitude for $f$ xed frequency in oscillatory experiments (strain sweeps).

${ }^{10}$ This expectation is supported by the simulations performed in [138] which found that the maximum of the stress-strain curve during start-up shear fow, often identifie as the static yield stress, exhibits a logarithmic dependence on the age of the system 


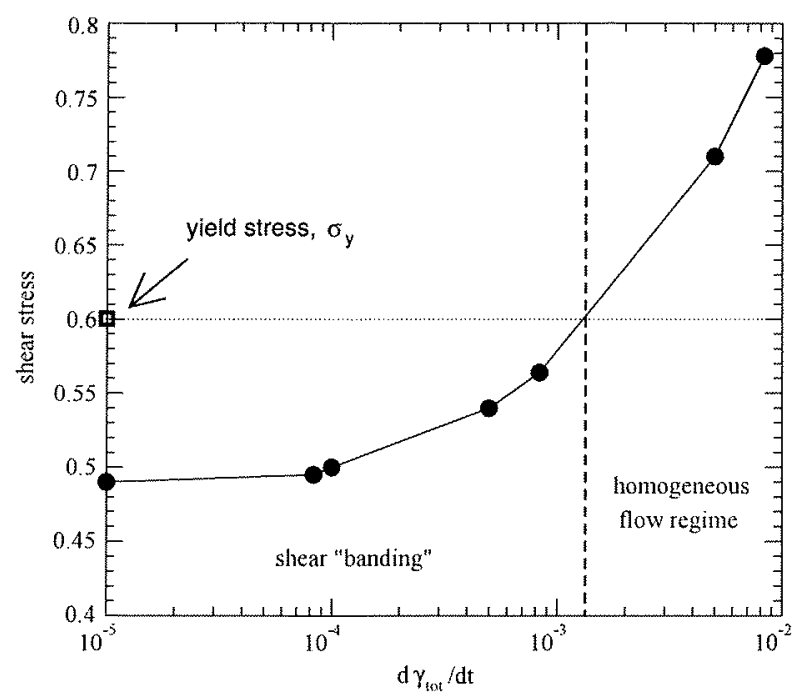

Figure 9. Molecular dynamics simulation results for a glass forming binary Lennard-Jones mixture. The full line shows the shear stress as a function of the total shear rate under conditions for which a constant rate-of-strain is applied to one of the bounding walls. The square (labelled $\sigma_{y}$ ) indicates the static yield stress obtained by sequentially increasing the applied stress until the system begins to exhibit viscous $\mathrm{fl} \mathrm{w}$. For values of the stress between dynamic and static yield points, the system was found to exhibit inhomogeneous fow. (Reprinted with permission from [138]. Copyright 2004, American Institute of Physics.)

shear stress is plotted as a function of the total strain rate (which may differ from the local rate-of-strain) a dynamic yield stress can clearly be identifed. It was observed in the simulations that at suff ciently low (total) shear rates, an inhomogeneous fow profle develops in which a static layer coexists with a fuidized region exhibiting a linear fow prof le. In a complementary set of simulations a lower bound for the static yield stress was identif ed by slowly (stepwise) increasing the shear stress until viscous fl $\mathrm{w}$ could be detected at long times. The static yield stress thus obtained was found to provide a criterion for determining the onset of inhomogeneous fow. These observations may be consistent with experiments on PMMA colloids exhibiting inhomogeneous fow [136] but are apparently at odds with experiments on core-shell particles which do not give indications of banding or shear localization effects $[60,61,134]$. While these discrepancies remain to be understood, it seems possible that the softness of the potential interaction in the core-shell systems studied in $[60,61,134]$ may play a role in maintaining homogeneous fow.

\section{Theoretical approaches to flui states}

There currently exist several alternative theoretical approaches to frst-principles calculation of the microstructure and macroscopic theology of colloidal dispersions subject to extemally applied fow. Each of the available approximation schemes is tailored to capture the physically relevant aspects of the correlated particle motion within a restricted range of volume fractions. Theories aiming to treat low and intermediate volume fraction dispersions take as their common starting point the pair Smoluchowski equation, which is an exact coarse-grained reduction of the manybody Smoluchowski equation (14). At high volume fractions close to the glass transition the pair Smoluchowski equation no longer provides a convenient starting point and an alternative approach capable of capturing slow structural relaxation is required. This is provided by the recently developed integration through transients modecoupling theory $[16,17,20,135]$. In the following, we will $f$ rst introduce the pair Smoluchowski equation before proceeding to follow the "volume fraction axis" to give an overview of the current state of research on the theory of fowing states.

\subsection{The pair Smoluchowski equation}

While equation (14) provides a well def ned microscopic dynamics, it has been found useful to start from an equivalent coarse-grained level of description by integrating out unnecessary degrees of freedom from the outset. Assuming spatial translational invariance, integration of equation (14) over the centre-of-mass coordinate of a pair of particles and the remaining $N-2$ particles leads to an equation for the fow distorted pair correlation function as a function of $\mathbf{r}=\mathbf{r}_{2}-\mathbf{r}_{1}$ (see e.g. $[56,140-142])$

$$
\begin{aligned}
& \partial g(\mathbf{r})+\nabla_{r} \cdot\left[\mathbf{v}(\mathbf{r}) g(\mathbf{r})-\mathbf{D}(\mathbf{r}) \cdot \nabla_{r} g(\mathbf{r})\right] \\
& \quad \partial t \\
& =-\nabla_{r} \cdot[\mathbf{D}(\mathbf{r}) \cdot \beta \mathbf{F}(\mathbf{r}) g(\mathbf{r})]
\end{aligned}
$$

where we have suppressed explicit time-dependence in the function arguments for notational convenience and where we have introduced the gradient operator $\nabla_{r}=\nabla_{2}-\nabla_{1}$. The conditional probability to $\mathrm{f}$ nd particles at coordinates $\mathbf{r}_{3} \cdots \mathbf{r}_{\mathrm{N}}$, given that the frst two are known to be at locations $\mathbf{r}_{1}$ and $\mathbf{r}_{2}$, respectively, is given by $P\left(\mathbf{r}_{3}, \ldots, \mathbf{r}_{N} \mid \mathbf{r}_{1}, \mathbf{r}_{2}\right)=$ $P\left(\mathbf{r}_{1}, \ldots, \mathbf{r}_{N}\right) / P\left(\mathbf{r}_{1}, \mathbf{r}_{2}\right)$ and is required to calculate the functions $\mathbf{F}(\mathbf{r}), \mathbf{D}(\mathbf{r})$ and $\mathbf{v}(\mathbf{r})$ entering equation (24). The f rst of these functions, $\mathbf{F}(\mathbf{r})$, describes the force acting between our chosen pair of particles due to both direct potential interaction $v(r)$, taken here to be pairwise additive, and indirect interactions transmitted via the surrounding $N-2$ particles

$$
\begin{aligned}
& \mathbf{F}(\mathbf{r})=-\nabla_{r} v(r)-\frac{n}{2} \int \mathrm{d} \mathbf{r}_{3} \begin{array}{c}
g^{(3)}\left(\mathbf{r}_{1}, \mathbf{r}_{2}, \mathbf{r}_{3}\right) \\
g\left(\mathbf{r}_{1}, \mathbf{r}_{2}\right)
\end{array} \\
& \times\left(\nabla_{2} v\left(\left|\mathbf{r}_{2}-\mathbf{r}_{3}\right|\right)-\nabla_{1} v\left(\left|\mathbf{r}_{1}-\mathbf{r}_{3}\right|\right)\right),
\end{aligned}
$$

where $g^{(3)}\left(\mathbf{r}_{1}, \mathbf{r}_{2}, \mathbf{r}_{3}\right)$ is the nonequilibrium triplet distribution function. The diffusion tensor is similarly obtained by conditional averaging and contains details of the hydrodynamic interactions

$$
\mathbf{D}(\mathbf{r})=2 D_{0}\left({ }_{r^{2}}^{\mathbf{r r}} G(r)+\left(\delta-\begin{array}{r}
\mathbf{r r} \\
r^{2}
\end{array}\right) H(r)\right),
$$

where $\mathbf{r r}$ denotes a dyadic product and the scalar hydrodynamic functions $G(r)$ and $H(r)$ remain to be specif ed. Finally, the relative velocity of a pair of particles is given by

$$
\mathbf{v}(\mathbf{r})=\kappa \cdot \mathbf{r}+\mathbf{C}(\mathbf{r}): \bar{\kappa}
$$

where $\bar{\kappa}$ is the symmetric rate-of-strain tensor $\bar{\kappa}=(\kappa+$ $\left.\kappa^{\mathrm{T}}\right) / 2$ and $\mathrm{C}$ is the (third rank) hydrodynamic resistance tensor 
describing the disturbance of the aff ne fow due to the presence of the particles

$$
\mathbf{C}(\mathbf{r}): \bar{\kappa}=-r\left(\begin{array}{c}
\mathbf{r r} \cdot \overline{\boldsymbol{\kappa}} \cdot \mathbf{r} \\
r^{3}
\end{array} A(r)+\left(\boldsymbol{\delta}-{ }_{r^{2}}^{\mathbf{r r}}\right) \cdot{ }_{r}^{\overline{\boldsymbol{\kappa}} \cdot \mathbf{r}} B(r)\right) .
$$

The tensor $\mathbf{C}$ arises from purely geometrical considerations and is not material specif $\mathrm{c}$. It is interesting to note that the addition of hydrodynamic interactions prevents advection leading to unphysical hard-core overlap. A pair of approaching particles thus ' $\mathrm{fl} \mathrm{w}$ around' each other in the solvent fow, an effect taken care of by the second term in equation (27).

In the dilute limit, much is known about the hydrodynamic functions $A, B, G$ and $H$, as only an isolated pair of spheres must be considered. For both large and small separations analytical expressions for these functions exist [47] and are supplemented by tabulated numerical data for intermediate ranges [143]. At higher volume fractions approximations are required to obtain the hydrodynamic functions and a number of schemes have been developed which aim to incorporate the effects of many-body hydrodynamics [27, 49, 95, 144, 145]. In the absence of hydrodynamic interactions $A=B=0$ and $G=H=1$ leading to considerable simplif cation. It should be noted that $\mathbf{C}=0$ in this limit, with the consequence that affi e motion alone can lead to hard-core overlaps. While an exact treatment of the thermodynamic part of the problem would lend such unphysical conf gurations zero statistical weight, care must be exercised in approximate treatments which may satisfy only partially this important geometrical constraint.

Although the coarse-grained pair Smoluchowski equation (24) is still exact (under the assumption of homogeneity), it does not provide a closed expression for the microstructure, as encoded in $g(\mathbf{r})$. Evaluation of the integral term required to determine the force (25) demands knowledge of the nonequilibrium triplet distribution function, which remains unknown and contains the residual inf uence of the surrounding particles which have been integrated out. This situation is familiar from the BBGKY hierarchy [15] for which the triplet correlations must be approximated in terms of the pair correlations (using e.g. the Kirkwood superposition approximation) in order to arrive at a closed equation. In recent years, accurate approximations for the equilibrium triplet correlations of certain model systems have been developed [146, 147]. Less is known regarding the nature of the triplet correlations in nonequilibrium situations. Recent simulations [149] using accelerated Stokesian dynamics $[41,50]$ have revealed the existence of aligned particle triplets under shear and it may be hoped that such microstructural insights will eventually lead to improved theories by guiding the development of approximate closures for the triplet correlations. Some of the approaches to be reported in section 6.3 have attempted to make progress in this direction by approximating explicitly the integral term on the right-hand side of (24).

We note that, although the assumption of spatial homogeneity underlying (24) is mathematically convenient (for a translationally invariant system the only physically relevant coordinate is the separation vector $\mathbf{r}=\mathbf{r}_{2}-\mathbf{r}_{1}$ ), it may not be appropriate under all conditions. The presence

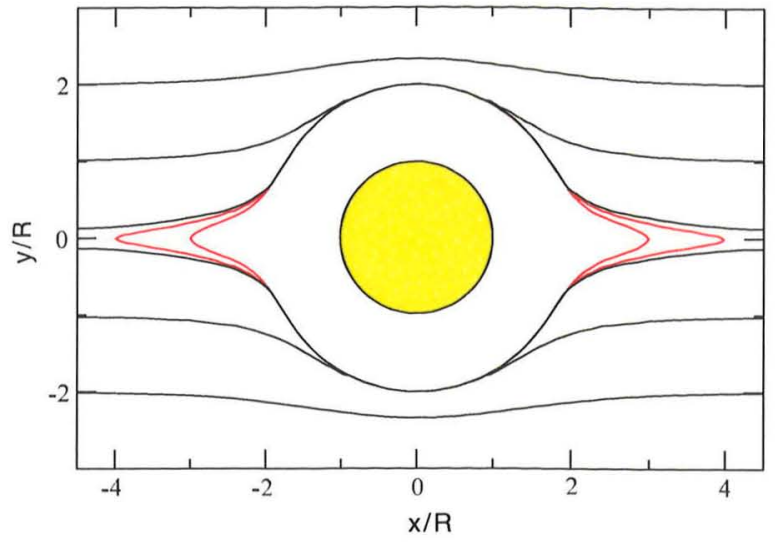

Figure 10. For mathematically perfect hard-spheres with a hydrodynamic radius equal to the radius of potential interaction, $R_{\mathrm{H}}=R$, particle pairs exhibit well define trajectories. Taking a reference frame in which one particle is fxed at the origin (yellow) the second particle (red) follows the trajectories shown in the 'pure hydrodynamic limit' of large $\mathrm{Pe}[30]$. The f gure shows some sample trajectories in the $z=0$ fow-gradient plane (see e.g. [6]). Closed orbits are indicated in red and open in black. The apparent fore-aft mirror symmetry gives rise to Newtonian rheology.

of spatial inhomogeneity induced by either external potential fie ds, shear banded or shear localized states complicates the coarse graining procedure, resulting in an inhomogeneous version of (24). While these issues should pose no diff culty at low volume fractions, for which the right-hand side of (24) may be disregarded, caution should be exercised when treating systems at higher volume fraction.

Once $g(\mathbf{r})$ is known, calculation of the stress tensor describing the macroscopic rheological response becomes possible. Although exact expressions relating the stress tensor to the fow distorted microstructure are known formally, the situation is complicated by the appearance of unknown conditionally averaged hydrodynamic functions in the expressions. However, reliable approximations for these functions are available and enable the stress to be evaluated directly from $g(\mathbf{r})$ [27]. For the simpler case of a system interacting via a pair potential and in the absence of hydrodynamic interactions, the stress tensor may be completely determined by a simple integral over the pair correlation function [148]

$$
\sigma=-n k_{\mathrm{B}} T 1+5 \eta_{\mathrm{s}} \phi \overline{\boldsymbol{\kappa}}-{ }_{2}^{n^{2}} \int \mathrm{d} \mathbf{r} \underset{r}{\mathbf{r r}} v^{\prime}(r) g(\mathbf{r}),
$$

where $v^{\prime}(r)$ is the derivative of the pair potential, $\eta_{\mathrm{s}}$ the solvent viscosity, 1 the identity matrix and $\mathbf{r r}$ denotes a dyadic product. Note that the second term on the right-hand side of equation (29) assumes that the particles possess a well def ned hard-core from which the solvent is excluded.

In the absence of fow the stress tensor is diagonal with the osmotic pressure given by $\Pi=-\operatorname{Tr} \sigma / 3$. For a system of pure hard-spheres the familiar equation of state $\beta \Pi / n=1+4 \phi g(d)$ is thus recovered. When under shear f ow equation (29) yields a shear viscosity due to the colloids $\eta=5 \phi \eta_{\mathrm{s}} / 2+\mathcal{O}\left(\phi^{2}\right)$, where the frst term corresponds to 


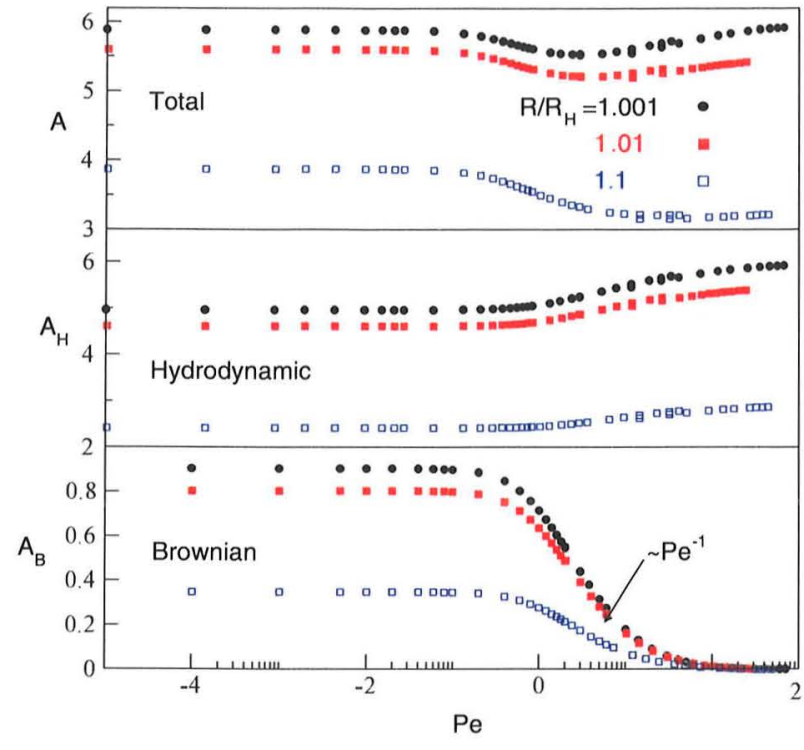

Figure 11. The pair contributions to the relative shear viscosity of a dilute colloidal dispersion under steady shear $\mathrm{fl} \mathrm{w}$ for three values of the ratio $R / R_{\mathrm{H}}$ (see figur 7). To second order in $\phi$ the relative viscosity is given by $\eta_{\mathrm{r}} \equiv \eta / \eta_{\mathrm{s}}=1+5 \phi_{\mathrm{H}} / 2+A\left(P e, R / R_{\mathrm{H}}\right) \phi^{2}$, where $\phi_{\mathrm{H}}=\left(R_{\mathrm{H}} / R\right)^{3} \phi$, and $A\left(P e, R / R_{\mathrm{H}}\right)$ can be divided into hydrodynamic and Brownian contributions $A=A_{\mathrm{H}}+A_{\mathrm{B}}$. As $R / R_{\mathrm{H}} \rightarrow 1$ the dispersion shows shear thickening at large $P e$ values due to the increase in the hydrodynamic contribution to the shear stress. As the value of $R / R_{\mathrm{H}}$ is increased the increase in $A_{\mathrm{H}}$ becomes balanced by the decrease in $A_{\mathrm{B}}$ and only shear thinning remains. (Figure adapted from [133].)

Einstein's classic dilute limit result [150] and the corrections to higher order in $\phi$ come from the anisotropy of $g(\mathbf{r})$ inside the integral term. It is clear from equation (29) that fow induced microstructural anisotropy can give rise to the f nite normal stress differences $N_{1}=\sigma_{\mathrm{xx}}-\sigma_{\mathrm{yy}}$ and $N_{2}=\sigma_{\mathrm{yy}}-\sigma_{\mathrm{zz}}$ characteristic of non-Newtonian rheology. The dyadic weight factor entering the integral term has the consequence that if $g(\mathbf{r})$ possesses a mirror symmetry about the $x=0$ plane then the integral term will be equal to zero and the rheology will thus be Newtonian. While this 'fore-aft' symmetry of the pair distribution function is an exact mathematical consequence of the 'pure hydrodynamic limit', in which the motion of the particles is determined by Stokes fow alone [151, 152], chaotic many-body particle motion and experimental perturbations, such as particle surface roughness, present in real colloidal systems break the symmetry and result in a non-Newtonian rheology [56].

\subsection{Low volume fraction}

Efforts to obtain a microscopic understanding of colloid rheology began with the seminal 1906 work of Einstein in which it was shown how the shear viscosity of a dilute dispersion of hard spherical colloids increases with colloidal volume fraction, assuming that both the volume fraction and the shear rate remain small $\left(\eta=\eta_{\mathrm{s}}(1+5 \phi / 2)\right)$ [150]. Einstein's study addressed the one-body problem of a single colloid suspended in a Newtonian fuid. The next step is naturally to consider the interaction between pairs of colloidal particles, thus making possible a discussion of the pair correlation functions and their relation to rheological functions at low volume fraction. Study of the two-particle dilute limit was initiated by Batchelor [30, 151-153] whose fundamental work formed the basis for the more recent investigations by Brady and co-workers [56, 133, 154].

At low volume fraction, equation (24) admits analytical solution in the limits $P e \rightarrow 0$ and $\infty[56,154]$ and precise numerical results have been obtained for intermediate values of $P e$, both with and without hydrodynamic interactions [133, 157]. Exact results are made possible by the fact that, in the dilute limit, triplet correlations in the pair Smoluchowski equation may be neglected leading to a closed expression for $g(\mathbf{r})$. Neglecting the diff cult integral term in equation (24) incurs an $\mathcal{O}(\phi)$ error which becomes irrelevant as $\phi \rightarrow 0$ and yields a closed equation for $g(\mathbf{r})$ which is exact to lowest order in the volume fraction and valid for all $P e$ values. For the simple special case of hard-spheres equation (24) thus reduces to the equation-of-motion

$$
\frac{\partial g(\mathbf{r})}{\partial t}+\nabla_{r} \cdot\left[\mathbf{v}(\mathbf{r}) g(\mathbf{r})-P e^{-1} \boldsymbol{D}(\mathbf{r}) \cdot \nabla g(\mathbf{r})\right]=0,
$$

where we have scaled distance and time with particle radius and fow rate, respectively, such that $P e$ appears explicitly. In order to fully specify the problem equation (30) must be supplemented with appropriate boundary conditions enforcing both the requirement that the particles do not penetrate, via a no-f ux condition at $r=d$, and that $g(\mathbf{r}) \rightarrow 1$ as $r \rightarrow \infty$ [6]. The frst of these boundary conditions is clearly an exact physical requirement and is valid also at higher volume fractions. The second condition assumes the decay of 'wake' structures which develop in $g(\mathbf{r})$ downstream from the reference particle at higher fow rates. Detailed analysis of (30) has shown that the range of the wake scales linearly with $P e$, thus justifying the choice of boundary conditions.

For systems interacting via a spherically symmetric pair potential it can be shown that, regardless of volume fraction, in the weak fow limit $P e \rightarrow 0$ a steady fow fied acts on the spherically symmetric equilibrium distribution $g_{\text {eq }}(r)$ to produce an $\mathcal{O}(\mathrm{Pe})$ perturbation $[6,153]$

$$
g(\mathbf{r})=g_{\mathrm{eq}}(r)\left[1-P e^{\mathbf{r} \cdot \hat{\boldsymbol{\kappa}} \cdot \mathbf{r}} f(r)\right],
$$

where $\hat{\kappa}=\bar{\kappa} / \sqrt{ } 2 \bar{\kappa}: \bar{\kappa}$, with $\bar{\kappa}$ is def ned below equation (29). In the dilute limit $g_{\text {eq }}(r)=\Theta(-r-1)$ and substitution of (31) into (30) yields a differential equation for the dimensionless function $f(r)$ which has been solved for several interaction potentials of interest [6]. Although analytical expressions exist for certain systems, a numerical integration is still required to obtain $f(r)$ in the special case of hard-spheres with hydrodynamic interactions $[6,153]$.

The extension of equation (31) to higher order in the Peclet number has been analysed in considerable detail [154]. It can be shown that $g(\mathbf{r})$ has a regular perturbation expansion to $\mathcal{O}\left(P e^{2}\right)$ but that calculation of the next order term requires singular perturbation theory, yielding an $\mathcal{O}\left(P e^{5 / 2}\right)$ correction. 
The calculation of higher order terms in the $P e$ expansion requires use of matched asymptotic expansions which rapidly become intractable and make preferable a numerical solution of $(30)$. The expansion of the distorted structure is given by $[154]$

$$
g(\mathbf{r})=1+f_{1} P e+f_{2} P e^{2}+f_{5 / 2} P e^{5 / 2}+\cdots,
$$

where comparison with (31), and noting that $g_{\mathrm{eq}}(r>2 R)=1$ for hard-spheres at low volume fraction, enables identif cation of the coeff cient $f_{1}$. To $\mathcal{O}(P e)$ the rheology is predicted to be Newtonian with normal stress differences identically equal to zero [30]. Non-Newtonian rheology frst occurs at $\mathcal{O}\left(P e^{2}\right)$, which is suff cient to capture both nonzero normal stresses and the frst fow induced correction to the osmotic pressure [154].

Analytic solutions to equation (30) exist also in the 'pure hydrodynamic limit' of strong fows $(\mathrm{Pe} \rightarrow$ $\infty)$ and have highlighted the subtle balance between hydrodynamic and potential forces in determining the rheological response [30, 152]. For large Peclet number steady fows the solution of equation (30) is well approximated by the solution of the simplif ed equation $\nabla \cdot[\mathbf{v}(\mathbf{r}) g(\mathbf{r})]=0$ (subject to the boundary condition $g(\mathbf{r})=1$ at $r=\infty$ ), despite the fact that the approximation neglects the boundary layer and thus violates the no-fux condition at contact. Subject to certain conditions on the trajectories of particle pairs, Batchelor and Green proved the surprising result that the simplif ed equation predicts a spherically symmetric radial distribution function for hard-spheres, leading to Newtonian rheology [152]. This clear prediction is a direct consequence of the fore-aft symmetry of $g(\mathbf{r})$ (see section 6.1) inherent in the assumed Stokesian solvent fow. In f gure 10 we show sample trajectories of a hard-sphere in shear fow as it moves around a second sphere held fxed at the coordinate origin. Of particular interest is the existence of closed trajectories along which the particles become trapped in bound orbits and which are connected to the lubrication force required to displace solvent from the region between the particles [47]. In fact, the lubrication force acting between a pair of perfect spheres at separation $r$ shows a divergence, $F_{\text {lub }} \sim(r / R-2)^{-1}$, corresponding to surface contact. Crucially, the time-reversibility of the Stokes equations dictating the solvent fow implies that the force required to push particles together is identical to that required to pull them apart, with the consequence that particle trajectories exhibit the fore-aft mirror symmetry apparent in f gure 10.

Despite the sound mathematical evidence provided in [152], serious doubts were cast by subsequent experiments on intermediate volume fraction hard-sphere-like colloidal dispersions, which seemed to contradict the theoretical predictions by identifying a non-Newtonian rheology at large fl $w$ rates [155]. It should be noted that the reversibility of Stokes fow implies that fore-aft symmetry in the pure hydrodynamic limit holds also for $f$ nite volume fractions and so the value $\phi=0.4$ employed in the experiments of [155] cannot be held responsible for the apparent discrepancy. The situation was eventually resolved by Brady and Morris, who analytically identif ed a boundary layer in the region close to particle contact in which Brownian motion balances advection [56]. The analysis of [56] indeed recovers the fndings of [152] in the case that the hydrodynamic radius is equal to the excluded volume radius, as would be the case for mathematically perfect hard-spheres with no surface roughness. However, when the excluded volume radius exceeds the hydrodynamic radius, even by a very small amount, the residual Brownian motion within the anisotropic boundary layer of $g(\mathbf{r})$ leads to a non-Newtonian rheology in the strong fow limit. Figure 7 shows a sketch of the model employed in [56]. The physical origin of these symmetry breaking surface effects remains an open problem and is likely to be a function of various system specifi parameters (e.g. surface roughness). We note that the existence of a boundary layer structure was originally identif ed in studies of the distorted structure factor of colloids under shear [156].

The analytical results for $\mathrm{Pe} \rightarrow 0$ and $\infty$ for hard-spheres under steady shear have been both conf rmed and supplemented by full numerical solutions at all values of $P e[133,157]$. These accurate numerical studies revealed that the dilute dispersions described by equation (30) demonstrate not only shear thinning and f nite normal stresses at intermediate $\mathrm{fl} \mathrm{w}$ rates (accessible from the expansion (32) to $\mathcal{O}\left(P e^{2}\right)$ ), but also shear thickening at high fow rates [133]. Shear thinning in dilute systems is correlated with a nonvanishing distortion of the structure factor in the plane perpendicular to the fow direction [158].

In fgure 11 we show some of the numerical results obtained in [133] for the shear viscosity of a dilute dispersion as a function of $P e$. In these calculations the effective sphere model sketched in f gure 7 was employed and results are shown for three values of the ratio of potential to hydrodynamic radius, $R / R_{\mathrm{H}}$. To second order in $\phi$ the relative viscosity may be expressed as

$$
\eta_{r} \equiv \eta / \eta_{\mathrm{s}}=1+5 \phi_{\mathrm{H}} / 2+A\left(P e, R / R_{\mathrm{H}}\right) \phi^{2},
$$

where $\phi_{\mathrm{H}}=\left(R_{\mathrm{H}} / R\right)^{3} \phi$ is the volume fraction with respect to the hydrodynamic radius and the function $A\left(P e, R / R_{\mathrm{H}}\right)$ contains the effects of microstructural distortion (note that calculation of $g(\mathbf{r})$ to $\mathcal{O}(\phi)$ yields the viscosity to $\mathcal{O}\left(\phi^{2}\right)$ ). The numerically determined function $A\left(P e, R / R_{\mathrm{H}}\right)$ may be further split into hydrodynamic and Brownian contributions, $A=A_{\mathrm{H}}+A_{\mathrm{B}}$, which are independently accessible from the numerical calculations of [133].

The top panel of $f$ gure 11 shows $A$ as a function of $P e$. For shear rates up to $P e \sim 1$ the qualitative variation of the viscosity is independent of the value of the size ratio $R / R_{\mathrm{H}}$, displaying a low shear Newtonian plateau followed by shear thinning. The $R / R_{\mathrm{H}}$ independence of the form of the curves for $P e<1$ ref ects the fact that hydrodynamic interactions are not central to the mechanisms underlying shear thinning and only infuence the absolute value of the viscosity. For $P e>1$ the viscosity begins to increase as a function of $P e$ and the dispersion shear thickens. In contrast to the shear thinning behaviour, the viscosity increase is strongly sensitive to the value of $R / R_{\mathrm{H}}$. In the limit $R / R_{\mathrm{H}} \rightarrow 1$ the trajectories sketched in fi ure 10 are recovered and Batchelor's pure hydrodynamic limit is realized with a viscosity independent of $P e$. In [133] it is also shown that for $R / R_{\mathrm{H}} \rightarrow 1$ the normal 
stresses also vanish as $P e \rightarrow \infty$, indicating a Newtonian response. As $R / R_{\mathrm{H}}$ is increased the magnitude of the shear thickening reduces strongly and by $R / R_{\mathrm{H}}=1.1$ is lost entirely. This trend strongly indicates the important inf uence of short range lubrication forces on shear thickening (see section 5.3), which can effectively be turned-off by slightly reducing the hydrodynamic radius below that of the repulsive potential interaction.

The central and lower panels of fi ure 11 show the individual hydrodynamic and Brownian pair contributions to the total stress as a function of $P e$. For $P e<1$ it is apparent that the shear thinning is due to a reduction in the Brownian contribution with increasing $P e$. For values of $R / R_{\mathrm{H}}$ close to unity the reduction in $A_{B}$ is more than compensated by an increase in the hydrodynamic stress afor $P e>1$, leading to shear thickening. However, increasing $R / R_{\mathrm{H}}$ above unity rapidly suppresses the inf uence of lubrication and the hydrodynamic contribution is overwhelmed by the strong drop in $A_{\mathrm{B}}$.

Comparing the frst panel of fgure 11 with f gure 4 , it is remarkable the extent to which the qualitative rheological response observed in systems at fnite volume fraction is reproduced by calculations based on the dilute limit. However, the fact that $W i=P e$ at low volume fractions does not permit investigation of potentially interesting interaction effects between shear thinning and thickening. Despite the extensive understanding of the response of dilute dispersions to steady fows, analogous solutions for time-dependent fows remain to be investigated. This leaves open many interesting questions regarding transient response and nonsteady states.

\subsection{Intermediate volume fraction}

The simplest way to extend the dilute limit results to f nite volume fraction is via the introduction of empirical volume fraction dependent scale factors $[56,145,154,160]$. Analysis of equation (30) has provided two important insights. Firstly, at f nite volume fractions the value of $g(\mathbf{r})$ outside the boundary layer close to the surface of a reference particle should asymptote to the solution of $\nabla \cdot[\mathbf{v}(\mathbf{r}) g(\mathbf{r})]=0$, for which the no-f ux boundary condition is ignored. This amounts to assuming that, outside the thin boundary layer, it is suff cient to solve a purely advective problem. Secondly, that the appropriate Peclet number in the presence of hydrodynamics is $P e_{\mathrm{H}}=\dot{\gamma} R^{2} / 2 D_{s}(\phi)$, where $D_{s}(\phi)$ is the short-time diffusion coeffic ent (which differs from $D_{0}$ due to hydrodynamic interactions with neighbouring particles). For weak fows $(P e \ll 1)$ these ideas are manifest in a modifie perturbation to the quiescent pair correlations

$$
g(\mathbf{r})=g_{\mathrm{eq}}(r)\left[1-P e_{\mathrm{H}} \begin{array}{c}
\mathbf{r} \cdot \hat{\kappa} \cdot \mathbf{r} \\
r^{2}
\end{array}\right.
$$

The f nite volume fraction equilibrium radial distribution function $g_{\mathrm{eq}}(r)$ is an external input to the theory and can be calculated using either simulation or equilibrium integral equation theory [15]. The function $f(r)$ is determined by substitution of (34) into the dilute limit equation (30), or its generalization for non-hard-sphere potentials. Many-body effects are thus included via the equilibrium radial distribution function and the short-time diffusion coeff cient entering $P e_{\mathrm{H}}$. It should be noted that Brady's approach assumes an input $g_{\text {eq }}(r)$ which diverges at random-close-packing $(\phi \approx 0.64)$.

Despite considerable success, the scaling approach suffers from two significa $t$ drawbacks. (i) The mathematical structure of the nonequilibrium part of the theory is that of the dilute system. Thus, regardless of rescaling, this approach does not admit the occurrence of possible additional physical mechanisms which may only occur as a consequence of cooperative behaviour at fnite volume fraction. (ii) The equilibrium microstructure $g_{\mathrm{eq}}(r)$ is required as an external input and does not emerge from an approximate treatment of many-body correlation effects within the theory. These issues may be addressed by approaches which aim to approximate the triplet distribution function entering the pair Smoluchowski equation (24) via the effective force between a pair of particles (25). In order to arrive at a closed theory it is necessary to relate $g^{(3)}\left(\mathbf{r}_{1}, \mathbf{r}_{2}, \mathbf{r}_{3}\right)$, either explicitly or implicitly (by approximating weighted integrals over the triplet distribution), to $g(\mathbf{r})$ and the pair potential $v(r)$ using an appropriate 'closure' hypothesis.

6.3.1. Superposition approximation. Guidance in developing an appropriate closure relation to treat nonequilibrium states is provided by experience from equilibrium liquid state integral equation theory. One of the earliest approximation schemes aiming to arrive at a closed equation for the equilibrium pair correlations was developed by Born and Green [161] who employed the Kirkwood superposition approximation $g^{(3)}\left(\mathbf{r}_{1}, \mathbf{r}_{2}, \mathbf{r}_{3}\right) \approx g\left(r_{12}\right) g\left(r_{23}\right) g\left(r_{13}\right)$ in combination with the second member of the exact Yvon-Born-Green hierarchy (equation (37)) $[15,162]$. Numerical solution of the resulting Born-Green equation yields acceptable results only for weak coupling. The superposition approximation is asymptotically correct for large particle separations but is poor when particles come close to contact, leading to a failure of the BornGreen equation at intermediate volume fractions (a breakdown which was erroneously taken as an indicator for the frst order freezing transition of hard-spheres). Subsequent attempts have aimed to systematically improve upon the superposition approximation by including additional Mayer cluster diagrams (see e.g. [163]).

One of the earliest attempts to close the nonequilibrium equation (24) using the superposition approximation, albeit in the absence of hydrodynamics, was made by Ohtsuki [164] (self-diffusion was addressed using an analogous approach in [165]). Numerical solution of the closed integro-differential equation resulting from this approximation was performed for charged hard-spheres at intermediate volume fraction. Although the theoretical results for the zero-shear viscosity were found to be in reasonable agreement with those of experiment, the pair correlation $g(\mathbf{r})$ was found to be in considerable error. These f ndings are supported by the work of Wagner and Russel who investigated a similar superposition based approach [140].

The observed discrepancies in $g(\mathbf{r})$ arising from superposition are not surprising: in equilibrium, the pair $f u x$ in 
equation (24) may be set equal to zero, resulting in an exact equation for the pair correlation function

$$
k_{\mathrm{B}} T \nabla_{r} \ln g_{\mathrm{eq}}(r)=\mathbf{F}(\mathbf{r})
$$

where $\mathbf{F}(\mathbf{r})$ is given by equation (25) and contains the unknown triplet distribution function. Use of superposition to approximate $g^{(3)}\left(\mathbf{r}_{1}, \mathbf{r}_{2}, \mathbf{r}_{3}\right)$ in equation (25) leads directly to the Born-Green equation for the equilibrium correlations, the shortcomings of which have been noted above. It is therefore nontrivial that, despite a relatively poor description of the microstructure, the results for the zero-shear viscosity presented in [164] turn out to be rather good agreement with experimental data. A similar situation is encountered in a number of the theories to be outlined in this section and serves to highlight the fact that good values for integrated quantities (e.g. the viscosity) do not necessarily imply that the underlying correlations are treated adequately.

When applying superposition to tackle nonequilibrium problems it should be borne in mind that the approximation represents an uncontrolled ansatz and only possesses a frm statistical mechanical basis in the limit of vanishing fow rate. In equilibrium, the superposition approximation represents both the exact low volume fraction limit of the triplet correlation function and recovers correctly the long range asymptotic behaviour. Analogous limiting results for the nonequilibrium triplet correlations which could motivate a more appropriate superposition-type approximation are currently lacking and would require a detailed analysis of the triplet Smoluchowski equation in the dilute limit. Despite these shortcomings, superposition may nevertheless turn out to be useful for some applications. As noted at the end of section 5.2, very recent simulation results investigating the microstructure of hard-sphere f uids under shear fow revealed the importance of linear triplet conf gurations [104]. From equilibrium studies it is known that the error of the superposition approximation is reduced for such linear conf gurations [166], thus raising the interesting possibility that superposition may be appropriate for treating certain nonequilibrium states.

6.3.2. Potential of mean force. Inspection of the exact expression for the force (25) shows that only a certain weighted integral of the triplet distribution is required to determine the pair correlations. It is thus not strictly necessary to know the full details of the triplet distribution and schemes can be developed which aim to approximate directly the integrated quantity. The simplest approximation possible is to neglect entirely the integral contribution to the effective force (25) and set

$$
\mathbf{F}(\mathbf{r})=-\nabla_{r} v(r) .
$$

Combining this crude approximation with equation (24) recovers the dilute limit equation-of-motion for $g(\mathbf{r})$ for an arbitrary spherically symmetric pair potential $v(r)$. For the special case of hard-spheres this leads to equation (30), but with an additional delta function term on the right-hand side. As demonstrated by Cichocki [167] the resulting equation is completely equivalent to (30), with zero right-hand side, supplemented by a no-f ux boundary condition at contact.
As already noted, in equilibrium the pair Smoluchowski equation (24) reduces to the Yvon-Born-Green equation for the pair correlations

$$
\begin{aligned}
k_{\mathrm{B}} T & \nabla_{r} \ln g_{\mathrm{eq}}(\mathbf{r})=-\nabla_{r} v(r)-\frac{n}{2} \int \mathrm{d} \mathbf{r}_{3} g_{\mathrm{eq}}^{(3)}\left(\mathbf{r}_{1}, \mathbf{r}_{2}, \mathbf{r}_{3}\right) \\
& \quad \times\left(\nabla_{2} v\left(\left|\mathbf{r}_{2}-\mathbf{r}_{1}\right| \mathbf{r}_{2}\right)-\nabla_{1} v\left(\left|\mathbf{r}_{1}-\mathbf{r}_{3}\right|\right)\right) \\
\equiv & -\nabla v_{\mathrm{mf}}(\mathbf{r}),
\end{aligned}
$$

where $v_{\mathrm{mf}}(\mathbf{r})$ is the equilibrium potential of mean force [15], def ned by $g_{\text {eq }}=\exp \left(-\beta v_{\mathrm{mf}}\right)$ in analogy with the low density limit of the pair correlations. A frst step towards improving the zeroth order approximation (36) is thus to approximate the nonequilibrium force (25) by the equilibrium potential of mean force, leading to

$$
\mathbf{F}(\mathbf{r})=-k_{\mathrm{B}} T \nabla_{r} \ln g_{\mathrm{eq}}(r) .
$$

This approximation, developed by Russel and Gast [141], incorporates equilibrium thermodynamic many-body couplings but, as is clear from equation (37), neglects the inf uence of fow on the triplet correlations. It should be noted that the approximation (38) does not provide any information regarding the nonequilibrium triplet correlation function. This is in contrast to superposition based approaches from which the triplet function can be reconstructed using a product of the self-consistently determined pair correlation functions. It should also be noted that within the Russel-Gast approach the function $g_{\mathrm{eq}}(r)$ is an input, which can be calculated using either simulation or equilibrium statistical mechanical approximations (see section 6.3.3).

The linear equation resulting from combining equations (24) and (38) has been solved for hard-spheres in weak shear fl $\mathrm{w}[141]$. In these calculations, simple approximations were employed to determine the hydrodynamic functions $A, B, G$ and $H$ entering equations (26) and (28) def ning the conditionally averaged hydrodynamic tensors which represent the effective medium (solvent $+(N-2)$ colloids) in which the chosen pair of particles are immersed. Results were obtained for the zero-shear viscosity, linear response moduli $G^{\prime}(\omega), G^{\prime \prime}(\omega)$ under small amplitude oscillatory shear and the leading order fl $\mathrm{w}$ induced distortion of $g(\mathbf{r})$ (via determination of the function $f(r)$, see equation (31)). For $\phi<0.3$ good agreement with experiment was obtained for the integrated quantities $\eta_{0}, G^{\prime}$ and $G^{\prime \prime}$, despite providing only a poor description of the microstructure (see f gure 6 in [168]).

An interesting feature of the Russel-Gast theory is that the predicted zero-shear viscosity becomes very large in the vicinity of random-close-packing $(\phi \approx 0.64)$, although the precise nature of this rapid increase as a function of volume fraction remains to be studied in detail. The apparent divergence of $\eta_{0}$ is a nontrivial output of the theory, given that the approximate Verlet-Weiss expression for $g_{\mathrm{eq}}(r)$ used as input diverges only at $\phi=1.0$ [169]. When viewed within the context of the time correlation/Green-Kubo formalism (see section 5.1) it is tempting to infer that the observed growth in $\eta_{0}$ is related to the development of an underlying slow structural relaxation time. The solution of equation $(38)$ for small amplitude oscillatory shear at fnite frequencies would enable this issue to be addressed. 
6.3.3. Equilibrium integral equations. The Russel-Gast theory outlined above neglects the infuence of external fow on the triplet correlation function, which leads to a force $F(r)$ generated from the equilibrium potential of mean force (equation (38)). In order to go beyond this closeto-equilibrium ansatz it is necessary to express the force $\mathbf{F}(\mathbf{r})$ as a functional of $g(\mathbf{r})$, such that both functions can be determined self-consistently. A promising approach in this direction is to generalize equilibrium liquid state integral equation theory $[15]$ to treat the nonequilibrium problem. It will thus be useful to review brief y some concepts from the equilibrium theory before moving on to more unfamiliar territory in section 6.3 .4 .

Integral equation theories generally aim to calculate the equilibrium pair correlation function $g_{\mathrm{eq}}(r)$ from knowledge of the interaction potential in a non-perturbative fashion. Fundamental to the integral equation approach is the OrnsteinZernike (OZ) equation which, for a translationally invariant system, is given by the convolution form [15]

$$
h_{\mathrm{eq}}\left(r_{12}\right)=c_{\mathrm{eq}}\left(r_{12}\right)+n \int \mathrm{d} \mathbf{r}_{3} c_{\mathrm{eq}}\left(r_{13}\right) h_{\mathrm{eq}}\left(r_{32}\right)
$$

where $h_{\mathrm{eq}}(r)=g_{\mathrm{eq}}(r)-1$. The direct correlation function $c_{\text {eq }}(r)$ defned by (39) is a function of simpler structure than $h_{\mathrm{eq}}(r)$ and is thus easier to approximate. When supplemented by an independent closure relation between $c_{\mathrm{eq}}(r)$ and $h_{\mathrm{eq}}(r)$, containing details of the interaction potential under consideration, (39) provides a closed equation for the pair correlations (note that for pairwise additive potentials the triplet distribution does not enter explicitly). The task of the integral equation practitioner is thus to f nd numerically tractable closures which capture the essential physics of the problem under consideration.

Using diagrammatic techniques [170] it can be shown that a formally exact closure relation is given by

$$
g_{\mathrm{eq}}(r)=\exp \left[-\beta v(r)+h_{\mathrm{eq}}(r)-c_{\mathrm{eq}}(r)+b_{\mathrm{eq}}(r)\right],
$$

where $b_{\text {eq }}(r)$ is the unknown 'bridge function" containing the diff cult to evaluate 'irreducible' Mayer cluster diagrams [15]. Two closures of particular merit are the hypernetted-chain (HNC) and Percus-Yevick (PY), given by

$$
\begin{array}{r}
b_{\mathrm{eq}}=0 \quad(\mathrm{HNC}) \\
b_{\mathrm{eq}}=-\ln \left(1+h_{\mathrm{eq}}-c_{\mathrm{eq}}\right)-\left(h_{\mathrm{eq}}-c_{\mathrm{eq}}\right)
\end{array}
$$

respectively. An additional practical advantage of theories based on (39) over superposition-type approaches is that the convolution form of the integral term enables self-consistent solutions to be obtained using eff cient iterative numerical algorithms [171].

Although the majority of integral equation theories focus on the pair correlations, triplet correlations can also be handled within the same framework [147]. In addition to providing a higher level of resolution, the development of tripletlevel integral equations is motivated by the desire for an improved description of the pair correlations. Exact relations, such as the YBG equation (37), connect the triplet to the pair correlations and the expectation is that errors in an approximate $g_{\mathrm{eq}}^{(3)}$ may be averaged out by integration to the pair level. During the mid-1960s many of the leading liquid state theorists proposed integral equations for the triplet correlations (e.g. Verlet [172-176], Wertheim [177], Baxter [178], Stell [179]), all of which showed considerable promise. However, the complexity of solving the equations has hindered progress along this route and somewhat simpler, numerically tractable, theories now seem preferable [146, 147, 180].

An integral equation which will be of particular relevance for section 6.3 .4 was derived by Scherwinski [181]. Within the Scherwinski approximation the triplet correlation function is obtained from self-consistent solution of the following linear equation

$$
\begin{aligned}
& g_{\mathrm{eq}}\left(\mathbf{r}_{1}, \mathbf{r}_{2}, \mathbf{r}_{3}\right)=g_{\mathrm{eq}}\left(r_{12}\right) g_{\mathrm{eq}}\left(r_{13}\right) g_{\mathrm{eq}}\left(r_{23}\right)+n g_{\mathrm{eq}}\left(r_{12}\right) \\
& \quad \times \int \mathrm{d} \mathbf{r}_{4}\left(\begin{array}{c}
g_{\mathrm{eq}}^{(3)}\left(\mathbf{r}_{1}, \mathbf{r}_{3}, \mathbf{r}_{4}\right) \\
g_{\mathrm{eq}}\left(r_{14}\right)
\end{array}-g_{\mathrm{eq}}\left(r_{13}\right)\right) h_{\mathrm{eq}}\left(r_{14}\right) h_{\mathrm{eq}}\left(r_{24}\right) .
\end{aligned}
$$

Iteration of equation (43) yields an inf nite series expressing the triplet correlation function as a functional of the pair correlation function $g_{\text {eq }}(r)$. Substitution of this series into the exact YBG equation (37) yields a diagrammatic expansion for $g_{\mathrm{eq}}(r)$ in perfect agreement with that arising from solution of equations $(39)-(41)$. In this sense, equation (43) represents a triplet generalization of the more familiar pair-level HNC theory. The more powerful approximations proposed by earlier workers [172-179] probably provide a superior description of the triplet correlations than equation (43) and would, upon substitution into the YBG equation, lead to improved (i.e. better than standard $\mathrm{HNC}$ ) estimate of the pair correlations. However, the Scherwinski approximation has a number of purely technical advantages which make it particularly suitable for application to nonequilibrium situations and which are convenient for numerical implementation (see also [140], which predates [181], but contains several of the key ideas).

\subsubsection{Nonequilibrium integral equations. In order to} go beyond the Russel-Gast approximation [141] outlined in section 6.3.2, Lionberger and Russel employed the Scherwinski equation for the triplet correlation function (43) in order to estimate the force (25) entering the pair Smoluchowski equation [182]. Nonequilibrium pair and triplet correlations are thus determined self-consistently and are both infuenced by the externally imposed fow. It should be noted that the direct application of an equilibrium relation, such as equation (43), to nonequilibrium ignores the fact that nonequilibrium states are intrinsically different from equilibrium and thus represents a major approximation.

The original version of the Lionberger-Russel theory presented in [182] neglected hydrodynamic interactions and has been implemented numerically for weak fl ws only. No results beyond leading order in $P e$ have been presented, although in principle the theory remains valid also in the nonlinear regime. For $\phi<0.4$ the LR theory makes predictions for the zero-shear viscosity, selfdiffusion coeff cient and distorted microstructure in reasonable 
agreement with available computer simulation results. For $\phi \geqslant$ 0.45 signif cant quantitative deviations appear and the theory becomes unreliable. We note that the study [182] considered a suspension interacting via a continuous repulsive potential which was then mapped onto a hard-sphere system. The input equilibrium microstructure was generated using the RogersYoung integral equation [183] (an interpolation between PY and $\mathrm{HNC}$ ), despite the fact that the $P e \rightarrow 0$ limit of the theory reduces to the HNC approximation for $g_{\text {eq }}(r)$.

Although the Lionberger-Russel theory [182] provides a sophisticated treatment of the microstructural distortion by incorporating the triplet correlations into the self-consistency loop, it is interesting that the results for $\eta_{0}$ are inferior to those from the much simpler Russel-Gast theory [141] at high volume fractions. In particular, the former predicts only a relatively weak growth of $\eta_{0}$ with volume fraction, whereas the latter suggests a divergence. Calculations of the linear moduli $G^{\prime}, G^{\prime \prime}$ as a function of frequency have been performed using the LR theory and, perplexingly, reveal a structural relaxation time which decreases with increasing volume fraction. This unphysical prediction would appear to be at odds with the growth of $\eta_{0}$ output from the theory and represents a weak point of the approach, in need of clarif cation [184]. We note that the only approximation invoked by the Lionberger-Russel theory is the Scherwinski closure (43) for the triplet correlation function. It would therefore be interesting, albeit numerically demanding, to see whether any of the more sophisticated triplet closures available $[146,172-180]$ can improve the performance at higher volume fractions.

The theory developed in [182] omitted hydrodynamic interactions in order to make clearer the approximations to the many-body thermodynamic couplings and to facilitate comparison with Brownian dynamics simulation results. In [184] hydrodynamic interactions were included into the theory of [182] in order to make closer contact with experiment. The hydrodynamic approximations developed were found to be of the correct magnitude but errors in the underlying thermodynamic approximation, namely the Scherwinski equation (43), led to an underestimation of the magnitude of the nonequilibrium structure. It was identif ed that the magnitude of the fow induced structural distortion is determined by the slow structural relaxation in the system (a f nding supported by subsequent theoretical studies [185]), which, taken together with the results for the volume fraction dependence of the linear response moduli, suggests that the integral equation approach does not capture the slow dynamics characteristic of dense systems.

Wagner and Russel [140] developed an integral equation approach closely related to that of Lionberger and Russel in which the nonequilibrium triplet correlations are approximated using a closure motivated by the PY equilibrium theory (42). Although use of a PY-type closure ensures that excluded volume packing constraints are treated realistically, at least close-to-equilibrium, the theory of [140] included hydrodynamic interactions by employing only the low density limit of the pair hydrodynamic functions $A, B, G$ and $H$ entering equations (26) and (28). The simultaneous introduction of hydrodynamic and thermodynamic approximations in [140] served to obscure the validity of the proposed nonequilibrium PY approximation.

When using integral equation methods to tackle the triplet correlations in nonequilibrium it is important to bear in mind that the physical situation is intrinsically different from that in equilibrium. Consequently, caution must be exercised when attempting to apply trusted and familiar results from equilibrium statistical mechanics to a system under fow. In order to appreciate more clearly the approximations involved in applying equilibrium triplet closures to the pair Smoluchowski equation both Lionberger and Russel [182] and Szamel [168] have invoked the concept of a 'f ctitious' fow dependent twobody potential $u(r, \dot{\gamma})$. In a study of the kinetic theory of hardspheres Resibois and Lebowitz [186] assumed the existence of a two-body potential $u(\mathbf{r}, \dot{\gamma})$ which, if employed in an equilibrium calculation, reproduces exactly the nonequilibrium pair correlation function

$$
g(\mathbf{r})=g_{\mathrm{eq}}(\mathbf{r} ;[u]),
$$

where the square brackets indicate a functional dependence and where $g_{e q}$ is anisotropic as a result of the anisotropy of $u$. The $f$ ctitious potential thus serves as proxy for the fow f eld acting on the real system. It should be made clear that the assumption that an effective two-body potential can yield the correct $g(\mathbf{r})$ is quite distinct from the (erroneous) assumption that an effective one-body external potential f eld can represent the one-body fow induced force acting on the particles (see the end of section 3.4). Equation (44) implicitly assumes that a homogeneous one-body density distribution $n$ is not a function of the fow rate, thus neglecting possible dilation effects. Given equation (44), it is natural to go one step further and assume that the relation can be uniquely inverted, such that

$$
u(\mathbf{r}, \dot{\gamma})=u(\mathbf{r} ; g])
$$

By construction, $u(\mathbf{r}, \dot{\gamma})$ reproduces the nonequilibrium pair correlations. However, if the same fctitious two-body potential is used in an equilibrium statistical mechanical calculation of the triplet correlation function, the exact nonequilibrium $g^{(3)}$ will not be reproduced. The missing part of the triplet correlation is referred to as the 'irreducible' term

$g^{(3)}\left(\mathbf{r}_{1}, \mathbf{r}_{2}, \mathbf{r}_{3}, \dot{\gamma}\right)=g_{\text {eq }}^{(3)}\left(\mathbf{r}_{1}, \mathbf{r}_{2}, \mathbf{r}_{3} ;[g]\right)+g_{\mathrm{irr}}^{(3)}\left(\mathbf{r}_{1}, \mathbf{r}_{2}, \mathbf{r}_{3} ; \dot{\gamma}\right)$,

where we have assumed that (45) is valid. A key approximation in the work of [182] and [168] is to set $g_{\text {irr }}^{(3)}=0$, which essentially amounts to assuming that equilibrium relations such as (43) may be used to connect triplet and pair functions in nonequilibrium.

The nonequilibrium integral equation method considered in $[140,182,184]$ represents a synthesis of the exact dilute limit results with statistical mechanical descriptions of dense systems at equilibrium. While this approach is promising for weak fl ws $(P e \ll 1)$, data are lacking for stronger fows which would enable the close-to-equilibrium character of the closure approximations to be better tested. In particular, it has been emphasized by Szamel [168] that a major challenge for theories of the distorted microstructure is to account for 
the nonzero distortion of $g(\mathbf{r})$ in the vorticity-gradient plane perpendicular to an applied shear fow. This distortion cannot be detected to leading order in $P e$ and makes desirable numerical studies of the existing closure approximations under strong fow. Applications of the nonequilibrium integral equation method have been restricted to shear $\mathrm{fl} \mathrm{w}$ and, with the exception of small amplitude oscillatory shear [182, 184], have neglected time-dependent fows entirely.

6.3.5. Alternative approaches. The nonequilibrium pair correlations and rheology of colloidal dispersions under weak shear fl $\mathrm{w}$ were investigated by Szamel [168], who employed functional methods to approximate the unknown integral term in equation (25). Hydrodynamic interactions were neglected entirely. Assuming that the irreducible term in (46) can be neglected, the triplet correlation function can be developed in a functional Taylor expansion about the equilibrium state. To f rst order this is given by

$$
\begin{aligned}
& g^{(3)}\left(\mathbf{r}_{1}, \mathbf{r}_{2}, \mathbf{r}_{3} ;[g]\right)=g_{\text {eq }}^{(3)}\left(\mathbf{r}_{1}, \mathbf{r}_{2}, \mathbf{r}_{3}\right) \\
& +\int \mathrm{d} \mathbf{r}_{4} \int \mathrm{d} \mathbf{r}_{5} \delta g^{(3)}\left(\mathbf{r}_{1}, \mathbf{r}_{2}, \mathbf{r}_{3}\right)\left(g\left(\mathbf{r}_{4}, \mathbf{r}_{5}\right)-g_{\mathrm{eq}}\left(r_{45}\right)\right),
\end{aligned}
$$

where the functional derivative is evaluated for a constant onebody density. Equation (47) is clearly a close-to-equilibrium approximation. Insertion of (47) into the expression for the force (25) and rearrangement of terms leads to a closed equation for $g(\mathbf{r})$ which requires both $g_{\mathrm{eq}}^{(3)}\left(r_{12}, r_{23}, r_{13}\right)$ and $c_{\mathrm{eq}}^{(4)}\left(\mathbf{r}_{1}, \mathbf{r}_{2}, \mathbf{r}_{3}, \mathbf{r}_{4}\right)$, a higher order equilibrium direct correlation function, as input. Following appropriate decoupling approximations for the unknown higher order equilibrium correlations and linearizing with respect to the shear fow, Szamel obtained a closed equation for $g(\mathbf{r})$ which only requires $g_{\mathrm{eq}}(r)$ as input (for which the Verlet-Weiss approximation was employed [169]).

The Szamel theory [168] is considerably simpler to implement than the integral equation approach of Lionberger and Russel [182] and provides comparable, perhaps even slightly better, results for the zero-shear viscosity as a function of volume fraction, as is evident from fgure 12. Both $g(\mathbf{r})$ and its Fourier transform $S(\mathbf{k})$ were found to be qualitatively similar to those from the Lionberger-Russel theory, substantially underestimating the magnitude of the distortion from equilibrium when compared to Brownian dynamics simulation.

In an early study, Ronis took an alternative approach based on fuctuating hydrodynamics, in which phenomenological fuctuating terms are added to the macroscopic equations of hydrodynamics [187]. The theory of stochastic processes may then be employed to calculate nonequilibrium time correlation functions and the distorted microstructure. Of all the theories described in this and the previous section, the Ronis theory provides the most accurate results for the distortion of the structure factor at low $P e$ values. Nevertheless, it can be shown that the Ronis approximation leads to a vanishing of the microstructural distortion in the vorticity-gradient plane at all $P e$, in contradiction to experiment and simulation

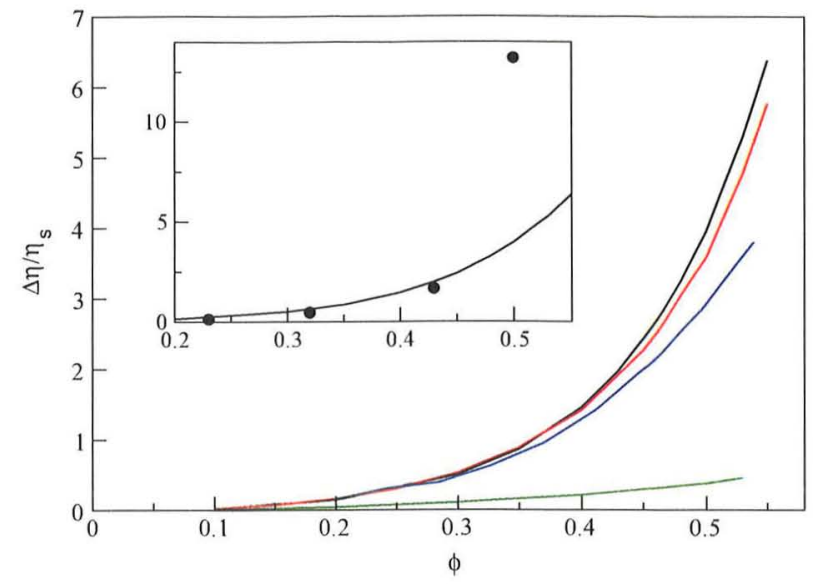

Figure 12. The reduced zero-shear viscosity as a function of volume fraction as predicted by various theories based on the pair Smoluchowski equation: Szamel (black) [168], Brady (red), Lionberger-Russel (blue) [182], Ronis (green) [187]. The inset shows the results of the Szamel theory compared with Brownian dynamics simulation data (circles). For $\phi>0.43$ the theory strongly underestimates the zero-shear viscosity. (Adapted from [168].)

results [159]. This def cit is related to the fact that the hardsphere 'core condition' $g(|\mathbf{r}|<1)=0$ is violated within this approach. The Ronis theory reduces to the closely related theory of Dhont [156] in the low Pe, linearized limit. In another early work, Schwarzl and Hess [188] postulated a phenomenological equation for $g(\mathbf{r})$ involving a number of empirical parameters representing the relaxation times in the system. However, due to the phenomenological nature of both the fuctuating hydrodynamics approach and the equationof-motion for $g(\mathbf{r})$ proposed by Schwarzl and Hess, the foundation of the theoretical treatments presented in [187] and [188] in nonequilibrium statistical mechanics is unclear.

Finally, we note that Wagner has assessed approaches based on the pair Smoluchowski equation using the GENERIC framework of beyond equilibrium thermodynamics [189]. This formalism enables any proposed closure of the pair Smoluchowski equation to be checked for thermodynamic consistency. The study presented in [189] identif ed the thermodynamically admissible expression for the stress tensor and clarif ed the nature of the inconsistencies which can occur when separate derivations are performed for the equation-ofmotion for $g(\mathbf{r})$ and the stress tensor.

\subsection{Temporal locality versus memory functions}

In sections 5.1 and 5.2 we introduced brief y the GreenKubo expressions for calculating the shear stress in both the linear (17) and nonlinear regimes (22). Within the GreenKubo framework, transport coeff cients are related to integrals over time correlation functions [191]. The growth of the shear viscosity as a function of volume fraction is thus related to an increasingly slow decay of the stress autocorrelation function. What is lacking thus far in the discussion of the pair Smoluchowski equation and its approximate solutions, is the connection between the temporally local 
equations (24) and (29) and the nonlocal expressions (17) and (22) fundamental to the time correlation function formalism. Moreover, the memory kernels characteristic of the time correlation approach (and widely employed in continuum mechanics approaches, see section 2) make no explicit appearance within the pair Smoluchowski framework.

The key to understanding the connection between the nonlocal time correlation formalism and approaches based on the local pair Smoluchowski equation lies in the study of time-dependent external fow felds. Indeed, it is the absence of time-dependent data from the pair Smoluchowski approach which has served to obscure the relationship between these two methods, despite the fact that they are formally equivalent. The only available Smoluchowski-based timedependent calculations were performed using the LionbergerRussel theory $[27,182,184]$ for small amplitude oscillatory shear fow. As pointed out in section 6.3 .4 , the available results for the volume fraction dependence of $G^{\prime}(\omega)$ and $G^{\prime \prime}(\omega)$ within the LR theory reveal underlying problems resulting from the approximations employed (i.e. a reduction of $\tau_{\alpha}$ with increasing $\phi$ ) which would otherwise have gone unnoticed. This serves to emphasize the importance of going beyond steady fow calculations in applications of pair Smoluchowski theories.

The generalization of equation (22) to general timedependent shear is given by integration over the entire fow history [16]

$$
\sigma_{x y}(t)=\int_{-\infty}^{t} \mathrm{~d} t^{\prime} \dot{\gamma}\left(t^{\prime}\right) G\left(t, t^{\prime}\right),
$$

where $G\left(t, t^{\prime}\right) \equiv G\left(t, t^{\prime} ;[\dot{\gamma}]\right)$ is the nonlinear shear modulus The lack of time-translational invariance in the modulus arises from a functional dependence on the shear rate. The microscopically derived equation (48) should be contrasted with the more familiar phenomenological result (2).

Using the generalized Green-Kubo result (48) it is instructive to consider a simple special case: the stress response of hard-spheres to the onset of steady shear fow in the absence of hydrodynamic interactions. Specif cally, we consider a shear f eld which is switched from zero to a constant value, $\dot{\gamma}(t)=\dot{\gamma} \Theta(-t)$. For this choice of shear feld equation $(\$ 8)$ reduces to

$$
\sigma_{x y}(t)=\dot{\gamma} \int_{0}^{t} \mathrm{~d} t^{\prime} G_{\mathrm{ss}}\left(t^{\prime}\right),
$$

where $G_{s \mathrm{~s}}(t)$ is the time translationally invariant shear modulus under steady shear fow. On the other hand, within approaches based on the distorted microstructure the interaction contribution to the stress is given by (see equation (29))

$$
\sigma_{x y}(t)=-\frac{n^{2}}{2} \int \mathrm{d} \mathbf{r} \underset{r}{\mathbf{r r}} v^{\prime}(r) g(\mathbf{r}, t)
$$

where $g(\mathbf{r}, t)$ is calculated from the pair Smoluchowski equation (24) subject to the switch-on shear fow under consideration. The expressions (49) and (50) are formally equivalent. Equating the time derivatives thus leads to the exact relation

$$
G_{\mathrm{ss}}(t)=-{ }_{2 \dot{\gamma}}^{n^{2}} \int \mathrm{d} \mathbf{r} \underset{r}{\mathbf{r r}} v^{\prime}(r) \underset{\partial t}{\partial g(\mathbf{r}, t)} .
$$

The quiescent shear modulus is thus recovered in the slow fow limit

$$
G_{\mathrm{eq}}(t)=-{ }_{2}^{n^{2}} \int \mathrm{d} \mathbf{r}{ }_{r}^{\mathbf{r r}} v^{\prime}(r) \lim _{\dot{\gamma} \rightarrow 0}\left(\begin{array}{cc}
1 & \partial g(\mathbf{r}, t) \\
\dot{\gamma} & \partial t
\end{array}\right) .
$$

The right-hand side of equation (52) can be further reduced to the determination of the function $f(r, t)$ by substitution of the first order expansion (31) for $g(\mathbf{r}, t)$.

As the volume fraction is increased, the time derivative on the rhs of equation (52) must give rise to a growth in the timescale determining the relaxation of stress fuctuations, as described by $G_{e q}(t)$. The fact that this is not captured by the Lionberger-Russel theory $[27,182,184]$ simply reflec $s$ the failings of the approximate closure (43) relating the nonequilibrium triplet and pair correlation functions. However, it is not clear that even an exact equilibrium expression for the triplet correlations would be suff cient to resolve these diff culties. If the key source of error lies in the neglect of the irreducible term appearing in (46) then considerable new insight into the nature of nonequilibrium states will be required in order to make further progress using integral equation methods. Nevertheless, these considerations should serve to motivate time-dependent studies of the Russel-Gast [1 41$]$ and Szamel $[168]$ theories.

While the particular example chosen (switch-on shear fl w) provides access to the steady shear rate dependent modulus, other choices of time-dependence may enable connection to be made between non-time translationally invariant correlation functions (e.g. $G\left(t, t^{\prime}\right)$ ) and time-dependent solutions of the pair Smoluchowski equation. The issue of whether temporally local constitutive equations are preferable to nonlocal functionals for describing complex fuids has been addressed within the framework of nonequilibrium thermodynamics [190]. The nonequilibrium thermodynamics approach requires identif cation of an appropriate set of structural variables which, in addition to the standard hydrodynamic variables of mass, momentum and internal energy density, contain all information about the state of the system at a given time that is necessary to determine the macroscopic quantities of interest. The pair Smoluchowski approaches discussed in this section essentially introduce $g(\mathbf{r})$ as an additional structural variable [189]. While this appears to be a valid approach for low and intermediate volume fractions, a correct identif cation of the structural variables appropriate for describing glass formation and dynamical arrest remains to be found. For this reason the most promising approaches to treating high volume fraction states are based on the generalized Green-Kubo relations and mode-coupling theory.

\section{Glass rheology}

Assuming that crystallization effects can be suppressed (see section 4) the volume fraction can be increased to the 


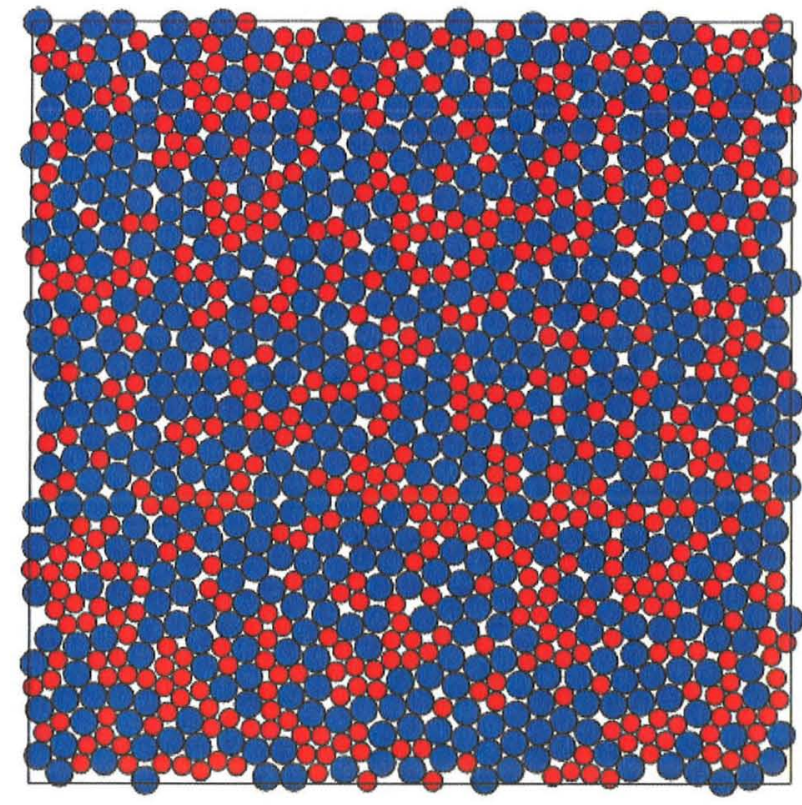

Figure 13. A snapshot from a Brownian dynamics simulation of a quiescent binary hard-disc mixture (using a size ratio of $1: 1.4$ to supress crystallization). The simulation was performed at a two-dimensional volume fraction of $\phi_{2 \mathrm{D}}=0.81$, which lies above the estimated glass transition packing $\phi_{2 D}^{(\mathrm{g})} \sim 0.79$, with $50 \%$ large discs and $50 \%$ small discs. (Figure courtesy of F Weyßer.)

point at which the individual particles are unable to diffuse beyond the cage of nearest neighbours and a dynamically arrested glassy state is formed. In order to visualize the amorphous cage structure in such a glassy state f gure 13 shows a conf guration snapshot taken from a Brownian dynamics simulation of a binary hard-sphere mixture in two dimensions (hard-discs) [75]. The two-dimensional volume fraction $\phi_{2 \mathrm{D}}=$ 0.81 of the simulation is above the estimated glass transition point of $\phi_{2 \mathrm{D}} \approx 0.79$ and the size ratio of large to small disc radii is 1.4 , a value empirically found to frustrate crystallization in two-dimensional systems (which occurs at $\phi_{2 \mathrm{D}}=0.69$ for monodisperse discs). In both two- and three-dimensional systems the physics of the glass transition becomes important for determination of both the rheology and fow distorted microstructure of high volume fraction systems.

The response to externally applied fow of states close to, or beyond, the glass transition is only beginning to be understood and establishing the basic principles of glass rheology remains a challenging task. At present, the only truly microscopic theories available are provided by recent extensions of the quiescent MCT which enable the effects of external fow to be incorporated into the formalism and thus make possible a theoretical investigation of the complex interaction between arrest and fow.

\subsection{MCT inspired approaches}

Extending earlier work on the low volume fraction selfdiffusion of colloidal dispersions [192], Miyazaki and Reichman constructed a self-consistent mode-coupling-type approach to describe collective density fl ctuations for dense colloidal fuids under shear below the glass transition [193-195]. The Miyazaki-Reichman theory considers time-dependent fuctuations about the steady state and thus requires the (unknown) fow distorted structure factor $S(\mathbf{k})$ as an input quantity. Approximating $S(\mathbf{k})$ by the quiescent correlator, results have been presented for colloidal dispersions in two dimensions under steady shear $[193,194]$ and in three dimensions (subject to additional isotropic approximations) under oscillatory shear [195]. Applications to glassy states have been avoided as the theory relies upon an ergodic f uctuation-dissipation theorem. An alternative extended-MCT approach has been proposed by Koblev and Schweizer [196] and Saltzman et al [197] which is built on the idea that entropic barrier hopping is the key physical process driving the microscopic dynamics and rheology of glassy colloidal suspensions. Due to the activated nature of the barrier hopping process the ideal glass transition described by quiescent MCT (see section 4) plays no role. A nonlinear rheological response results from a stress induced modifcation of the barrier heights.

A currently promising method of extending quiescent MCT to treat dense systems under fow involves integration through the transient dynamics, starting from an equilibrium Boltzmann distribution in the inf nite past. In contrast to [193-195] the distorted microstructure is an output of this approach. The initial form of the theory was outlined by Fuchs and Cates for steady shear fl w [135] and presented two essential developments: frstly, that integration through the transient dynamics leads directly to exact generalized Green-Kubo formulae, relating average quantities to integrals over microscopic time correlation functions. Secondly, that MCT-type projection operator approximations reduce the formal Green-Kubo expressions to closed equations involving transient correlators (which can be calculated self-consistently). A strong prediction of the ITT-MCT theory resulting from combining these two steps is that the macroscopic fow curves exhibit a dynamic yield stress (see section 5.4) in the limit $P e \rightarrow 0$, for states which would be glasses or gels in the absence of fow. Moreover, the yield stress appears discontinuously as a function of volume fraction, in contrast to mesoscopic approaches [34-36]. The ITTMCT thus provides a scenario for a nonequilibrium transition between a shear thinning fuid and a yielding amorphous solid which is supported by considerable evidence from both colloidal experiments $[60,61,134,198]$ and Brownian dynamics simulation $[40,75]$.

Due to the numerical intractability of the microscopic theory of [135], subsequent work focused on the construction of both isotropically averaged approximations to the full anisotropic equations and simplif ed schematic models inspired by these [57]. Comparison of the theoretical predictions with experimental data for thermosensitive core-shell particles (see f gure 7) has proved highly successful [60, 61, 199-201]. The original formulation of the ITT-MCT (more details of which can be found in [202]) has subsequently been superseded by a more elegant version [20]. It is interesting to note that the signif cant technical changes to the ITTMCT formalism introduced in [20] lead to expressions 
which resemble more closely those of Miyazaki and Reichman [193-195]. Given the very different nature of the formal derivation (f uctuating hydrodynamics versus projection operator methods) and approximations employed, the similarity of the f nal expressions is reassuring and serves to highlight the robustness of MCT-based approaches. For a comprehensive overview of the status of the steady shear theory we refer the reader to the recent review [19].

Going beyond steady shear, the original formulation of ITT-MCT $[57,135,202]$ has been generalized to treat arbitrary time-dependent shear [16]. These developments not only enable shear fie ds of particular experimental relevance to be investigated (e.g. large amplitude oscillatory shear fow), but have also revealed an underlying mathematical structure which is not apparent from consideration of steady fows alone. The theory has been applied (albeit subject to various simplifying approximations) to investigate the build-up of stress and corresponding microscopic particle motion, as encoded in the mean-squared-displacement, following the onset of shear [62]. More recently, the modern version of ITT-MCT [20] has been extended to describe time-dependent fow of arbitrary geometry [17], thus making possible the study of non-shear fow and enabling the full tensorial structure of the theory to be identif ed. The developments presented in [17] elevate the ITT-MCT approach to the level of a full constitutive theory for dispersion rheology and may be regarded as the most up-to-date formulation of the theory. While the development of numerical algorithms to eff ciently solve the fully microscopic theory [17] is currently in progress, this task is made computationally dentanding by the combination of spatial anisotropy and lack of time-translational invariance presented by many fows of interest. In [18] a simplif ed theory was presented which contains the essential physics of the full microscopic equations, including the tensorial structure, but which is much more convenient for numerical solution (see section 7.7).

\subsection{Integration through transients}

The integration through transients (ITT) approach originally developed by Fuchs and Cates [135] and subsequently developed in $[16,17]$ provides a formal expression for the nonequilibrium distribution function $\Psi(t)$ required to calculate average quantities under fow. In essence, ITT provides a very elegant method of deriving generalized (i.e. nonlinear in $\kappa(t))$ Green-Kubo relations which invite mode-coupling-type closure approximations. The current formulation of the theory neglects hydrodynamic interactions (HI) entirely. On one hand this omission is made for purely technical reasons, but it is also hoped that HI will prove unimportant for the microscopic dynamics of the dense states to which the f nal theory will be applied. In the following we will briefy outline the key points of ITT, employing throughout the modern formulation of [17].

The starting point for ITT is to re-express the Smoluchowski equation (14) in the form

$$
\begin{gathered}
\partial \Psi(t) \\
\partial t
\end{gathered}=\Omega(t) \Psi(t)
$$

where, in the absence of $\mathrm{HI}$, the Smoluchowski operator controlling the dynamical evolution of the system is given by $[14]$

$$
\Omega(t)=\sum_{i} \partial_{i} \cdot\left[D_{0}\left(\partial_{i}-\beta \mathbf{F}_{i}\right)-\kappa(t) \cdot \mathbf{r}_{i}\right] .
$$

Equation (53) may be formally solved using a time-ordered exponential function (which arises because $\Omega(t)$ does not commute with itself for different times [203])

$$
\Psi(t)=\left[\exp _{+} \int_{-\infty}^{t} \mathrm{~d} s \Omega(s)\right] \Psi_{\mathrm{eq}},
$$

where $\Psi_{\mathrm{eq}}$ is the initial distribution function in the inf nite past, which is taken to be the equilibrium Boltzmann-Gibbs distribution corresponding to the thermodynamic state point under consideration. The assumption of an equilibrium distribution is clearly acceptable in situations for which the quiescent state is one of ergodic equilibrium. However, the role of the initial state is less clear for statepoints in the glass and the dependence, if any, on the initial condition may depend upon the details of the fow between $t=-\infty$ and the present time $t$. The absence of a general proof that $\Psi(t)$ is independent of $\Psi(-\infty)$ leaves open the possibility that certain fow histories do not restore ergodicity and that the system thus retains a dependence on the initial state.

The solution (55) is formally correct, but not particularly useful in its present form. A partial integration yields an alternative solution of (53) which is exactly equivalent to (55), but more suited to approximation

$$
\Psi(t)=\Psi_{e}+\int_{-\infty}^{t} \mathrm{~d} t_{1} \Psi_{e} \kappa\left(t_{1}\right): \hat{\sigma} \mathrm{e}_{-}^{l_{f_{1}}^{t_{1}} \mathrm{~d} s \Omega^{\dagger}(s)},
$$

where $\hat{\sigma}_{\alpha \beta}=-\sum_{i} F_{i}^{\alpha} r_{i}^{\beta}$ and the "double $\operatorname{dot}^{\text {' notation }}$ familiar from continuum mechanics, $\mathbf{A}: \mathbf{B}=\operatorname{Tr}\{\mathbf{A} \cdot \mathbf{B}\}$ [204], has been employed. As a result of the partial integration the dynamical evolution in equation (56) is dictated by the adjoint Smoluchowski operator

$$
\Omega^{\dagger}(t)=\sum_{i}\left[D_{0}\left(\partial_{i}+\beta \mathbf{F}_{i}\right)+\mathbf{r}_{i} \cdot \kappa^{\mathrm{T}}(t)\right] \cdot \partial_{i} .
$$

Equation (56) is the fundamental formula of the ITT approach and expresses the nonequilibrium distribution function as an integral over the entire transient fow history. Both solutions (55) and (56) are valid for arbitrary fow geometries and time-dependence. The relation between the two formal solutions is analogous to the Heisenberg and Schrödinger pictures of quantum mechanics in which the time evolution of the system is attributed to either the wavefunction (equation (55)) or the operators representing physical observables (equation (56)). It should be understood that the ITT form (56) is an operator expression to be used with the understanding that a function to be averaged is placed on the right of the operators and integrated over the particle coordinates. A technical point to note is that in cases for which phase space decomposes into disjoint pockets ('nonmixing' dynamics) the distribution (56) averages over 
all compartments. A general function of the phase space coordinates $f\left(t,\left\{\mathbf{r}_{i}\right\}\right)$ thus has the average

$$
\langle f\rangle^{\mathrm{ne}}=\langle f\rangle+\int_{-\infty}^{t} \mathrm{~d} t_{1}\left\langle\kappa\left(t_{1}\right): \hat{\sigma} \mathrm{e}_{-}^{f_{t_{1}}^{t} \mathrm{~d} s \Omega^{\dagger}(s)} f\right\rangle,
$$

where $\langle f\rangle^{\text {ne }}$ denotes an average over the nonequilibrium distribution (56). Equation (56) generalizes the original formulation of ITT [135] to treat arbitrary time-dependence [17].

\subsection{Translational invariance}

Before applying mode-coupling-type approximations to the exact result (58) we frst address an important consequence of assuming homogeneous fow (ref ected in the spatial constancy $\kappa(t)$ appearing in equation (53)). On purely physical grounds, it seems reasonable that for an inf nite system the assumed translational invariance of the equilibrium state (crystallization is neglected) will be preserved by the Smoluchowski dynamics. However, proving this for a general time-dependent fow is mathematically nontrivial, due to the fact that the Smoluchowski operator (54) is itself not translationally invariant. By considering a constant vectorial shift of all particle coordinates, $\mathbf{r}_{i}^{\prime}=\mathbf{r}_{i}+\mathbf{a}$, Brader et al have shown that the nonequilibrium distribution function $\Psi(t)$ is translationally invariant (but anisotropic) for any homogeneous velocity gradient $\kappa(t)$ [17]

Given the translational invariance of $\Psi(t)$ it becomes possible to investigate the more interesting invariance properties of the two-time correlation functions. The correlation between two arbitrary wavevector-dependent fuctuations $\delta f_{q}=f_{q}-\left\langle f_{q}\right\rangle^{\text {ne }}$ and $\delta g_{k}=g_{k}-\left\langle g_{k}\right\rangle^{\text {ne }}$ occurring at times $t$ and $t^{\prime}$ is given by

$$
C_{\mathrm{f}_{\mathrm{q}} g_{\mathbf{k}}}\left(t, t^{\prime}\right)=\left\langle\delta f_{\mathbf{q}}^{*}(t) \delta g_{\mathbf{k}}\left(t^{\prime}\right)\right\rangle^{\text {ne }} .
$$

It is clear that in a homogeneous system the correlation function (59) must be translationally invariant. However, in this case, shifting the particle coordinates by a constant vector a yields

$$
C_{\mathrm{f}_{\mathrm{q}} g_{\mathrm{k}}}\left(t, t^{\prime}\right)=\mathrm{e}^{-\mathrm{i}\left(\overline{\mathrm{q}}\left(t, t^{\prime}\right)-\mathrm{k}\right) \cdot \mathrm{a}} C_{\mathrm{f}_{\mathrm{q}} g_{\mathrm{k}}}\left(t, t^{\prime}\right),
$$

where

$$
\overline{\mathbf{q}}\left(t, t^{\prime}\right)=\mathbf{q} \cdot \mathrm{e}_{-}^{-\int_{t^{\prime}}^{t} \mathrm{~d} s \kappa(s)} .
$$

The only way in which the required translational invariance of the correlation function can be preserved is if the exponential factor in (60) is equal to unity. This requirement has the consequence that a $\mathrm{f}$ uctuation at wavevector $\mathbf{k}=\overline{\mathbf{q}}\left(t, t^{\prime}\right)$ at time $t^{\prime}$ is correlated with a fuctuation with wavevector $\mathbf{q}$ at time $t$ as a result of the aff ne solvent fow. Equation (61) thus def nes the advected wavevector which is central to the ITT-MCT approach and which captures the affi e evolution of the system in approaches focused on Fourier components of f uctuating quantities (e.g. the density $\rho_{\mathrm{k}}$ ) rather than particle coordinates directly. The wavevector $\overline{\mathbf{q}}\left(t, t^{\prime}\right)$ at time $t^{\prime}$ evolves due to $\mathrm{fl} w$ induced advection to become $q$ at later time $t$. It should be noted that various def nitions and notations for the advected wavevector have been employed in the literature documenting the development of ITT-MCT and which could provide a source of confusion. In the present work we exclusively employ the modern def nition used in $[17,18,20]$.

Although equation (61) arises from microscopic considerations it is nevertheless fully consistent with the continuum mechanics approaches outlined in section 2 , despite the very different mindset underlying the two methods. Equation (61) simply describes the aff ne deformation of material lines in Fourier space and can thus be used to def ne the inverse deformation gradient tensor via $\overline{\mathbf{q}}\left(t, t^{\prime}\right)=\mathbf{q} \cdot \mathbf{E}^{-1}\left(t, t^{\prime}\right)$ in complete accord with continuum approaches. Doing so leads to the identif cation

$$
\mathbf{E}^{-1}\left(t, t^{\prime}\right)=\mathrm{e}_{-}^{-\int_{t^{\prime}}^{t} \mathrm{~d} s k(s)}
$$

As the deformation gradient tensor simply describes the aff ne distortion of a material line under fow, it is natural to def ne also a reverse-advected wavevector resulting from the inverse transformation $\mathbf{q}\left(t, t^{\prime}\right)=\mathbf{q} \cdot \mathbf{E}\left(t, t^{\prime}\right)$, where

$$
\mathbf{E}\left(t, t^{\prime}\right)=\mathrm{e}_{+}^{\int_{t^{\prime}}^{t} \mathrm{~d} s \kappa(s)} .
$$

The choice of using either advected or reverse-advected wavevectors in treating the effects of aff ne motion within a microscopic theory has parallels with the choice between Lagrangian and Eulerian specifcations of the fow feld in continuum fuid dynamics approaches [30]. Within a continuum mechanics framework the deformation gradient would simply be defined as the solution of the equation

$$
{ }_{\partial t}^{\partial} E\left(t, t^{\prime}\right)=\kappa(t) E\left(t, t^{\prime}\right),
$$

for a given fow $\kappa(t)$. According to the rules of timeordered exponential algebra [203], equation (63) is the formal solution of (64), thus demonstrating the consistency between the Fourier-space microscopic approach of [17] and traditional real-space continuum mechanics.

The advected wavevector introduced above provides a convenient way to keep track of the aff ne deformation in a particulate system. Mode-coupling-type approximations (to be discussed below) seek to factorize the average entering equation (58) by projecting the dynamics onto the subspace of density fuctuations $\rho_{\mathrm{q}}$ [98]. For a fowing system a f uctuation at wavevector $\bar{q}\left(t, t^{\prime}\right)$ at time $t^{\prime}$ evolves (in the absence of interactions and Brownian motion) to one at $\mathbf{q}$ at time $t$. It thus becomes essential to project onto density fuctuations at the correct advected wavevectors in order to avoid spurious decorrelation effects in the resulting approximations.

\subsection{Microscopic constitutive equation}

In order to address dispersion rheology the special choice $f=$ $\hat{\sigma} / V$ is made in (58), leading to an exact generalized GreenKubo relation for the time-dependent shear stress tensor [17]

$$
\sigma(t)=\frac{1}{V} \int_{-\infty}^{t} \mathrm{~d} t_{1}\left\langle\kappa\left(t_{1}\right): \hat{\sigma} \mathrm{e}_{-}^{\int_{l_{1}}^{t} \mathrm{~d} s \Omega^{\dagger}(s)} \hat{\boldsymbol{\sigma}}\right\rangle,
$$

noting that there are no 'frozen in' stresses in the equilibrium state, $\langle\sigma\rangle=0$. The adjoint Smoluchowski operator $\Omega^{\dagger}(t)$ has 
a linear dependence on $\kappa(t)$ and equation $(65)$ is thus nonlinear in the velocity gradient tensor. Equation (65) is a formal constitutive equation expressing the stress tensor at the present time as a nonlinear functional of the fow history. Although the result (65) does not provide an exact description of the physical system under consideration (particle momenta are assumed to have relaxed and hydrodynamic interactions are absent), it has a formal status equivalent to that of equation (56). For the special case of steady shear fl $\mathrm{w}(65)$ is consistent with (22) with a shear modulus given by $(23)$.

Application of MCT-type projection operator factorizations [17] to the average in (65) leads to a complicated, but closed, constitutive equation expressing the deviatoric stress in terms of the strain history $[17,18]$

$$
\begin{aligned}
\sigma(t) & =-\int_{-\infty}^{t} \mathrm{~d} t^{\prime} \int_{32 \pi^{3}} \mathrm{~d} \mathbf{k}\left[\begin{array}{c}
\partial \\
\partial t^{\prime}
\end{array}\left(\mathbf{k} \cdot \boldsymbol{B}\left(t, t^{\prime}\right) \cdot \mathbf{k}\right) \mathbf{k k}\right] \\
& \times\left[\left(\begin{array}{c}
S_{k}^{\prime} S_{k\left(t, t^{\prime}\right)}^{\prime} \\
k k\left(t, t^{\prime}\right) S_{k}^{2}
\end{array}\right) \Phi_{\mathbf{k}\left(t, t^{\prime}\right)}^{2}\left(t, t^{\prime}\right)\right]
\end{aligned}
$$

where $S_{k}$ and $S_{k}^{\prime}$ are the equilibrium static structure factor and its derivative, respectively. The inf uence of external fow enters both explicitly, via the Finger tensor $\mathbf{B}\left(t, t^{\prime}\right)$ (see section 2.1), and implicitly through the reverse-advected wavevector. As noted above, the reverse-advected wavevector, which provides an important source of nonlinearity in (66), enters as a result of judicious projection of the dynamics onto appropriately advected density fuctuations $\rho_{\mathrm{k}\left(\mathbf{t}, \mathbf{t}^{t^{\prime}}\right)}$. The normalized transient density correlator describes the decay under fow of thermal density fuctuations and is def ned by

$$
\Phi_{\mathrm{k}}\left(t, t^{\prime}\right)=\begin{gathered}
1 \\
N S_{k}
\end{gathered}\left\langle\rho_{\mathrm{k}}^{*} \mathrm{e}_{-}^{\int_{t^{\prime}}^{t} \mathrm{~d} s \Omega^{\dagger}(s)} \rho_{\overline{\mathbf{k}\left(t, t^{\prime}\right)}}\right\rangle
$$

The occurrence of the advected wavevector in (67) ensures that trivial decorrelation effects are removed (i.e. that in the absence of Brownian motion and potential interactions $\Phi_{\mathrm{k}}=1$ for all times).

In order to close the constitutive equation (66) we require an explicit expression for the transient correlator $(67)$. Time-dependent projection operator manipulations combined with the theory of Volterra integral equations yield an exact equation-of-motion for the time evolution of the transient correlator containing a generalized friction kernel-a memory function formed from the autocorrelation of fuctuating stresses. Mode-coupling-type approximations to this kernel yield the nonlinear integro-differential equation [16-18]

$$
\begin{aligned}
& \dot{\Phi}_{\mathbf{q}}\left(t, t_{0}\right)+\Gamma_{\mathbf{q}}\left(t, t_{0}\right)\left(\Phi_{\mathbf{q}}\left(t, t_{0}\right)\right. \\
& \left.\quad+\int_{t_{0}}^{t} \mathrm{~d} t^{\prime} m_{\mathbf{q}}\left(t, t^{\prime}, t_{0}\right) \dot{\Phi}_{\mathbf{q}}\left(t^{\prime}, t_{0}\right)\right)=0
\end{aligned}
$$

where the overdots denote partial differentiation with respect to the frst time argument. Here the 'initial decay rate' obeys $\Gamma_{\mathrm{q}}\left(t, t_{0}\right)=D_{0} \bar{q}^{2}\left(t, t_{0}\right) / S_{\bar{q}\left(t, t_{0}\right)}$ with $D_{0}$ the diffusion constant of an isolated particle. The formal manipulations presented in $[16,17]$ have revealed that imposing a time-dependent fow results in a memory kernel which depends upon three time arguments. The presence of a third time argument, which would have been diff cult to guess on the basis of quiescent MCT intuition, turns out to have important consequences for certain rapidly varying fows (e.g. step strain [16]) and is essential to obtain physically sensible results in such cases. The memory kernel $m_{\mathrm{q}}\left(t, t^{\prime}, t_{0}\right)$ entering $(68)$ is given by the factorized expression $[17,18]$

$$
\begin{aligned}
& m_{\mathbf{q}}\left(t, t^{\prime}, t_{0}\right)=\underset{16 \pi^{3}}{\rho} \int \mathrm{d} \mathbf{k} \begin{array}{c}
S_{\tilde{q}\left(t, t_{0}\right)} S_{\tilde{k}\left(t^{\prime}, t_{0}\right)} S_{\bar{p}\left(t^{\prime}, t_{0}\right)} \\
\bar{q}^{2}\left(t^{\prime}, t_{0}\right) \bar{q}^{2}\left(t, t_{0}\right)
\end{array} \\
& \times V_{\mathrm{qkp}}\left(t^{\prime}, t_{0}\right) V_{\mathrm{qkp}}\left(t, t_{0}\right) \Phi_{\overline{\mathrm{k}}\left(t^{\prime}, t_{u}\right)}\left(t, t^{\prime}\right) \Phi_{\tilde{\mathrm{p}}\left(t^{\prime}, t_{0}\right)}\left(t, t^{\prime}\right),
\end{aligned}
$$

where $\mathbf{p}=\mathbf{q}-\mathbf{k}$, and the vertex function obeys

$$
V_{\mathrm{qkp}}\left(t, t_{0}\right)=\overline{\mathbf{q}}\left(t, t_{0}\right) \cdot\left(\overline{\mathbf{k}}\left(t, t_{0}\right) c_{\bar{k}\left(t, t_{0}\right)}+\overline{\mathbf{p}}\left(t, t_{0}\right) c_{\bar{p}\left(t, t_{0}\right)}\right)
$$

with Ornstein-Zernike direct correlation function $c_{k}=1-$ $1 / S_{k}$ (see equation (39)). In the linear regime equations (66) and $(68)$ reduce to the standard quiescent MCT forms (19) and $(2 \mathrm{l})$, respectively.

An important feature of equations $(66)-(70)$ is that they offer a closed constitutive equation requiring only the static structure factor and velocity gradient tensor $\kappa(t)$ as input to calculating the stress tensor. The equilibrium $S_{\mathrm{q}}$ is determined by the interaction potential and thermodynamic statepoint and, as in quiescent MCT, serves as proxy for the pair potential (an interpretation arising from feld-theoretical approaches to MCT [205]). The role of $S_{9}$ within the ITT-MCT should be contrasted with that within the Miyazaki-Reichman theory [193-195], discussed in section 7.1, where it enters as an approximation to the fow distorted structure factor $S(\mathbf{k})$.

In section 2.3 we introduced the principle of material objectivity; an approximate symmetry requiring that a valid constitutive relation be rotationally invariant. While verif cation of rotational invariance is straightforward for the phenomenological Lodge equation introduced in section 2.1, proof becomes more demanding for the microscopic constitutive theory given by equations (66)-(70). Nevertheless, substitution of equations (11) and (13) into (66)-(70) yields the result (12), thus verifying that the ITT-MCT constitutive equation is indeed material objective as desired [17]. Material objectivity is an important consistency check for constitutive theories based on overdamped Smoluchowski dynamics, for which it represents an exact symmetry constraint.

Possibly the most exciting feature of the ITT-MCT constitutive equation $(66)-(70)$ is that it incorporates a mechanism for describing the slow structural relaxation leading to dynamical arrest. The predicted rheological response thus goes from that of a viscous fuid to that of an amorphous solid, characterized by an elastic constant, upon variation of the thermodynamic control parameters. The ability of the theory to unify the description of fuid and glassy states stems from the underlying mode-coupling-type approximations which are tailored to capture the cooperative particle motion in dense colloidal dispersions, ultimately leading to particle caging and arrest. Mathematically, this scenario arises from a bifurcation in the solution of the nonlinear integro-differential equation (68) at suff ciently high volume fraction/attraction strength associated with a diverging relaxation time of the transient density correlator. Glass formation within the MCT-ITT approach is a purely 
dynamical phenomenon, as the equilibrium $S_{q}$ used as input varies smoothly across the transition. The fuid-solid transition contained within the ITT-MCT equations can be better appreciated by considering the small strain limit of equation (66) which yields the linear response result [17]

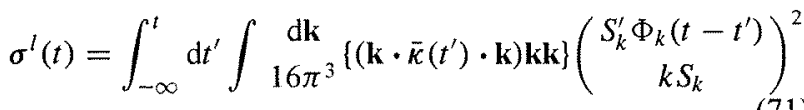

where $\Phi_{k}(t)$ is the correlator from quiescent MCT [98]. In the glass the correlator does not relax to zero for long times and a partial integration of (71) followed by taking the limit of small strain leads to the result

$$
\sigma(t)=2 G(t \rightarrow \infty) \epsilon(t),
$$

where $\epsilon(t)$ is the inf nitesimal strain tensor and $G(t \rightarrow \infty)$ is an elastic modulus obtained from equation (19) (also known as Lamé's second coeffic ent, the fir $t$ being zero here due to incompressibility). Equation (72) is essentially Hooke's law, describing the small strain response of a glassy solid. Going beyond linear response, equation (66) incorporates the f uidizing effect of fow and thus makes possible investigation of a large number of time-dependent rheological situations in which externally applied fow felds compete with glass formation and slow structural relaxation (see section 7.6).

\subsection{Distorted structure factor}

The microscopic ITT-MCT constitutive equation discussed above enables comparisons to be made with traditional continuum rheological modelling (section 2) for which the macroscopic stress tensor is the fundamental quantity of interest. However, the formal ITT result (58) also enables calculation of the distorted structure factor, $S(\mathbf{k}, t)=1+$ $n \int \mathrm{d} \mathbf{r}(g(\mathbf{r}, t)-1) \mathrm{e}^{\mathrm{i} \cdot \mathbf{r}}$, which makes possible a comparison with the microstructure obtained from approaches based on the pair Smoluchowski equation (section 6.1). In particular, setting $f=\Delta \rho_{\mathbf{k}}^{*} \rho_{\mathbf{k}} \equiv \rho_{\mathbf{k}}^{*} \rho_{\mathbf{k}}-\left\langle\rho_{\mathbf{k}}^{*} \rho_{\mathbf{k}}\right\rangle$ in equation (58) yields the formal result

$$
S(\mathbf{k}, t)=\left\langle\rho_{\mathbf{k}}^{*} \rho_{\mathbf{k}}\right\rangle+\int_{-\infty}^{t} \mathrm{~d} t^{\prime}\left\langle\kappa\left(t^{\prime}\right): \hat{\sigma} \mathrm{e}_{-}^{\int_{t^{\prime}}^{t} \mathrm{~d} s \Omega^{\dagger}(s)} \Delta \rho_{\mathbf{k}}^{*} \rho_{\mathbf{k}}\right\rangle
$$

Mode-coupling projection operator steps analogous to those leading to (66) yield the ITT-MCT expression for the distorted structure factor

$$
S(\mathbf{k}, t)=S_{k}-\int_{-\infty}^{t} \mathrm{~d} t^{\prime} \underset{\partial t^{\prime}}{\partial S_{k\left(t, t^{\prime}\right)}} \Phi_{\mathbf{k}\left(t, t^{\prime}\right)}^{2}\left(t, t^{\prime}\right)
$$

where the transient density correlator is given by solution of equations $(68)-(70)$ for given $S_{k}$ and $\kappa(t)$ and where an isotropic term has been suppressed. Equation (74) has the appealing interpretation that fow induced microstructural changes are built-up by integration of the aff nely shifted equilibrium structure factor over the entire fow history, weighted by the transient density correlator describing the fading memory of the system. The temporally nonlocal character of equation (74) is in striking contrast to the local approximations based on the pair Smoluchowski equation. The former consists of a history integral over a memory function which is itself determined by solution of a nonlocal integro-differential equation (68) whereas the latter are purely Markovian approximations.

Within the pair Smoluchowski approach, in the absence of HI the stress tensor is exactly related to the distorted pair correlation function by equation (29). It is therefore of interest to enquire whether a similar connection holds within the approximate ITT-MCT approach. It is a relatively simple exercise to show that, subject to a certain constraint to be discussed below, equations (66) and (74) are connected by the relation $[17]$

$$
\sigma(t)=-\Pi 1-n k_{\mathrm{B}} T \int \underset{16 \pi^{3} \quad k}{\mathrm{~d} \mathbf{k}} \mathbf{k} \mathbf{k} c_{k}^{\prime} \delta S_{\mathrm{k}}(t)
$$

where $\delta S_{\mathrm{k}}(t ; \kappa)=S_{\mathrm{k}}(t ; \kappa)-S_{k}$ and $\Pi$ is the equilibrium osmotic pressure. For shear fow $\sigma_{x y}(t)$ from (75) coincides with a result of Fredrickson and Larson [206] for sheared copolymers, refecting the Gaussian statistics underlying both the feld-theory approach of [206] and the ITT-MCT factorization approximations. Equation (75) thus connects stresses to microstructural distortions, which build up over time via the aff ne stretching of density fuctuations competing with structural rearrangements encoded in $\Phi_{\mathrm{k}}\left(t, t^{\prime}\right)$.

For the off-diagonal stress tensor elements equation (75) connects equations (66) and (74) directly. For the diagonal elements contributing to the osmotic pressure equation (75) is also valid, providing that the following approximate "sum-rule" is obeyed

$$
\frac{\partial \Pi}{\partial \phi}=\begin{gathered}
1 \\
6 \pi
\end{gathered} \int \mathrm{d} \mathbf{k}\left(\begin{array}{c}
\partial \ln S_{k} \\
\partial \ln k
\end{array}\right)\left(\begin{array}{c}
\partial \ln S_{k} \\
\partial \ln \phi
\end{array}\right) .
$$

Although it is not at all obvious that the above relation should hold, numerical calculations for hard-spheres (using e.g. the Percus-Yevick approximation for $S_{k}$ [15]) show that it represents a rather good approximation. It should be emphasized that the application of projection operator approximations to (65) and (73) yields approximate expressions for the stress and structure factor, respectively, which are not necessarily self-consistent, in the sense that integration of the approximate $S(\mathbf{k}, t)$ leads to the approximate $\sigma(t)$. The fact that the ITT-MCT $S(\mathbf{k}, t)$ is almost consistent with the direct ITT-MCT approximation to the stress is a testament to the underlying robustness of the method.

Although the caging mechanism is expected to be most important for statepoints close to the glass transition, ITTMCT calculations of $S(\mathbf{k})$ for hard-spheres under weak shear fl $\mathrm{W}$ [185] suggest that the (truncated) divergence of the structural relaxation timescale at the point of dynamical arrest (be it at the idealized glass transition point, as predicted by $\mathrm{MCT}$, or some higher volume fraction [97]) remains relevant for volume fractions well removed from the singularity and has a range of inf uence which extends back to dense equilibrium fl id states. More recently the MCT-ITT $S(\mathbf{k})$ has been evaluated numerically for two-dimensional hard-discs under shear at fi ite values of $P e$ [75], without invoking any 
additional isotropic approximations (see section 7.6). These calculations show only qualitative agreement with Brownian dynamics simulation results and overestimate the magnitude of the distortion by around an order of magnitude, a failing which is attributed to the fact that ITT-MCT apparently underestimates the speeding up of structural relaxations induced by the shear fow. This is to be contrasted with pair Smoluchowski-based approaches (e.g. [168, 182]) which underestimate the magnitude of the low shear distortion for dense fuid states $(\phi \sim 0.5)$ by around an order of magnitude. It therefore appears that neither the pair Smoluchowski nor the ITT-MCT approach can account adequately for the shear induced distortion of the microstructure.

\subsection{Applications}

Explicit numerical solution of the ITT-MCT constitutive equation (equations (68)-(70)) has been performed for a one-component system of hard-spheres under steady fows of various geometry [17]. However, the computational resources required to solve the anisotropic equations $(68)-$ (70) in three dimensions over many decades of time are daunting and the eff cient numerical algorithms required to reduce the computational load are still under development. Nevertheless, it is hoped that much of the essential physics may be captured by solving a simplif ed set of equations in which the advected wavevectors are approximated by an isotropic average $\mathbf{k}\left(t, t^{\prime}\right) \rightarrow k_{\mathrm{is}}\left(t, t^{\prime}\right)$, where

$$
k_{\mathrm{is}}^{2}\left(t, t^{\prime}\right)={ }_{4 \pi}^{1} \int \mathrm{d} \Omega k^{2}\left(t, t^{\prime}\right) .
$$

This technical approximation has been successfully applied to the case of simple shear $[57,195]$ and enables the angular integrals entering equations $(68)-(70)$ to be performed analytically. For two-dimensional systems algorithms have been developed which enable accurate numerical solution of the ITT-MCT equations without additional isotropic approximation [75].

In fgure 14 fow curves for hard-spheres resulting from solution of equations (68)-(70) subject to the isotropic approximation (77) are shown for both steady shear and steady planar extensional fow. For these two choices of fow the def ning velocity gradient tensors are given by

$$
\boldsymbol{\kappa}_{\mathrm{s}}=\left(\begin{array}{ccc}
0 & \dot{\gamma} & 0 \\
0 & 0 & 0 \\
0 & 0 & 0
\end{array}\right) \quad \boldsymbol{\kappa}_{\mathrm{e}}=\left(\begin{array}{ccc}
\dot{\gamma} & 0 & 0 \\
0 & -\dot{\gamma} & 0 \\
0 & 0 & 0
\end{array}\right)
$$

Specifica ly, f gure 14 shows $\sigma_{x y}$ under shear fow and $\Delta \sigma \equiv$ $\sigma_{\mathrm{xx}}-\sigma_{\mathrm{yy}}$ under planar extension for hard-spheres as a function of $P e$, for various volume fractions around the glass transition. The equilibrium structure factors used as input for these calculations were provided by the monodisperse PercusYevick theory [15]. For extensional fows it is natural to plot the stress difference $\Delta \sigma$ as a function of $P e$, as this is simply related to the extensional viscosity $\eta_{\mathrm{e}}=\Delta \sigma / \dot{\gamma}$. Flow curves below the glass transition show a regime of linear response, characterized by a constant viscosity, followed by shear thinning as $P e$ is increased. On approaching $\phi_{\mathrm{g}}$
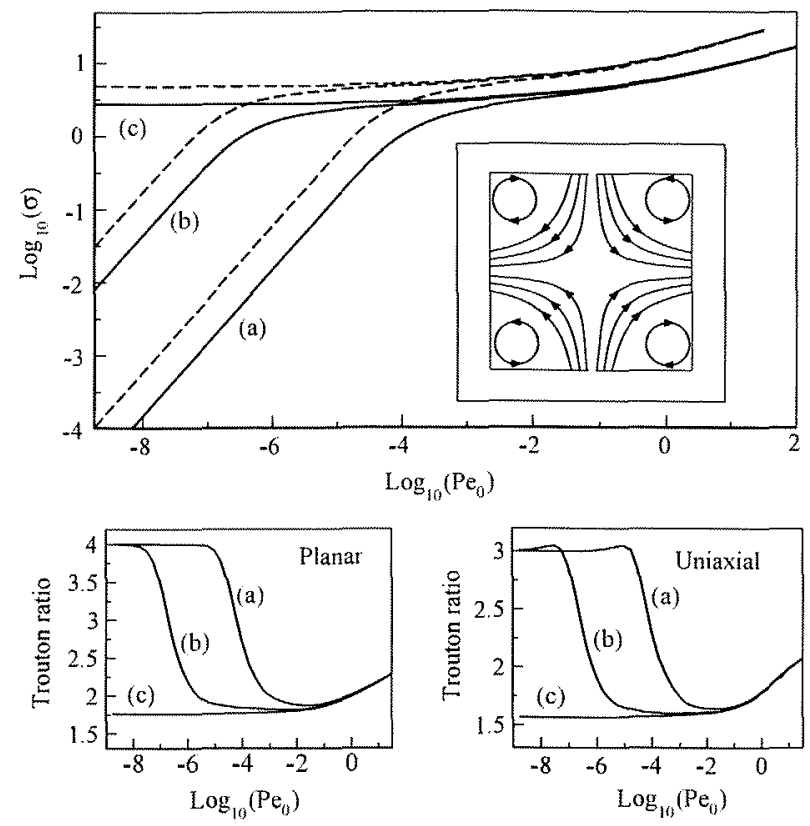

Figure 14. Flow curves from the microscopic ITT-MCT approach of [17] for hard-spheres at three different volume fractions close to the glass transition $\phi_{c}$. Full lines show the steady shear stress, $\sigma=\sigma_{x y}$ in units of $k_{\mathrm{B}} T /(2 R)^{3}$ under shear fl $\mathrm{w}$ as a function of $P e$ Broken lines show the stress difference $\sigma=\sigma_{x x}-\sigma_{y y}$ (related to the extensional viscosity) under planar extensional fow. Each curve is labelled according to the distance in volume fraction from the glass transition, $\Delta \phi=\phi-\phi_{c}$. (a) and (b) are fuid states with $\Delta \phi=-10^{-4}$ and $-10^{-3}$, respectively. State (c) is in the glass, $\Delta \phi=10^{-4}$, and exhibits a dynamic yield stress for both $\mathrm{f}$ ow geometries. The inset shows a possible realization of planar extensional fow. The lower two panels show the Trouton ratio $\sigma_{\mathrm{xx}}-\sigma_{\mathrm{yy}} / \sigma_{x y}$ as a function of $P e$ for both uniaxial and planar extensional fow.

from below the linear regime moves to lower values of $P e$ and disappears entirely on crossing the glass transition. The resulting plateau in the fow curves identifes a dynamical yield stress (see section 5.4 ) for both of the considered fow geometries.

In the lower panels of f gure 14 the Trouton ratio $\left(\sigma_{\mathrm{xx}}-\right.$ $\left.\sigma_{y y}\right) / \sigma_{x y}[29]$ is shown as a function of $P e$ for both planar and uniaxial extensional fow [17]. For fuid states in the linear regime Trouton's rules assert that the ratio of extensional to shear viscosity $\eta_{\mathrm{e}} / \eta_{\mathrm{s}}$ takes the values 4 and 3 for planar and uniaxial extension, respectively. These characteristic ratios arise from purely geometrical considerations and emerge naturally from the ITT-MCT approach as the $P e \rightarrow 0$ limiting values of the Trouton ratio. For glassy states (curves labelled (c) in f gure 14) the structural relaxation time diverges and the linear response regime vanishes. As a consequence, the classical Trouton ratios are not recovered for glassy states in the limit $\mathrm{Pe} \rightarrow 0$ and nontrivial values dictated by the dynamical yield stress may be identifed. The results for extensional fow presented in [17] alongside those for simple shear [ 135$]$ thus provide the frst steps towards the prediction from f rst-principles theory of a dynamic yield stress surface for glasses [210]. Calculation of the yield surface from a 
simplif ed schematic version of equations $(68)-(70)$ will be discussed in section 7.8 below.

Going beyond steady fow, in [02] experiments on PMMA colloidal dispersions, molecular dynamies simulation and the ITT-MCT approach of [16] were combined to study the evolution of stresses during start-up shear fow for high volume fraction fuids close to glassy arrest. The sudden onset of a steady shear $\mathrm{fl} \mathrm{w}$ leads to the build-up of stresses in the systems as a function of the accumulated strain $\gamma \equiv \dot{\gamma} t$. For small values of $\gamma$ the response is elastic (as described by equation (72)), whereas for large strains the system enters steady state viscous fow with a stress independent of $\gamma$. In between these two limits, typically at strains around $10 \%$, the stress exhibits a maximum as the local microstructure is broken up by the external fow [138]. Although a stress 'overshoot' in response to start-up shear fow is a rather generic feature of the rheology of complex fuids, its microscopic origins remain poorly understood. A central novel aspect of [62] was thus to connect the stress overshoot to anomalous behaviour in the mean-squared-displacement ('superdiffusion') identif ed in both simulation and confocal microscopy experiments. From the exact equation (49) it is clear that the only way in which the shear stress can exhibit a maximum is if the modulus under steady shear, $G_{s s}(t)$, becomes negative at long times. Figure 5 shows that $G_{\mathrm{ss}}(t)$ from the ITT-MCT approach (employing approximation (77)) indeed predicts negative values at long times. The inset to f gure 5 shows the same data as a function of strain and demonstrates that the negative region of $G_{s 5}(t)$ occurs at around $10 \%$ strain, consistent with the position of the stress overshoot.

\subsection{Schematic model}

The microscopic ITT-MCT constitutive equation outlined in section 7.4 provides a route to frst-principles prediction of the rheological behaviour of arrested colloidal states. However; the anisotropic, wavevector-dependent expressions are rather intractable for three-dimensional fows, hindering both their practical use and interpretation. In order to facilitate numerical calculations for fows of interest a simplifed 'schematic' version of the tensorial microscopic theory has very recently been proposed [18]. Such schematic models have proved invaluable in the analysis of mode-coupling theories and provide a simpler set of equations which retain the essential mathematical structure of the microscopic theory $[57,98]$.

Applying the isotropic approximation (77) to the microscopic ITT-MCT expression for the stress (66) enables the angular integrals to be performed explicitly, leading to the simplif ed form

$$
\sigma(t)=\int_{-\infty}^{t} \mathrm{~d} t^{\prime}\left[-{ }_{\partial t^{\prime}}^{\partial} B\left(t, t^{\prime}\right)\right] G\left(t, t^{\prime}\right),
$$

where $\mathbf{B}$ is the Finger tensor and an explicit expression for $G\left(t, t^{\prime}\right)$ may be found in [18]. By disregarding all wavevector dependence the modulus can be expressed in terms of a singlemode transient density correlator

$$
G\left(t, t^{\prime}\right)=v_{\alpha} \Phi^{2}\left(t, t^{\prime}\right)
$$

where $v_{\sigma}=G(t, t)$ is a parameter measuring the strength of stress fuctuations (typically taking values of the order $100 k_{\mathrm{B}} T / R^{3}$ for hard-sphere-like colloids). A schematic equation-of-motion for $\Phi\left(t, t^{\prime}\right)$ may be obtained by neglecting the wavevector dependence of the microscopic expression (68), leading to

$$
\dot{\Phi}\left(t, t^{\prime}\right)+\Gamma\left(\Phi\left(t, t^{\prime}\right)+\int_{t^{\prime}}^{t} \mathrm{~d} s m\left(t, s, t^{\prime}\right) \dot{\Phi}\left(s, t^{\prime}\right)\right)=0
$$

where the single decay rate $\Gamma$ simply sets the timescale and may thus be set to unity. Experience with the construction of schematic MCT models both in the quiescent [98] and steady shear cases [57] combined with analysis of the way in which strain enters the microscopic memory (69) lead to the following schematic ansatz for the memory function

$$
m\left(t, t^{\prime}, t_{0}\right)=h\left(t, t_{0}\right) h\left(t, t^{\prime}\right)\left[\nu_{1} \Phi\left(t, t^{\prime}\right)+\nu_{2} \Phi^{2}\left(t, t^{\prime}\right)\right] .
$$

The parameters $\nu_{1}$ and $\nu_{2}$ represent in an unspecifie way the role of $S_{\mathrm{q}}$ in the microscopic theory and, following standard MCT practice, are given by $v_{2}=2$ and $v_{1}=2(\sqrt{2}-1)+$ $\epsilon /(\sqrt{ } 2-1)$. The separation parameter $\epsilon$ encodes the distance from the glass transition, with negative values corresponding to $f$ uid states and positive values corresponding to glass states. Finally, the $h$-function is given by

$$
h\left(t, t_{0}\right)=\begin{gathered}
\gamma_{c}^{2} \\
\gamma_{c}^{2}+v\left(I_{1}\left(t, t_{0}\right)-3\right)+(1-v)\left(I_{2}\left(t, t_{0}\right)-3\right)
\end{gathered},
$$

where $\gamma_{c}$ sets the strain scale (typically $\gamma_{c} \approx 10 \%$ ) and $v$ is a mixing parameter $(0<v<1)$. The invariants of the Finger tensor, $I_{1}=\operatorname{Tr} \mathbf{B}$ and $I_{2}=\operatorname{Tr} \mathbf{B}^{-1}$ incorporate the fuidizing infuence of fow into the memory function. Requiring that fow enter via the Finger tensor alone guarantees that the resulting schematic theory is material objective (see section 2.3), consistent with the fully microscopic theory.

The schematic expression for the stress (79) is closely related to the Lodge equation of continuum mechanics (see section 2.1 ). Integrating equation (79) by parts yields

$$
\sigma(t)=\int_{-\infty}^{t} \mathrm{~d} t^{\prime} B\left(t, t^{\prime}\right)\left(\begin{array}{c}
\partial \\
\partial t^{\prime}
\end{array} \Phi^{2}\left(t, t^{\prime}\right)\right) .
$$

Comparison of this expression with equation (5) shows that the present schematic theory goes considerably beyond the standard Lodge equation by incorporating memory which is both nonexponential and a function of two time arguments, ref ecting the loss of time-translational invariance under timedependent fow. Replacing the correlator with a simple exponential trivially recovers the Lodge equation (5).

The tensorial schematic theory given by equations (79)(83) has been applied to predict fow curves under shear and extensional fow, normal stresses under shear and the transient stress response to step strain. In all cases tested so far the predictions of the schematic model are in good qualitative agreement with those available from the microscopic theory using the isotropic approximation (77) in three dimensions $[16,17,57,135]$ and exact numerical solution in two dimensions [75]. The schematic theory predicts a 

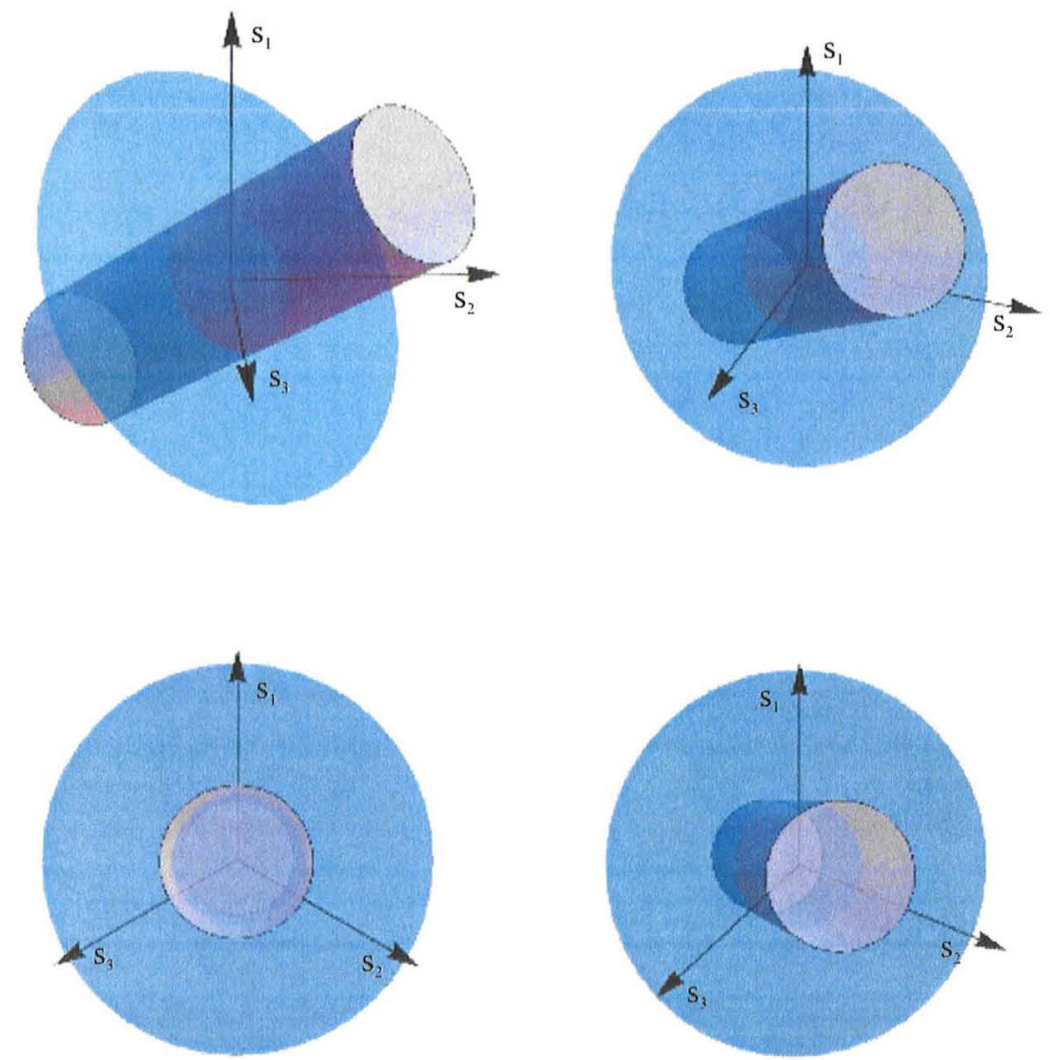

Figure 15. The state of stress of a material under applied force can be represented by a point in the three-dimensional space of principal stresses. The cylinder shown here is the surface define by the von Mises criterion (86). Stress states lying within the cylinder are deformed by the applied stress, but do not yield, whereas states outside the cylinder exhibit plastic fl w. Due to invariance along the 'hydrostatic' axis $s_{1}=s_{2}=s_{3}$ (arising from incompressibility) the yield surface is usually viewed in the 'deviatoric' plane perpendicular to this (the lower left representation in this f gure).

positive value for the frst normal stress difference $N_{1}=$ $\sigma_{\mathrm{xx}}-\sigma_{\mathrm{yy}}$ under shear $\mathrm{fl} \mathrm{w}$ in accord with microscopic ITTMCT calculations. On the other hand, the second normal stress difference $N_{2}=\sigma_{\mathrm{yy}}-\sigma_{\mathrm{zz}}$ is from equation (79) identically zero, in disagreement with both analytical low $P e$ analysis of the pair Smoluchowski equation [154] and colloidal experiments [207] (f nite negative values are found). The disappearance of $N_{2}$ from the schematic theory can be traced back to the isotropic approximation leading to equation (79) which effectively kills off this feature of the fully microscopic theory.

\subsection{Yield stress surface}

A striking feature of the theory developed in [18] is that it permits direct calculation of a dynamic yield stress surface for glasses. Related static yield surfaces have been empirically postulated and employed for over a century in the engineering community to study the yielding of amorphous solids (see also section 5.4). The two classical criteria for determining the onset of plastic yield are due to Tresca [208] and von Mises [209]. The Tresca criterion asserts that a material will yield when the maximum shear stress due to the deformation exceeds a critical value. Recalling that an external force imposed on a material can be represented as a stress tensor which can be diagonalized to obtain values for the three principal stresses $s_{1}, s_{2}$ and $s_{3}$, the Tresca criterion can be compactly stated in the form [210]

$$
\operatorname{Max}\left(\left|s_{1}-s_{2}\right|,\left|s_{2}-s_{3}\right|,\left|s_{1}-s_{3}\right|\right)=\sqrt{ } 3 \sigma_{s s}^{y},
$$

where $\sigma_{s s}^{y}$ is the shear stress at yield under a simple shear deformation. According to equation (85) knowledge of $\sigma_{s s}^{y}$ is thus suff cient to determine the mechanical stability of a material under an arbitrary applied force. Alternatively, the von Mises criterion requires that the distortion strain energy exceeds a critical value at yield [210]

$$
{ }_{6}^{1}\left(\left(s_{1}-s_{2}\right)^{2}+\left(s_{2}-s_{3}\right)^{2}+\left(s_{1}-s_{3}\right)^{2}\right)=\left(\sigma_{s s}^{y}\right)^{2} .
$$

Both the Tresca and von Mises criteria have proven to be in reasonable qualitative agreement with yield experiments on crystalline metals ${ }^{11}$. Two main assumptions underly equations (85) and (86): (i) the microscopic rearrangements leading to plastic deformation do not lead to signif cant dilation of the material, (ii) that residual stresses arising from the deformation history of the sample do not inf uence the yielding (i.e. there is no Bauschinger effect).

11 There is a useful short discussion of the von Mises, Tresca and generalized pressure dependent yield criteria in metals in section 4.2.3.3 of [211]. 

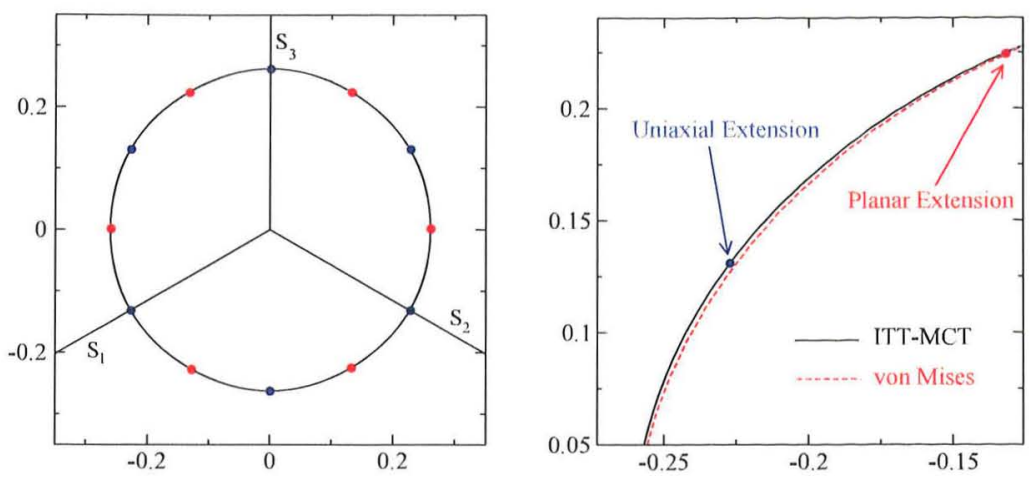

Figure 16. Left panel: the dynamical yield surface from ITT-MCT [18] for a glass with $\epsilon \approx 3\left(\phi-\phi_{\mathrm{gt}}\right)=10^{-3}$, where $\phi_{\mathrm{gt}}$ is the volume fraction at the hard-sphere glass transition, in the space of principal stresses $\left(s_{1}, s_{2}, s_{3}\right)$ as viewed along the hydrostatic axis $s_{1}=s_{2}=s_{3}$ (stress in units of $\left.k_{\mathrm{B}} T /(2 R)^{3}\right)$. The red points correspond to planar extensional fow and the blue points to uniaxial extensional $\mathrm{f}$ ow. Right panel: a closer view reveals that the surface is not a perfectly circular cylinder (as predicted by the von Mises criterion for static yielding) and that maximal deviation from circularity occurs at points of pure uniaxial extension. These deviations are connected with the existence of fin te normal stress differences.

It is useful to interpret equation (86) geometrically in the space of principal stresses, where it describes a surface separating elastically deformed states from states of plastic fow. Equation (86) defi es a circular cylinder with axis along the line $s_{1}=s_{2}=s_{3}$ and radius $\sqrt{ } 2 \sigma_{s s}^{y}$. The symmetry about this 'hydrostatic' axis is a geometrical ref ection of the fact that the yield condition (86) is independent of hydrostatic pressure. The plane which passes through the origin and which lies perpendicular to the cylinder axis is the socalled deviatoric plane. All yield stress surfaces which are independent of hydrostatic pressure may be projected without loss of information onto the deviatoric plane. In the case of the von Mises and Tresca criteria this generates a circle (see f gure 15) and a hexagon, respectively.

The results presented in [18] reveal intriguing connections between the static yielding discussed above and dynamic yield, as determined from the $P e \rightarrow 0$ values of the fow curves. Within the ITT-MCT approach, for any given steady fow fie d (e.g. shear, uniaxial extension) there exists for glassy states a f nite stress tensor in the limit of vanishing fow rate. This stress tensor at yield may be diagonalized to obtain three eigenvalues and plotted as a point in the Cartesian space of principal stresses. By considering all possible nondegenerate fl ws (made possible by a suitable parameterization of the velocity gradient tensor [18]) a closed locus of points may be constructed in the space: the dynamic yield stress surface. In [18] this procedure was realized using the schematic model equations (79)-(83).

In f gure 16 we show the deviatoric projection of the dynamical yield stress surface of a colloidal glass, as predicted by the schematic model. To a fist approximation the numerically calculated dynamical yield surface from the theory agrees well with the empirical von Mises criterion for static yield (86). However, closer inspection (see f gure 16, right panel) reveals that deviations at around the per cent level occur, with the maximal deviation located at points of pure uniaxial extension. A careful analysis of the schematic equations reveals that this f ne structure of the surface can be attributed to the existence of a f nite f rst normal stress difference [18].
Expanding the schematic model result to frst order in $N_{\mathrm{i}}^{y}$, the frst normal stress difference at yield, provides an explicit expression for the schematic model yield surface [18]

$$
\begin{gathered}
{ }_{6}^{1}\left(\left(s_{1}-s_{2}\right)^{2}+\left(s_{2}-s_{3}\right)^{2}+\left(s_{1}-s_{3}\right)^{2}\right)=\left(\sigma_{\mathrm{ss}}^{y}\right)^{2} \\
+{ }_{12}^{1}\left(N_{1}^{y}\right)^{2}+\begin{array}{c}
3\left(1-A^{2}\right) \\
\left(3+A^{2}\right)^{3 / 2}
\end{array} N_{1}^{y} \sigma_{\mathrm{ss}}^{y},
\end{gathered}
$$

where $0<A<1$ parameterizes the geometry of the imposed fow. Equation (87) thus describes a noncircular cylinder with a radius which varies according to the value of the parameter $A$, each value of which corresponds to a given azimuthal angle about the hydrostatic axis. Higher order terms in the expansion (87) exist (and can be explicitly calculated) but remain numerically negligible due to the smallness of $N_{1} / \sigma_{\mathrm{ss}}^{y}$.

Very recent experiments on yielding soft materials have attempted to determine the shape of the yield surface [212]. The novel rheometer employed in [212] applies a combined squeeze and rotational shear $\mathrm{fl} \mathrm{W}$ to a material sample loaded between two parallel plates. The claim is that by independently varying the rotation and squeeze rate it may become possible to explore the entire family of f ows in a way analogous to the mathematical parameterization of the velocity gradient tensor employed in [18] to calculate the schematic model yield surface. However, it remains uncertain whether the superposition of two shear forvs (radial Poiseuille and tangential homogeneous shear) is really suff cient to map the yield surface. Figure 17 shows experimental data taken using three different yielding materials: a Carbopol gel and two different emulsions [212].

The yield data are shown in the rotational shear stress $\left(\tau_{\mathrm{r} \theta}\right)$, squeeze shear stress $\left(\tau_{\mathrm{rz}}\right)$ plane. While the data shown in f gure 17 are not inconsistent with the von Mises criteria, it may well be that the chosen superposition fow actually constrains the surface to be spherical, regardless of the true form of the yield surface. It would certainly be remarkable if the soft materials considered in [212] obey yield criteria developed for crystalline solids, despite the very different underlying microscopic plasticity mechanisms [213]. 


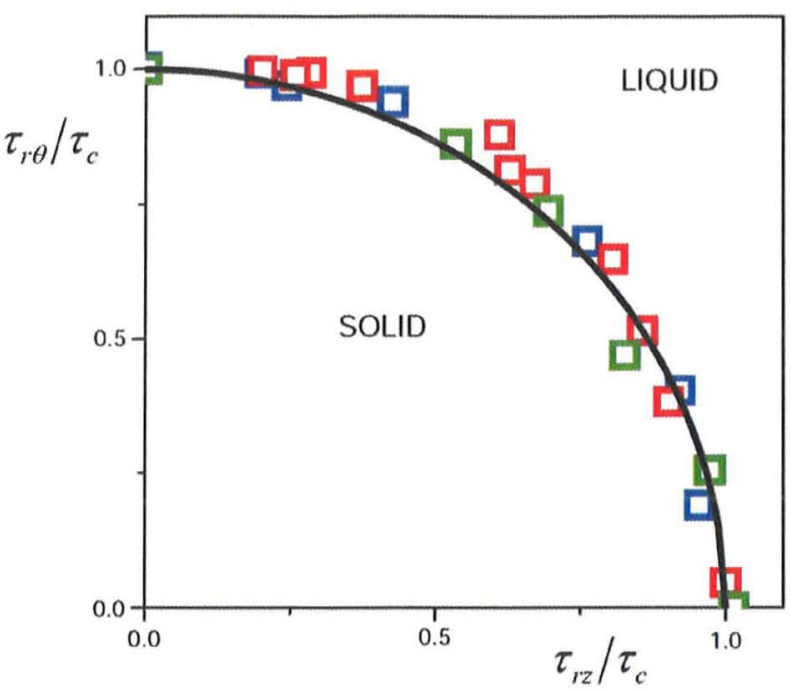

Figure 17. The yield stress surface in the $\tau_{\mathrm{r} \theta}$ (rotational shear stress), $\tau_{\mathrm{rz}}$ (squeeze shear stress) plane, where the stresses have been scaled by the yield stress $\tau_{c}$ in simple shear. The blue and red squares show results for two different emulsions with differing values of $\tau_{c}$ (28 and $52 \mathrm{~Pa}$ ). The green squares are data taken using a carbopol gel $\left(\tau_{c}=70 \mathrm{~Pa}\right)$. In this representation the von Mises criterion (86) becomes a circle and is indicated by the solid line. Reprinted by permission from MacMillan Publishers Ltd: Nat. Mater. 9115 , copyright (2010)

Whether the microscopic theory (equations (68)-(70)) indeed predicts a similar dynamic yield surface, as expected, and its relationship to static yielding in glassy materials remain important open problems. Nevertheless, the results from the schematic model are promising and represent a considerable step towards a microscopic derivation of material specifi yield surfaces from f rst-principles.

\section{Outlook}

In this review we have attempted to provide an overview of the rheological phenomenology presented by colloidal dispersions and to outline some of the leading theoretical approaches aiming to rationalize this. It is clear that much remains to be done and that existing theories have met with only partial success in solving the complex many-body problem of driven, strongly interacting colloidal systems. We hope that both the presentation and choice of topics contained within the present work serve to emphasize the common ground between different theories (continuum mechanics, pair Smoluchowski treatments and Green-Kubo based approaches), as well as to highlight where progress still needs to be made.

One of the clear def ciencies of approximate closures of the pair Smoluchowski equation is their apparent inability to describe, even qualitatively, the slow structural relaxation time present in colloidal dispersions at $\mathrm{f}$ nite volume fraction. This failing is inherent in the Markovian character of the approximate closures, which follows as a natural consequence of applying equilibrium statistical mechanical relations to connect pair and triplet correlation functions. A clear challenge to future theories which attempt to improve this situation is thus to tackle directly the intrinsic difference between nonequilibrium and equilibrium by confronting the irreducible term (see equation (46)) containing the missing physics. Moreover, the existing numerical data in the literature are restricted, largely for technical reasons, to the low $\mathrm{Pe}$ limit. While this enables interesting analysis of the zero-shear viscosity and leading order microstructural distortion, there is an absence of data for the nonlinear rheology as a function of Pe.

Despite the success of the ITT-MCT approach in describing the nonlinear response of states close to dynamic arrest, there remain gaps in the theoretical formulation which should be flled and many fundamental questions are yet to be addressed. A notable omission is that the present formulation of the theory does not enable incorporation of hydrodynamic interactions. The inf uence of hydrodynamics on the rheology of densely packed glassy states is largely unexplored and their incorporation into the theory, even at a crude approximate level, would therefore be of considerable interest. Moreover, due to the recent nature of the ITTMCT theory and the complexity of numerically solving the equations, many fows of rheological interest remain to be explored in detail. Of particular importance is to assess the predictions of the theory for nonsteady fows where interesting interaction effects between yielding and the time-dependent strain feld may be envisaged (e.g. large amplitude oscillatory shear, where higher harmonics will contribute to the stress response)

\section{Acknowledgments}

I would particularly like to thank $M$ Fuchs, M E Cates, Th Voigtmann and M Krüger for many stimulating discussions. Support was provided by the SFB TR6 and the Swiss National Science Foundation.

\section{References}

[1] Mezzenga R, Schurtenberger P, Burbidge A and Michel M 2005 Nat. Mater. 4729

[2] Larson R G 1999 The Structure and Rheology of Complex Fluids (New York: Oxford University Press)

[3] Brown E and Jaeger H J 2009 Phys. Rev. Lett. 103086001

[4] Fall A, Huang N, Bertrand F, Ovarlez G and Bonn D 2008 Phys. Rev. Lett. 100018301

[5] Coussot P 2005 Rheometry of Pastes, Suspensions and Granular Materials (New York: Wiley)

[6] Russel W B, Saville D A and Schowalter W R 1989 Colloidal Dispersions (Cambridge: Cambridge University Press)

[7] van Megen W and Pusey P 1991 Phys. Rev. A 435429

[8] van Megen W and Underwood S 1993 Phys. Rev. Lett. 702766

[9] van Megen W and Underwood S 1994 Phys. Rev. E 494206

[10] Götze W and Sjögren L 1992 Rep. Prog. Phys. 55241

[11] Götze W 1999 J. Phys.: Condens. Matter 11 Al

[12] Aarts D G A L et al 2004 Science 304847

[13] Mandelstam L 1913 Ann. Phys. 41609

[14] Dhont J K G 1996 An Introduction to the Dynamics of Colloids (Amsterdam: Elsevier)

[15] Hansen J-P and McDonald I R 1986 Theory of Simple Liquids (London: Academic)

[16] Brader J M, Voigtmann Th, Cates M E and Fuchs M 2007 Phys. Rev. Lett. 98058301 
[17] Brader J M, Cates M E and Fuchs M 2008 Phys. Rev. Lett. 101138301

[18] Brader J M et al 2009 Proc. Natl Acad. Sci. USA 10615180

[19] Fuchs M 2009 High Dolid Dispersions ed M Cloitre (Berlin Springer)

[20] Fuchs M and Cates M E 2009 J. Rheol. 53957

[21] Doi M and Edwards S F 1989 The Theory of Polymer Dynamics (Oxford: Oxford University Press)

[22] Larson R G 1988 Constitutive Equations for Polymer Melts and Solutions (Boston, MA: Butterworth)

[23] Macleish T C B 2002 Adv. Phys. 511379

[24] Cates M E and Fielding S M 2006 Adv. Phys. 55799

[25] Coussot P 2007 Soft Matter 3528

[26] Vermant J and Solomon M J 2005 J. Phys.: Condens. Matter 17 R187

[27] Lionberger R A and Russel W B 2000 Adv. Chem. Phys. 111399

[28] Morris J F 2009 Rheol. Acta 48827

[29] Bird R B, Armstrong R C and Hassager O 1987 Dynamics of Polymeric Liquids (New York: Wiley)

[30] Batchelor G K 1967 An Introduction to Fluid Dynamics (Cambridge: Cambridge University Press)

[31] Truesdell C and Noll W 1965 The Nonlinear Field Theories of Mechanics (Berlin: Springer)

[32] de Gennes P G 1983 Physica A 11843

[33] Ryskin G 1985 Phys. Rev. A 32 1239

[34] Sollich P, Lequeux F, Hebraud P and Cates ME 1997 Phys. Rev. Lett. 782020

[35] Sollich P 1998 Phys. Rev. E 58738

[36] Fielding S M, Sollich P and Cates M E 2000 J. Rheol. 44323

[37] Crocker J and Grier D G 1996 J. Colloid Interface Sci. 179298

[38] Prasad V, Semwogerere D and Weeks E R 2007 J. Phys.: Condens. Matter 19113102

[39] Besseling R, Isa L, Weeks E R and Poon W C K 2009 Adv. Colloid Interface Sci. 146 I

[40] Varnik F 2006 J. Chem. Phys. 125 ] 64514

[41] Sierou A and Brady J F 2001 J. Fluid Mech. 448115

[42] Doliwa B and Heuer A 2000 Phys. Rev. E 616898

[43] Berthier L, Barrat J-L and Kurchan J 2000 Phys. Rev. E 615464

[44] Berthier L and Barrat J-L 2002 J. Chem. Phys. 1166228

[45] Bouchaud J-P, Cugliandolo F, Kurchan J and Mézard M 1998 Spin Glasses and Random Fields ed A P Young (Singapore: World Scientif c)

[46] Riskin H 1989 The Fokker-Planck Equation (Berlin Springer)

[47] Kim S and Karilla S J 1991 Microhydrodynamics, Principles and Selected Applications (Boston, MA: Butterworth-Heinemann)

[48] Jorquera H and Dahler J S 1994 J. Chem. Phys. 1011392

[49] Brady J F and Bossis G 1988 Annu. Rev. Fluid Mech. 20111

[50] Banchio A J and Brady J F 2003 J. Chem. Phys. 11810323

[51] Brady J F 2001 Chem. Eng. Sci. 562921

[52] Sami S 1997 Stokesian dynamics simulation of extensional fl w of Brownian suspensions Masters Thesis California Institute of Technology

[53] Mathews J and Walker R L 1970 Mathematical Methods of Physics (Reading, MA: Addison-Wesley)

[54] Foister R T and van de Ven T G M 1980 J. Fluid Mech. 96105

[55] Taylor G I 1953 Proc. R. Soc. A 219186

[56] Brady J F and Morris J F 1997 J. Fluid Mech. 348103

[57] Fuchs M and Cates M E 2003 Faraday Discuss. 123267

[58] Bender J W and Wagner N J 1996 J. Rheol. 40899

[59] Ballesta P, Besseling R, Isa L, Petekidis $G$ and Poon W C K 2008 Phys. Rev. Lett. 101258301

[60] Crassous J J et al 2008 J. Chem. Phys. 128204902

[61] Siebenbürger M, Fuchs M, Winter H and Ballauff M 2009 J. Rheol. 53707

[62] Zausch J et al 2008 J. Phys.: Condens. Matter 20404210
[63] Rauscher M, Dominguez A, Krüger M and Penna F 2007 J. Chem. Phys. 127034905

[64] Alder B J and Wainwright T E 1957 J. Chem. Phys. 271208 Hoover W G and Ree F H 1968 J. Chem. Phys. 493609

[65] Gasser U 2009 J. Phys.: Condens. Matter 21203101

[66] Voigtmann Th, Puertas A M and Fuchs M 2004 Phys. Rev. E 70061506

[67] Cates ME and Ramaswamy S 2006 Phys. Rev. Lett. 96135701

[68] Chong S-H 2008 Phys. Rev. E 78041501

[69] Berthier L et al 2006 Science 3105755

[70] Biroli G, Bouchaud J-P, Miyazaki K and Reichmann D R 2006 Phys. Rev. Lett. 97195701

[71] Duri A, Bissig H, Trappe V and Cipelletti L 2005 Phys. Rev. E 72051401

[72] Cerbino R and Trappe V 2008 Phys. Rev. Lett. 100188102

[73] Weeks E R, Crocker J C, Levitt A C, Schofie d A and Weitz D A 2000 Science 287627

[74] Bayer M et al 2007 Phys. Rev. E 76011508

[75] Henrich O, Weyßer F, Cates M E and Fuchs M 2009 Phil. Trans. R. Soc. A 3675033

[76] Götze W and Voigtmann Th 2003 Phys. Rev. E 67021507

[77] Hajnal D, Brader J M and Schilling R 2009 Phys. Rev. E 80021503

[78] Trappe V and Sandkuhler P 2004 Curr. Opin. Colloid Interface Sci. 8494

[79] Segrè P N et al 2001 Phys. Rev. Lett. 866042

[80] Pham K N J et al 2002 Science 296104

[81] Pham K N J et al 2004 Phys. Rev. E 69011503

[82] Dijkstra M, Brader J M and Evans R 1999 J. Phys.: Condens. Matter 1110079

[83] Brader J M and Evans R 2002 Physica A 306287

[84] Laurati M et al 2009 J. Chem. Phys. 130134907

[85] Dibble C J, Kogan M and Solomon M J 2006 Phys. Rev. E 74041403

[86] Manley S et al 2005 Phys. Rev. Lett. 94238302

[87] Foff G, De Michele C, Sciortino F and Tartaglia P 2005 Phys. Rev. Lett. 94078301

[88] Sciortino F, Tartaglia P and Zaccarelli E 2005 J. Phys. Chem. B 10921942

[89] Archer A J and Wilding N B 2007 Phys. Rev. E 760315011

[90] Klix C L, Royall C P and Tanaka H 2010 Phys. Rev. Lett. 104165702

[91] Bianchi E, Largo J, Tartaglia P, Zaccarelli E and Sciortino F 2007 Phys. Rev. Lett. 97168301

[92] Zaccarelli E 2007 J. Phys.: Condens. Matter $1932310 \mathrm{l}$

[93] Pham K N et al 2006 Europhys. Lett. 75624

[94] Laun H M 1984 Angew. Makromol. Chem. 123335

[95] Lionberger R A and Russel W B 1994 J. Rheol. 381885

[96] Nägele G and Bergenholtz J 1998 J. Chem. Phys. 1089893

[97] Cheng Z et al 2002 Phys. Rev. E 65041405

[98] Götze W 1991 Liquids, Freezing and the Glass Transition ed J-P Hansen, D Levesque and J Zinn-Justin (Amsterdam: North-Holland)

[99] Phung T and Brady J F 1992 Slow Dynamics in Condensed Matter vol 256, ed K Kawasaki, M Tokuyama and T Kawakatsu (Woodbury, NY: AIP) p 391

[100] Rastogi S R and Wagner N J 1995 Comput. Chem. Eng. 19693

[101] Bossis G and Brady JF 1984 J. Chem. Phys. 805141

[102] Melrose J R and Ball R C 2004 J. Rheol. 48937

[103] Melrose J R and Ball R C 2004 J. Rheol. 48961

[104] Kulkarni S D and Morris J F 2009 J. Rheol. 53417

[105] Mewis J and Wagner N J 2009 Adv. Colloid Interface Sci. $147-148214$

[106] D'Haene P, Mewis J and Fuller G G 1993 J. Colloid. Interface Sci. 156350

[107] Barnes H A 1989 J. Rheol. 33329

[108] Hoffman R L 1972 Trans. Soc. Rheol. 16155

[109] Boersma W H, Laven J and Stein H N 1990 AlChE J. 36321

[110] Laun H M et al 1992 J. Rheol. 36743 
[111] Laun H M, Bung R and Schmidt F 1991 J. Rheol. 35999

[112] Bossis G and Brady JF 1989 J. Chem. Phys. 911860

[113] Kalman D P and Wagner N J 2009 Rheol. Acta 49897

[114] Hoffman R L 1998 J. Rheol. 42111

[115] Egres R G and Wagner N J 2005 J. Rheol. 49719

[116] Laun H 1994 J. Non-Newtonian Fluid Mech. 5487

[117] Frith W J, d'Heane P, Buscall R and Mewis J 1996 J. Rheol. 40531

[118] Cates M E, Holmes C B, Fuchs M and Henrich O 2004 Unifying Concepts in Granular Media and Glasses ed A Coniglio et al (Amsterdam: Elsevier)

[119] Holmes C B, Cates M E, Fuchs M and Sollich P 2005 J. Rheol. 49237

[120] Cates M E, Haw M D and Holmes C B 2005 J. Phys.: Condens. Matter $17 \$ 2517$

[121] Delhommelle J and Petravic J 2005 J. Chem. Phys. 123074707

[122] Mehta A, Barker G and Luck J M 2009 Phys. Today 62 (5) 40

[123] Gopalakrishnan V and Zukoski C F 2004 J. Rheol. $48132 !$

[124] Negi A S and Osuji C O 2009 Rheol. Acta 48871

[125] Poon W 2004 Science 304830

[126] Evans D J and Morriss G P 1986 Phys. Rev. Lett. 562172

[127] Evans D J et al 1992 Phys. Rev. A 466731

[128] Matin M L, Daivis P J and Todd B D 2000 J. Chem. Phys. 1139122

[129] Daivis P J, Matin M L and Todd B D 2003 J. Non-Newton. Fluid Mech. 111

[130] Lootens D, van Damme H, Hémar Y and Hébraud P 2005 Phys. Rev. Lett. 95268302

[131] Hébraud P 2009 Rheol. Acta 48845

[132] Foss D R and Brady J F 2000 J. Rheol. 44629

[133] Bergenholtz J, Brady J F and Vicic M 2002 J. Fluid Mech. 456239

[134] Zackrisson M, Stradner A, Schurtenberger P and Bergenholtz J 2006 Phys. Rev. E 73011408

[135] Fuchs M and Cates ME 2002 Phys. Rev. Lett. 89248304

[136] Besseling R, Weeks E R, Schofie d A B and Poon W C K 2007 Phys. Rev. Lett. 99028301

[137] Ovarlez G, Rodts S, Chateau X and Coussot P 2009 Rheol. Acta 48831

[138] Varnik F, Bocquet L and Barrat J-L 2004 J. Chem. Phys. 1202788

[139] Varnik F, Bocquet L, Barrat J-L and Berthier L 2003 Phys. Rev. Lett. 90095702

[140] Wagner N J and Russel W B 1989 Physica A $155475^{\circ}$

[141] Russel W B and Gast A P 1985 J. Chem. Phys. 841815

[142] Wagner N J 1994 J. Colloid Interface Sci. 161169

[143] Kim S and Miffl n R T 1985 Phys. Fluids 282033

[144] Medina-Noyola M 1988 Phys. Rev. Lett. 602705

[145] Brady J F 1993 J. Chem. Phys. 99567

[146] Attard P 1989 J. Chem. Phys. 913072

[147] Brader J M 2008 J. Chem. Phys. 128104503

[148] Kirkwood J G, Buff F P and Green M S 1949 J. Chem. Phys. 17988

[149] Yurkovetsky Y and Morris J F 2006 J. Chem. Phys. 124204908

[150] Einstein A 1906 Ann. Phys. 324289 see also Einstein A 1911 Ann. Phys. 339591 (erratum)

[151] Batchelor G K and Green J T 1972 J. Fluid Mech. $\$ 6375$

[152] Batchelor G K and Green J T 1972 J. Fluid Mech. 56401

[153] Batchelor G K 1977 J. Fluid Mech. 8397

[154] Brady J F and Vicic M 1995 J. Rheol. 39545

[155] Gadala-Maria F and Acrivos A 1980 J. Rheol. 24799

[156] Dhont J K G 1989 J. Fluid Mech. 204421

[157] Lionberger R A 1998 J. Rheol. 42843

[158] Ramaswamy S 1997 Theoretical Challenges in the Dynamics of Colloidal Fluids ed T McLeish (Dordrecht: Kluwer)

[159] Bławzdziewicz J and Szamel G 1993 Phys. Rev. E 484632

[160] Brady J F 1994 J. Fluid Mech. 272109

[161] Born M and Green M S 1949 A General Kinetic Theory of Liquids (Cambridge: Cambridge University Press)

[162] Kirkwood J G 1935 J. Chem. Phys. 3 300
[163] Rice S and Lekner J 1965 J. Chem. Phys. 423559

[164] Ontsuki T 1981 Physica A 108 A 441

[165] Ohtsuki T 1982 Physica A 110A 606

[166] Attard P and Stell G 1992 Chem. Phys. Lett. 189128

[167] Cichocki B 1987 Z. Phys. B $66 \$ 37$

[168] Szamel G 2001 J. Chem. Phys. 1148708

[169] Verlet L and Weis J-J 1972 Phys. Rev. A 5939

[170] Stell G 1964 Equilibrium Theory of Classical Fluids ed H L Frisch and JL Lebowitz (New York: Benjamin)

[171] Brader J M 2006 Int. J. Thermophys. 27394

[172] Verlet L 1964 Physica 3095

[173] Levesque D and Verlet L 1964 Phys. Lett. 1136

[174] Verlet L 1965 Physica 31959

[175] Verlet L 1966 Physica 32304

[176] Verlet L and Levesque D 1967 Physica 25.4254

[177] Wertheim M S 1967 J. Math. Phys. 8927

[178] Baxter R J 1968 Ann. Phys. 46509

[179] Stell G 1969 Mol. Phys. 16209

[180] Barrat J-L, Hansen J-P and Pastore G 1987 Phys. Rev. Lett. 582075

[181] Scherwinski K 1990 Mol. Phys. 70797

[182] Lionberger R A and Russel W B 1997 J. Chem. Phys. 106402

[183] Rogers F J and Young D A 1984 Phys. Rev. A 30999

[184] Lionberger R A and Russel W B 1997 J. Rheol. 41399

[185] Henrich O, Pfeifroth $O$ and Fuchs M 2007 J. Phys.: Condens. Matter 19205132

[186] Resibois P and Lebowitz J L 1975 J. Stat. Phys. 12483

[187] Ronis D 1984 Phys. Rev. A 291453

[188] Schwarzl JF and Hess S 1986 Phys. Rev. A 334277

[189] Wagner N J 2001 J. Non-Newton. Fluid Mech. 96177

[190] Ötinger H C 2005 Beyond Equilibrium Thermodynamics (Hoboken: Wiley)

[191] Evans D J and Morriss G 2008 Statistical Mechanics of Nonequilibrium Liquids (Cambridge: Cambridge University Press)

[192] Indrani A V and Ramaswamy S 1995 Phys. Rev. E 526492

[193] Miyazaki K and Reichman D R 2002 Phys. Rev. E 6605050

[194] Miyazaki K, Reichman D R and Yamamoto R 2004 Phys. Rev. E 70011501

[195] Miyazaki K, Wyss H M, Weitz D A and Reichmann D R 2006 Europhys. Lett. 75915

[196] Koblev V and Schweizer K S 2005 Phys. Rev. E 71021401

[197] Saltzman E J, Yatsenko G and Schweizer K S 2008 J. Phys. Condens. Matter 20244129

[198] Petekidis G, Vlassopoulos D and Pusey P N 2004 J. Phys.: Condens. Matter $16, \$ 3955$

[199] Fuchs M and Cates M E 2003 J. Phys.: Condens. Matter $15 S 401$

[200] Fuchs M and Ballauff M 2005 J. Chem. Phys. 122094707

[20I] Crassous J J et al 2006 J. Chem. Phys. 125204906

[202] Fuchs M and Cates M E 2005 J. Phys.: Condens. Matter $17 \$ 1681$

[203] van Kampen N G 1981 Stochastic Processes in Physics and Chemistry (Amsterdam: North-Holland) chapter XV Section 3

[204] McQuarrie D A 2000 Statistical Mechanics (Mill Valley, CA University Science Books)

[205] Cates M E 2003 Ann. Henri Poincaré 4 (Suppl, 2) S64)

[206] Fredrickson G H and Larson R G 1987 J. Chem. Phys. 861553

[207] Frank M, Anderson D, Weeks E R and Morris J F 2003 J. Fluid Mech. 493363

[208] Tresca H 1854 C. R. Acad. Sci. Paris 59754

[209] von Mises R 1913 Göttinger Nachrichten, Math._Phys. Klasse 582

[210] Hill R 1971 The Mathematical Theory of Plasticity (Oxford: Oxford University Press)

[211] Schuh C A, Hufnagel T C and Ramamurty U 2007 Acta Mater 554067

[212] Ovarlez G, Barrel Q and Coussot P 2010 Nat. Mater. 9115

[213] Schall P, Weitz D A and Spaepen F 2007 Science 3181895 